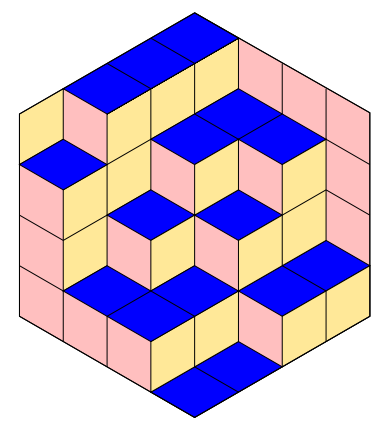

ALGEBRAIC COMBINATORICS

Grégory Chatel, Vincent Pilaud \& Viviane Pons

The weak order on integer posets

Volume 2, issue 1 (2019), p. 1-48.

<http://alco.centre-mersenne.org/item/ALCO_2019__2_1_1_0>

(C) The journal and the authors, 2019.

Some rights reserved.

(c) BY This article is licensed under the

Creative Commons ATtribution 4.0 InTERnational License.

http://creativecommons.org/licenses/by/4.0/

Access to articles published by the journal Algebraic Combinatorics on the website http://alco.centre-mersenne.org/ implies agreement with the Terms of Use (http://alco.centre-mersenne.org/legal/).

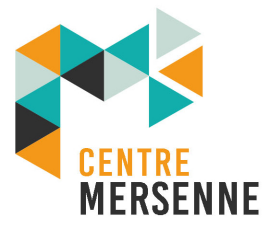

Algebraic Combinatorics is member of the Centre Mersenne for Open Scientific Publishing www.centre-mersenne.org 


\title{
The weak order on integer posets
}

\author{
Grégory Chatel, Vincent Pilaud \& Viviane Pons
}

\begin{abstract}
We explore lattice structures on integer binary relations (i.e. binary relations on the set $\{1,2, \ldots, n\}$ for a fixed integer $n$ ) and on integer posets (i.e. partial orders on the set $\{1,2, \ldots, n\}$ for a fixed integer $n$ ). We first observe that the weak order on the symmetric group naturally extends to a lattice structure on all integer binary relations. We then show that the subposet of this weak order induced by integer posets defines as well a lattice. We finally study the subposets of this weak order induced by specific families of integer posets corresponding to the elements, the intervals, and the faces of the permutahedron, the associahedron, and some recent generalizations of those.
\end{abstract}

The weak order is the lattice on the symmetric group $\mathfrak{S}(n)$ defined as the inclusion order of inversions, where an inversion of $\sigma \in \mathfrak{S}(n)$ is a pair of values $a<b$ such that $\sigma^{-1}(a)>\sigma^{-1}(b)$. It is a fundamental tool for the study of the symmetric group, in connection to reduced expressions of permutations as products of simple transpositions. Its Hasse diagram can also be seen as a certain acyclic orientation of the skeleton of the permutahedron (the convex hull of all permutations of $\mathfrak{S}(n)$ seen as vectors in $\mathbb{R}^{n}$ ).

This paper extends the weak order to all integer binary relations, i.e. binary relations on the set $[n]:=\{1,2, \ldots, n\}$ for a fixed integer $n$. A permutation $\sigma \in \mathfrak{S}(n)$ is seen as an binary relation $\triangleleft$ on $[n]$ where $u \triangleleft v$ when $u$ appears before $v$ in $\sigma$. Inversions of $\sigma$ then translates to decreasing relations of $\triangleleft$, i.e. elements $a<b$ such that $b \triangleleft a$. This interpretation enables to naturally extend the weak order to all binary relations on $[n]$ as follows. For any two binary relations $\mathrm{R}, \mathrm{S}$ on $[n]$, we define

$$
\mathrm{R} \preccurlyeq \mathrm{S} \quad \Longleftrightarrow \quad \mathrm{R}^{\operatorname{lnc}} \supseteq \mathrm{S}^{\operatorname{lnc}} \text { and } \mathrm{R}^{\text {Dec }} \subseteq \mathrm{S}^{\text {Dec }},
$$

where $\mathrm{R}^{\operatorname{lnc}}:=\{(a, b) \in \mathrm{R} \mid a \leqslant b\}$ and $\mathrm{R}^{\text {Dec }}:=\{(b, a) \in \mathrm{R} \mid a \leqslant b\}$ respectively denote the increasing and decreasing subrelations of $\mathrm{R}$. We call this order the weak order on integer binary relations, see Figure 1. The central result of this paper is the following statement, see Figure 5.

TheOREM. For any $n \in \mathbb{N}$, the weak order restricted to the set of all posets on $[n]$ is a lattice.

Our motivation for this result is that many relevant combinatorial objects can be interpreted by specific integer posets, and the subposets of the weak order induced by these specific integer posets often correspond to classical lattice structures on these

Manuscript received 29th July 2017, revised 20th July 2018, accepted 23rd July 2018.

KEYWORDS. Integer binary relations, Weak order, Lattices.

ACKnowledgements. VPi was supported by the French ANR grant SC3A (15 CE40 0004 01). 
combinatorial objects. To illustrate this, we study specific integer posets corresponding to the elements, to the intervals, and to the faces in the classical weak order, the Tamari and Cambrian lattices $[10,16]$, the boolean lattice, and other related lattices defined in [14]. By this systematic approach, we rediscover and shed light on lattice structures studied by G. Chatel and V. Pons on Tamari interval posets [3], by G. Chatel and V. Pilaud on Cambrian and Schröder-Cambrian trees [2], by D. Krob, M. Latapy, J.-C. Novelli, H.-D. Phan and S. Schwer on pseudo-permutations [7], and by P. Palacios and M. Ronco [12] and J.-C. Novelli and J.-Y. Thibon [11] on plane trees.

The research code for experiments and computations along this work is available online [13].

\section{THE WEAK ORDER ON INTEGER POSETS}

\subsection{ThE WEAK ORDER ON INTEGER BINARY RELATIONS.}

1.1.1. Integer binary relations. Our main object of focus are binary relations on integers. An integer (binary) relation of size $n$ is a binary relation on $[n]:=\{1, \ldots, n\}$, that is, a subset $\mathrm{R}$ of $[n]^{2}$. As usual, we write equivalently $(u, v) \in \mathrm{R}$ or $u \mathrm{R} v$, and similarly, we write equivalently $(u, v) \notin \mathrm{R}$ or $u R v$. Recall that a relation $\mathrm{R} \in[n]^{2}$ is called:

- reflexive if $u \mathrm{R} u$ for all $u \in[n]$,

- transitive if $u \mathrm{R} v$ and $v \mathrm{R} w$ implies $u \mathrm{R} w$ for all $u, v, w \in[n]$,

- symmetric if $u \mathrm{R} v$ implies $v \mathrm{R} u$ for all $u, v \in[n]$,

- antisymmetric if $u \mathrm{R} v$ and $v \mathrm{R} u$ implies $u=v$ for all $u, v \in[n]$.

From now on, we only consider reflexive relations. We denote by $\mathcal{R}(n)(\operatorname{resp} . \mathcal{T}(n)$, resp. $\mathcal{S}(n)$, resp. $\mathcal{A}(n)$ ) the collection of all reflexive (resp. reflexive and transitive, resp. reflexive and symmetric, resp. reflexive and antisymmetric) integer relations of size $n$. We denote by $\mathcal{E}(n)$ the set of integer equivalences of size $n$, that is, reflexive transitive symmetric integer relations, and by $\mathcal{P}(n)$ the collection of integer posets of size $n$, that is, reflexive transitive antisymmetric integer relations. In all these notations, we forget the $n$ when we consider a relation without restriction on its size.

A subrelation of $\mathrm{R} \in \mathcal{R}(n)$ is a relation $\mathrm{S} \in \mathcal{R}(n)$ such that $\mathrm{S} \subseteq \mathrm{R}$ as subsets of $[n]^{2}$. We say that $\mathrm{S}$ coarsens $\mathrm{R}$ and $\mathrm{R}$ extends $\mathrm{S}$. The extension order defines a graded lattice structure on $\mathcal{R}(n)$ whose meet and join are respectively given by intersection and union. The complementation $\mathrm{R} \mapsto\{(u, v) \mid u=v$ or $u R v\}$ is an antiautomorphism of $(\mathcal{R}(n), \subseteq, \cap, \cup)$ and makes it an ortho-complemented lattice.

Note that $\mathcal{T}(n), \mathcal{S}(n)$ and $\mathcal{A}(n)$ are all stable by intersection, while only $\mathcal{S}(n)$ is stable by union. In other words, $(\mathcal{S}(n), \subseteq, \cap, \cup)$ is a sublattice of $(\mathcal{R}(n), \subseteq, \cap, \cup)$, while $(\mathcal{T}(n), \subseteq)$ and $(\mathcal{A}(n), \subseteq)$ are meet-semisublattice of $(\mathcal{R}(n), \subseteq, \cap)$ but not sublattices of $(\mathcal{R}(n), \subseteq, \cap, \cup)$. However, $(\mathcal{T}(n), \subseteq)$ is a lattice. To see it, consider the transitive closure of a relation $\mathrm{R} \in \mathcal{R}(n)$ defined by

$\mathrm{R}^{\mathrm{tc}}:=\left\{(u, w) \in[n]^{2} \mid \exists v_{1}, \ldots, v_{p} \in[n]\right.$ such that $\left.u=v_{1} \mathrm{R} v_{2} \mathrm{R} \ldots \mathrm{R} v_{p-1} \mathrm{R} v_{p}=w\right\}$.

The transitive closure $\mathrm{R}^{\mathrm{tc}}$ is the coarsest transitive relation containing $\mathrm{R}$. It follows that $(\mathcal{T}(n), \subseteq)$ is a lattice where the meet of $\mathrm{R}, \mathrm{S} \in \mathcal{R}(n)$ is given by $\mathrm{R} \cap \mathrm{S}$ and the join of $\mathrm{R}, \mathrm{S} \in \mathcal{R}(n)$ is given by $(\mathrm{R} \cup \mathrm{S})^{\mathrm{tc}}$. Since the transitive closure preserves symmetry, the subposet $(\mathcal{E}(n), \subseteq)$ of integer equivalences is a sublattice of $(\mathcal{T}(n), \subseteq)$.

1.1.2. Weak order. From now on, we consider both a relation $\mathrm{R}$ and the natural order $<$ on $[n]$ simultaneously. To limit confusions, we try to stick to the following convention throughout the paper. We denote integers by letters $a, b, c$ when we know 
that $a<b<c$ in the natural order. In contrast, we prefer to denote integers by letters $u, v, w$ when we do not know their relative order. This only helps avoid confusions and is always specified.

Let $\mathrm{I}_{n}:=\left\{(a, b) \in[n]^{2} \mid a \leqslant b\right\}$ and $\mathrm{D}_{n}:=\left\{(b, a) \in[n]^{2} \mid a \leqslant b\right\}$. Observe that $\mathrm{I}_{n} \cup \mathrm{D}_{n}=[n]^{2}$ while $\mathrm{I}_{n} \cap \mathrm{D}_{n}=\{(a, a) \mid a \in[n]\}$. We say that the relation $\mathrm{R} \in \mathcal{R}(n)$ is increasing (resp. decreasing) when $\mathrm{R} \subseteq \mathrm{I}_{n}$ (resp. $\mathrm{R} \subseteq \mathrm{D}_{n}$ ). We denote by $\mathcal{I}(n)$ (resp. $\mathcal{D}(n)$ ) the collection of all increasing (resp. decreasing) relations on $[n]$. The increasing and decreasing subrelations of an integer relation $\mathrm{R} \in \mathcal{R}(n)$ are the relations defined by

$$
\begin{array}{ll} 
& \mathrm{R}^{\operatorname{lnc}}:=\mathrm{R} \cap \mathrm{I}_{n}=\{(a, b) \in \mathrm{R} \mid a \leqslant b\} \in \mathcal{I}(n) \\
\text { and } & \mathrm{R}^{\mathrm{Dec}}:=\mathrm{R} \cap \mathrm{D}_{n}=\{(b, a) \in \mathrm{R} \mid a \leqslant b\} \in \mathcal{D}(n) .
\end{array}
$$

In our pictures, we always represent an integer relation $\mathrm{R} \in \mathcal{R}(n)$ as follows: we write the numbers $1, \ldots, n$ from left to right and we draw the increasing relations of $\mathrm{R}$ above in blue and the decreasing relations of $\mathrm{R}$ below in red. Although we only consider reflexive relations, we always omit the relations $(i, i)$ in the pictures (as well as in our explicit examples). See e.g. Figure 1.

Besides the extension lattice mentioned above in Section 1.1.1, there is another natural poset structure on $\mathcal{R}(n)$, whose name will be justified in Section 2.1.

DeFinition 1.1. The weak order on $\mathcal{R}(n)$ is the order defined by $\mathrm{R} \preccurlyeq \mathrm{S}$ if and only if $\mathrm{R}^{\operatorname{lnc}} \supseteq \mathrm{S}^{\operatorname{lnc}}$ and $\mathrm{R}^{\text {Dec }} \subseteq \mathrm{S}^{\text {Dec }}$

The weak order on $\mathcal{R}(3)$ is illustrated in Figure 1. Observe that the weak order is obtained by combining the extension lattice on increasing subrelations with the coarsening lattice on decreasing subrelations. In other words, $\mathcal{R}(n)$ is the square of an $\left(\begin{array}{l}n \\ 2\end{array}\right)$-dimensional boolean lattice. It explains the following statement.

Proposition 1.2. The weak order on $\mathcal{R}(n)$ is a graded lattice whose meet and join are given by

$$
\begin{aligned}
\mathrm{R} \wedge_{\mathcal{R}} \mathrm{S} & =\left(\mathrm{R}^{\mathrm{Inc}} \cup \mathrm{S}^{\mathrm{Inc}}\right) \cup\left(\mathrm{R}^{\mathrm{Dec}} \cap \mathrm{S}^{\mathrm{Dec}}\right) \\
\text { and } \quad \mathrm{R} \vee_{\mathcal{R}} \mathrm{S} & =\left(\mathrm{R}^{\mathrm{Inc}} \cap \mathrm{S}^{\mathrm{Inc}}\right) \cup\left(\mathrm{R}^{\mathrm{Dec}} \cup \mathrm{S}^{\mathrm{Dec}}\right) .
\end{aligned}
$$

Proof. The weak order is clearly a poset (the antisymmetry comes from the fact that $\left.\mathrm{R}=\mathrm{R}^{\operatorname{lnc}} \cup \mathrm{R}^{\mathrm{Dec}}\right)$. Its cover relations are all of the form $\mathrm{R} \preccurlyeq \mathrm{R} \backslash\{(a, b)\}$ for $a \mathrm{R}^{\text {Inc }} b$ or $\mathrm{R} \backslash\{(b, a)\} \preccurlyeq \mathrm{R}$ with $b \mathrm{R}^{\text {Dec }} a$. Therefore, the weak order is graded by $\mathrm{R} \mapsto\left|\mathrm{R}^{\text {Dec }}\right|-\left|\mathrm{R}^{\text {Inc }}\right|$. To check that it is a lattice, consider $\mathrm{R}, \mathrm{S} \in \mathcal{R}(n)$. Observe first that $\mathrm{R} \wedge_{\mathcal{R}} \mathrm{S}$ is indeed below both $\mathrm{R}$ and $\mathrm{S}$ in weak order. Moreover, if $\mathrm{T} \preccurlyeq \mathrm{R}$ and $\mathrm{T} \preccurlyeq \mathrm{S}$, then $\mathrm{T}^{\mathrm{Inc}} \supseteq \mathrm{R}^{\mathrm{Inc}} \cup \mathrm{S}^{\text {Inc }}$ and $\mathrm{T}^{\mathrm{Dec}} \subseteq \mathrm{R}^{\mathrm{Dec}} \cap \mathrm{S}^{\mathrm{Dec}}$, so that $\mathrm{T} \preccurlyeq \mathrm{R} \wedge_{\mathcal{R}} \mathrm{S}$. This proves that $\mathrm{R} \wedge_{\mathcal{R}} \mathrm{S}$ is indeed the meet of $\mathrm{R}$ and $\mathrm{S}$. The proof is similar for the join.

REMARK 1.3. Let $\mathrm{R}^{\text {rev }}:=\left\{(u, v) \in[n]^{2} \mid(v, u) \in \mathrm{R}\right\}$ denote the reverse of a relation $\mathrm{R} \in \mathcal{R}$. Note that $\left(\mathrm{R}^{\text {rev }}\right)^{\operatorname{lnc}}=\left(\mathrm{R}^{\mathrm{Dec}}\right)^{\text {rev }}$ and $\left(\mathrm{R}^{\text {rev }}\right)^{\text {Dec }}=\left(\mathrm{R}^{\text {Inc }}\right)^{\text {rev }}$. Therefore, the reverse map $\mathrm{R} \mapsto \mathrm{R}^{\text {rev }}$ defines an antiautomorphism of the weak or$\operatorname{der}\left(\mathcal{R}(n), \preccurlyeq, \wedge_{\mathcal{R}}, \vee_{\mathcal{R}}\right)$. Note that it preserves symmetry, antisymmetry and transitivity.

1.2. THE WEAK ORDER ON INTEGER POSETS. In this section, we show that the three subposets of the weak order on $\mathcal{R}(n)$ induced by antisymmetric relations, by transitive relations, and by posets are all lattices (although the last two are not sublattices of the weak order on $\mathcal{R}(n))$. 


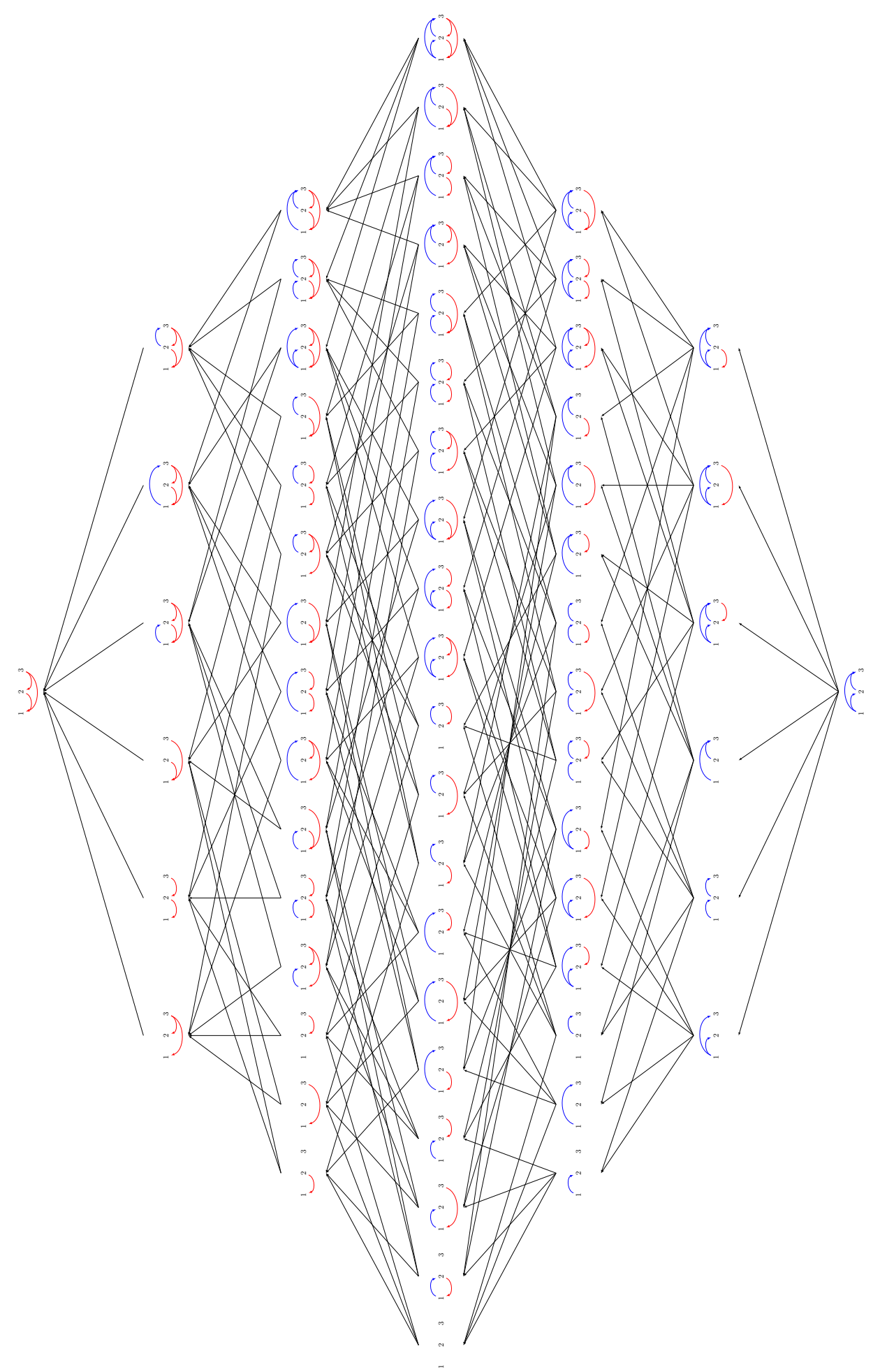

FiguRE 1. The weak order on (reflexive) integer binary relations of size 3. All reflexive relations $(i, i)$ for $i \in[n]$ are omitted. 
1.2.1. Antisymmetric relations. We first treat the case of antisymmetric relations. Figure 2 shows the meet and join of two antisymmetric relations, and illustrates the following statement.

Proposition 1.4. The meet $\wedge_{\mathcal{R}}$ and the join $\vee_{\mathcal{R}}$ both preserve antisymmetry. Thus, the set $\mathcal{A}(n)$ induces a sublattice of the weak order on $\mathcal{R}(n)$.

Proof. Let $\mathrm{R}, \mathrm{S} \in \mathcal{A}(n)$. Let $a<b \in[n]$ be such that $(b, a) \in \mathrm{R} \wedge_{\mathcal{R}} \mathrm{S}$. Since $(b, a)$ is decreasing and $\left(\mathrm{R} \wedge_{\mathcal{R}} \mathrm{S}\right)^{\text {Dec }}=\mathrm{R}^{\text {Dec }} \cap \mathrm{S}^{\text {Dec }}$, we have $b \mathrm{R}^{\text {Dec }} a$ and $b \mathrm{~S}^{\text {Dec }} a$. By antisymmetry of $\mathrm{R}$ and $\mathrm{S}$, we obtain that $a \mathbb{R}^{\operatorname{lnc}} b$ and $a \mathrm{~S}^{\operatorname{lnc}} b$. Therefore, $(a, b) \notin \mathrm{R}^{\operatorname{lnc}} \cup \mathrm{S}^{\operatorname{lnc}}=\left(\mathrm{R} \wedge_{\mathcal{R}} \mathrm{S}\right)^{\operatorname{lnc}}$. We conclude that $(b, a) \in \mathrm{R} \wedge_{\mathcal{R}} \mathrm{S}$ implies $(a, b) \notin \mathrm{R} \wedge_{\mathcal{R}} \mathrm{S}$ and thus that $\mathrm{R} \wedge_{\mathcal{R}} \mathrm{S}$ is antisymetric. The proof is identical for $\vee_{\mathcal{R}}$.

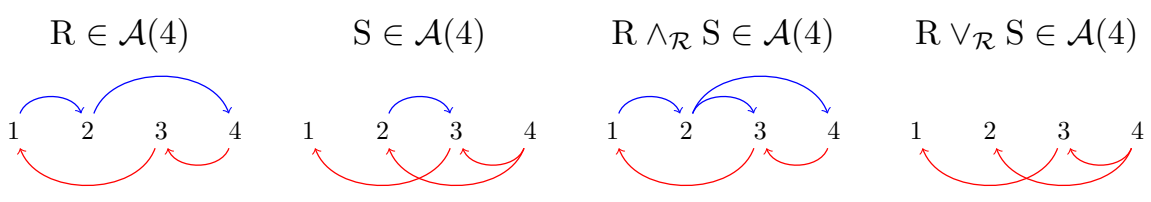

Figure 2. Two antisymmetric relations $\mathrm{R}, \mathrm{S} \in \mathcal{A}(4)$ (left) and heir meet $\mathrm{R} \wedge_{\mathcal{R}} \mathrm{S}$ and join $\mathrm{R} \vee_{\mathcal{R}} \mathrm{S}$ (right).

Our next two statements describe all cover relations in the weak order on $\mathcal{A}(n)$.

Proposition 1.5. All cover relations in $(\mathcal{A}(n), \preccurlyeq)$ are cover relations in $(\mathcal{R}(n), \preccurlyeq)$. In particular, the weak order on $\mathcal{A}(n)$ is still graded by $\mathrm{R} \mapsto\left|\mathrm{R}^{\mathrm{Dec}}\right|-\left|\mathrm{R}^{\text {Inc }}\right|$.

Proof. Consider a cover relation $\mathrm{R} \preccurlyeq \mathrm{S}$ in $(\mathcal{A}(n), \preccurlyeq)$. We have $\mathrm{R}^{\operatorname{lnc}} \supseteq \mathrm{S}^{\operatorname{lnc}}$ and $\mathrm{R}^{\text {Dec }} \subseteq \mathrm{S}^{\text {Dec }}$ where at least one of the inclusions is strict. Suppose first that $\mathrm{R}^{\operatorname{lnc}} \neq \mathrm{S}^{\operatorname{lnc}}$. Let $(a, b) \in \mathrm{R}^{\operatorname{lnc}} \backslash \mathrm{S}^{\operatorname{lnc}}$ and $\mathrm{T}:=\mathrm{R} \backslash\{(a, b)\}$. Note that $\mathrm{T}$ is still antisymmetric as it is obtained by removing an arc from an antisymmetric relation. Moreover, we have $\mathrm{R} \neq \mathrm{T}$ and $\mathrm{R} \preccurlyeq \mathrm{T} \preccurlyeq \mathrm{S}$. Since $\mathrm{S}$ covers $\mathrm{R}$, this implies that $\mathrm{S}=\mathrm{T}=\mathrm{R} \backslash\{(a, b)\}$. We prove similarly that if $\mathrm{R}^{\text {Dec }} \neq \mathrm{S}^{\text {Dec }}$, there exists $a<b$ such that $\mathrm{S}=\mathrm{R} \cup\{(b, a)\}$. In both cases, $\mathrm{R} \preccurlyeq \mathrm{S}$ is a cover relation in $(\mathcal{R}(n), \preccurlyeq)$.

COROLlARY 1.6. In the weak order on $\mathcal{A}(n)$, the antisymmetric relations that cover a given antisymmetric relation $\mathrm{R} \in \mathcal{A}(n)$ are precisely the relations

- $\mathrm{R} \backslash\{(a, b)\}$ for $a<b$ such that $a \mathrm{R} b$,

- $\mathrm{R} \cup\{(b, a)\}$ for $a<b$ such that $a \mathbb{R} b$ and $b \not R a$.

1.2.2. Transitive relations. We now consider transitive relations. Observe first that the subposet of the weak order on $\mathcal{R}(n)$ induced by the set $\mathcal{T}(n)$ is not a sublattice since $\wedge_{\mathcal{R}}$ and $\vee_{\mathcal{R}}$ do not preserve transitivity (see e.g. Figure 4 ). When R and $\mathrm{S}$ are transitive, we need to transform $\mathrm{R} \wedge_{\mathcal{R}} \mathrm{S}$ to make it a transitive relation $\mathrm{R} \wedge_{\mathcal{T}} \mathrm{S}$. We proceed in two steps described below.

Semitransitive relations. Before dealing with transitive relations, we introduce the intermediate notion of semitransitivity. We say that a relation $\mathrm{R} \in \mathcal{R}$ is semitransitive when both $\mathrm{R}^{\mathrm{Inc}}$ and $\mathrm{R}^{\mathrm{Dec}}$ are transitive. We denote by $\mathcal{S} \mathcal{T}(n)$ the collection of all semitransitive relations of size $n$. Figure 3 illustrates the following statement. 


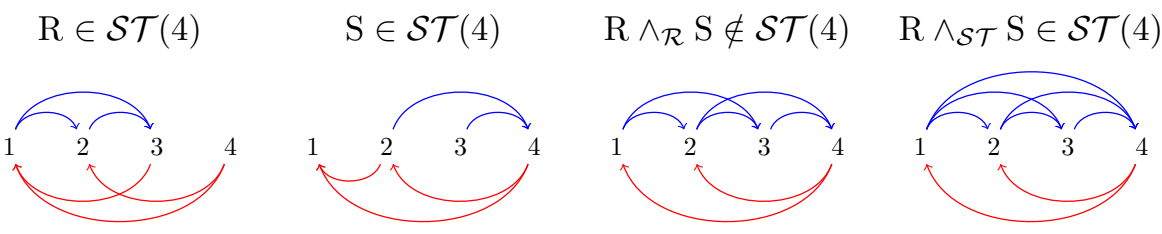

Figure 3. Two semi-transitive relations $\mathrm{R}, \mathrm{S} \in \mathcal{S} \mathcal{T}(4)$ (left) and their meets $\mathrm{R} \wedge_{\mathcal{R}} \mathrm{S}$ and $\mathrm{R} \wedge_{\mathcal{S} \mathcal{T}} \mathrm{S}$ (right).

Proposition 1.7. The weak order on $\mathcal{S T}(n)$ is a lattice whose meet and join are given by

$$
\begin{aligned}
\mathrm{R} \wedge_{\mathcal{S T}} \mathrm{S} & =\left(\mathrm{R}^{\mathrm{lnc}} \cup \mathrm{S}^{\mathrm{lnc}}\right)^{\mathrm{tc}} \cup\left(\mathrm{R}^{\mathrm{Dec}} \cap \mathrm{S}^{\mathrm{Dec}}\right) \\
\text { and } \quad \mathrm{R} \vee_{\mathcal{S T}} \mathrm{S} & =\left(\mathrm{R}^{\mathrm{lnc}} \cap \mathrm{S}^{\mathrm{lnc}}\right) \cup\left(\mathrm{R}^{\mathrm{Dec}} \cup \mathrm{S}^{\text {Dec }}\right)^{\mathrm{tc}} .
\end{aligned}
$$

Proof. Let $\mathrm{R}, \mathrm{S} \in \mathcal{S} \mathcal{T}(n)$. Observe first that $\mathrm{R} \wedge_{\mathcal{S} \mathcal{T}} \mathrm{S}$ is indeed semitransitive and below both $\mathrm{R}$ and $\mathrm{S}$. Moreover, if a semitransitive relation $\mathrm{T}$ is such that $\mathrm{T} \preccurlyeq \mathrm{R}$ and $\mathrm{T} \preccurlyeq \mathrm{S}$, then $\mathrm{T}^{\text {Inc }} \supseteq \mathrm{R}^{\text {Inc }} \cup \mathrm{S}^{\text {Inc }}$ and $\mathrm{T}^{\text {Dec }} \subseteq \mathrm{R}^{\text {Dec }} \cap \mathrm{S}^{\text {Dec }}$. By semitransitivity of $\mathrm{T}$, we get $\mathrm{T}^{\text {Inc }} \supseteq\left(\mathrm{R}^{\text {Inc }} \cup \mathrm{S}^{\text {Inc }}\right)^{\text {tc }}$, so that $\mathrm{T} \preccurlyeq \mathrm{R} \wedge_{\mathcal{S} \mathcal{T}} \mathrm{S}$. This proves that $\mathrm{R} \wedge_{\mathcal{S} \mathcal{T}} \mathrm{S}$ is indeed the meet of $\mathrm{R}$ and $\mathrm{S}$. The proof is similar for the join.

As in the previous section, we describe all cover relations in the weak order on $\mathcal{S T}(n)$.

PROPOSITION 1.8. All cover relations in $(\mathcal{S T}(n), \preccurlyeq)$ are cover relations in $(\mathcal{R}(n), \preccurlyeq)$. In particular, the weak order on $\mathcal{S T}(n)$ is still graded by $\mathrm{R} \mapsto\left|\mathrm{R}^{\text {Dec }}\right|-\left|\mathrm{R}^{\text {Inc }}\right|$.

Proof. Consider a cover relation $\mathrm{R} \preccurlyeq \mathrm{S}$ in $(\mathcal{S T}(n), \preccurlyeq)$. We have $\mathrm{R}^{\operatorname{lnc}} \supseteq \mathrm{S}^{\operatorname{lnc}}$ and $\mathrm{R}^{\text {Dec }} \subseteq \mathrm{S}^{\text {Dec }}$ where at least one of the inclusions is strict. Suppose first that $\mathrm{R}^{\operatorname{lnc}} \neq \mathrm{S}^{\operatorname{lnc}}$. Let $(a, b) \in \mathrm{R}^{\operatorname{lnc}} \backslash \mathrm{S}^{\operatorname{lnc}}$ be such that $b-a$ is minimal, and let $\mathrm{T}:=\mathrm{R} \backslash\{(a, b)\}$. Observe that there is no $a<i<b$ such that $a \mathrm{R} i \mathrm{R} b$. Otherwise, by minimality of $b-a$, we would have $a \mathrm{~S} i$ and $i \mathrm{~S} b$ while $a \not b$, contradicting the transitivity of $S^{\text {Inc }}$. It follows that $T^{\text {Inc }}$ is still transitive. Since $T^{\text {Dec }}=R^{\text {Dec }}$ is also transitive, we obtain that $\mathrm{T}$ is semitransitive. Moreover, we have $\mathrm{R} \neq \mathrm{T}$ and $\mathrm{R} \preccurlyeq \mathrm{T} \preccurlyeq \mathrm{S}$. Since $\mathrm{S}$ covers $\mathrm{R}$, this implies that $\mathrm{S}=\mathrm{T}=\mathrm{R} \backslash\{(a, b)\}$. We prove similarly that if $\mathrm{R}^{\mathrm{Dec}} \neq \mathrm{S}^{\text {Dec }}$, there exists $(b, a)$ such that $\mathrm{S}=\mathrm{R} \cup\{(b, a)\}$ : in this case, one needs to pick $(b, a) \in \mathrm{S}^{\text {Dec }} \backslash \mathrm{R}^{\text {Dec }}$ with $b-a$ maximal. In both cases, $\mathrm{R} \preccurlyeq \mathrm{S}$ is a cover relation in $(\mathcal{R}(n), \preccurlyeq)$.

COROLLARY 1.9. In the weak order on $\mathcal{S T}(n)$, the semitransitive relations that cover a given semitransitive relation $\mathrm{R} \in \mathcal{S} \mathcal{T}(n)$ are precisely the relations

- $\mathrm{R} \backslash\{(a, b)\}$ for $a<b$ such that $a \mathrm{R} b$ and there is no $a<i<b$ with $a \mathrm{R} i \mathrm{R} b$,

- $\mathrm{R} \cup\{(b, a)\}$ for $a<b$ such that $b \not R$ and there is no $i<a$ with $a \mathrm{R} i$ but $b \mathbb{R} i$ and similarly no $b<j$ with $j \mathrm{R} b$ but $j \not R$.

Transitive relations. Now we consider transitive relations. Observe that $\mathcal{T}(n) \subseteq \mathcal{S T}(n)$ but $\mathcal{S} \mathcal{T}(n) \nsubseteq \mathcal{T}(n)$. In particular, $\mathrm{R} \wedge_{\mathcal{S}} \mathcal{S}$ and $\mathrm{R} \vee_{\mathcal{S T}} \mathrm{S}$ may not be transitive even if $\mathrm{R}$ and $\mathrm{S}$ are (see Figure 4 ). To see that the subposet of the weak order induced by transitive relations is indeed a lattice, we therefore need operations which ensure transitivity and are compatible with the weak order. For $\mathrm{R} \in \mathcal{R}$, define the transitive decreasing deletion of $\mathrm{R}$ as

$$
\mathrm{R}^{\text {tdd }}:=\mathrm{R} \backslash\left\{(b, a) \in \mathrm{R}^{\text {Dec }} \mid \exists i \leqslant b \text { and } j \geqslant a \text { such that } i \mathrm{R} b \mathrm{R} a \mathrm{R} j \text { while } i \not R j\right\},
$$


and the transitive increasing deletion of $\mathrm{R}$ as

$$
\mathrm{R}^{\text {tid }}:=\mathrm{R} \backslash\left\{(a, b) \in \mathrm{R}^{\operatorname{lnc}} \mid \exists i \geqslant a \text { and } j \leqslant b \text { such that } i \mathrm{R} a \mathrm{R} b \mathrm{R} j \text { while } i \not R j\right\} .
$$

Note that in these definitions, $i$ and $j$ may coincide with $a$ and $b$ (since we assumed that all our relations are reflexive). Figure 4 illustrates the transitive decreasing deletion: the rightmost relation $\mathrm{R} \wedge_{\mathcal{T}} \mathrm{S}$ is indeed obtained as $\left(\mathrm{R} \wedge_{\mathcal{S T}} \mathrm{S}\right)^{\text {tdd }}$. Observe that two decreasing relations have been deleted: $(3,1)$ (take $i=2$ and $j=1$, or $i=3$ and $j=2$ ) and $(4,1)$ (take $i=4$ and $j=2$ ).

REMARK 1.10. The idea of the transitive decreasing deletion is to delete all decreasing relations which prevent the binary relation to be transitive. It may thus seem more natural to assume in the definition of $\mathrm{R}^{\text {tdd }}$ that either $i=b$ or $j=a$. However, this would not suffice to rule out all non-transitive relations, consider for example the relation $[4]^{2} \backslash\{(2,3),(3,2)\}$. We would therefore need to iterate the deletion process, which would require to prove a converging property. Our definition of $\mathrm{R}^{\text {tdd }}$ simplifies the presentation as it requires only one deletion step.

Lemma 1.11. For any relation $\mathrm{R} \in \mathcal{R}$, we have $\mathrm{R}^{\mathrm{tdd}} \preccurlyeq \mathrm{R} \preccurlyeq \mathrm{R}^{\mathrm{tid}}$.

Proof. $\mathrm{R}^{\text {tdd }}$ is obtained from $\mathrm{R}$ by deleting decreasing relations. Therefore we have $\left(\mathrm{R}^{\text {tdd }}\right)^{\text {Inc }}=\mathrm{R}^{\text {Inc }}$ and $\left(\mathrm{R}^{\text {tdd }}\right)^{\text {Dec }} \subseteq \mathrm{R}^{\text {Dec }}$ and thus $\mathrm{R}^{\text {tdd }} \preccurlyeq \mathrm{R}$ by definition of the weak order. The argument is similar for $\mathrm{R}^{\text {tid }}$.

LEMMA 1.12. If $\mathrm{R} \in \mathcal{R}$ is semitransitive, then $\mathrm{R}^{\text {tdd }}$ and $\mathrm{R}^{\text {tid }}$ are transitive.

Proof. We prove the result for $\mathrm{R}^{\text {tdd }}$, the proof being symmetric for $\mathrm{R}^{\text {tid }}$. Set

$$
\mathrm{U}:=\left\{(b, a) \in \mathrm{R}^{\text {Dec }} \mid \exists i \leqslant b \text { and } j \geqslant a \text { such that } i \mathrm{R} b \mathrm{R} a \mathrm{R} j \text { while } i \not R j\right\},
$$

so that $\mathrm{R}^{\text {tdd }}=\mathrm{R} \backslash \mathrm{U}$ with $\left(\mathrm{R}^{\text {tdd }}\right)^{\text {Inc }}=\mathrm{R}^{\text {Inc }}$ and $\left(\mathrm{R}^{\text {tdd }}\right)^{\text {Dec }}=\mathrm{R}^{\text {Dec }} \backslash \mathrm{U}$. Let $u, v, w \in[n]$ be such that $u \mathrm{R}^{\text {tdd }} v$ and $v \mathrm{R}^{\text {tdd }} w$. We want to prove that $u \mathrm{R}^{\text {tdd }} w$. We distinguish six cases according to the relative order of $u, v, w$ :

(1) If $u<v<w$, then $u \mathrm{R}^{\text {Inc }} v$ and $v \mathrm{R}^{\text {Inc }} w$. Thus $u \mathrm{R}^{\text {Inc }} w$ by transitivity of $\mathrm{R}^{\text {Inc }}$, and thus $u \mathrm{R}^{\text {tdd }} w$.

(2) If $u<w<v$, then $u \mathrm{R}^{\text {Inc }} v$ and $v \mathrm{R}^{\text {Dec }} w$. Since $v \bigcup t$, we have $u \mathrm{R}^{\text {Inc }} w$ and thus $u \mathrm{R}^{\text {tdd }} w$.

(3) If $v<u<w$, then $u \mathrm{R}^{\text {Dec }} v$ and $v \mathrm{R}^{\operatorname{lnc}} w$. Since $u \emptyset v$, we have $u \mathrm{R}^{\operatorname{lnc}} w$ and thus $u \mathrm{R}^{\text {tdd }} w$.

(4) If $v<w<u$, then $u \mathrm{R}^{\text {Dec }} v$ and $v \mathrm{R}^{\operatorname{lnc}} w$. Since $u \bigvee v$, we have $u \mathrm{R}^{\text {Dec }} w$. Assume by contradiction that $u \mathrm{U} w$. Hence there is $i \leqslant u$ and $j \geqslant w$ such that $i \mathrm{R} u \mathrm{R} w \mathrm{R} j$ but $i \not R j$. Since $v \mathrm{R}^{\operatorname{lnc}} w$ and $w \mathrm{R}^{\operatorname{lnc}} j$, the transitivity of $\mathrm{R}^{\operatorname{lnc}}$ ensures that $v \mathrm{R} j$. We obtain that $u \mathrm{U} v$, a contradiction. Therefore, $u \bigvee w$ and $u \mathrm{R}^{\mathrm{tdd}} w$.

(5) If $w<u<v$, then $u \mathrm{R}^{\operatorname{lnc}} v$ and $v \mathrm{R}^{\text {Dec }} w$. Since $v \varnothing w$, we have $u \mathrm{R}^{\text {Dec }} w$. Assume by contradiction that $u \mathrm{U} w$. Hence there is $i \leqslant u$ and $j \geqslant w$ such that $i \mathrm{R} u \mathrm{R} w \mathrm{R} j$ but $i \not R j$. Since $i \mathrm{R}^{\operatorname{lnc}} u$ and $u \mathrm{R}^{\operatorname{lnc}} v$, the transitivity of $\mathrm{R}^{\operatorname{lnc}}$ ensures that $i \mathrm{R} v$. We obtain that $v \mathrm{U} w$, a contradiction. Therefore, $u \bigvee w$ and $u \mathrm{R}^{\text {tdd }} w$.

(6) If $w<v<u$, then $u \mathrm{R}^{\text {Dec }} v$ and $v \mathrm{R}^{\text {Dec }} w$, so that $u \mathrm{R}^{\text {Dec }} w$ by transitivity of $\mathrm{R}^{\mathrm{Dec}}$. Assume by contradiction that $u \mathrm{U} w$. Hence there is $i \leqslant u$ and $j \geqslant w$ such that $i \mathrm{R} u \mathrm{R} w \mathrm{R} j$ but $i \not R j$. Since $u \bigvee v$ and $v \not w$, we obtain that $i \mathrm{R} v$ and $v \mathrm{R} j$. If $i \leqslant v$, then we have $i \leqslant v$ and $j \geqslant w$ with $i \mathrm{R} v \mathrm{R} w \mathrm{R} j$ and $i \not R j$ contradicting the fact that $v \not w$. Similarly, if $j \geqslant v$, we have $i \leqslant u$ and $j \geqslant v$ with $i \mathrm{R} u \mathrm{R} v \mathrm{R} j$ and $i \not R j$ contradicting the fact that $u \bigcup v$. Finally, if $j<v<i$, we have $i \mathrm{R}^{\text {Dec }} v \mathrm{R}^{\text {Dec }} j$ and $i \mathbb{R}^{\text {Dec }} j$ contradicting the transitivity of $\mathrm{R}^{\text {Dec }}$. 

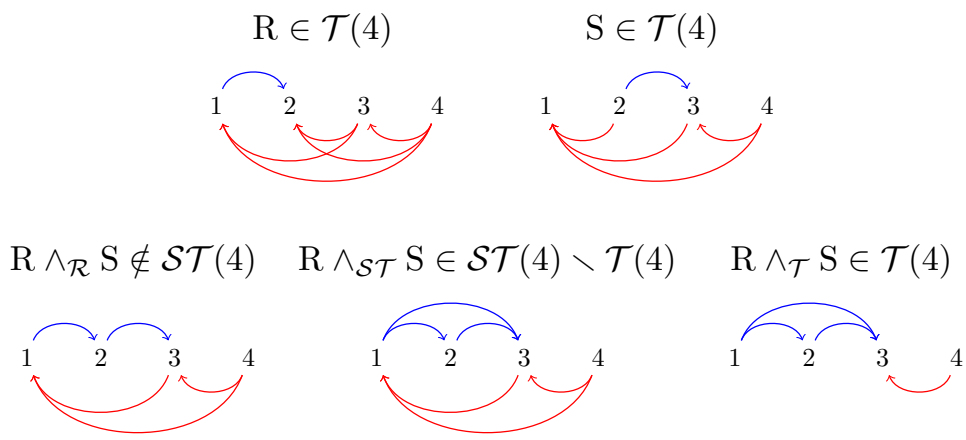

FiguRE 4. Two transitive relations $\mathrm{R}, \mathrm{S} \in \mathcal{T}(4)$ (top) and their meets $\mathrm{R} \wedge_{\mathcal{R}} \mathrm{S}, \mathrm{R} \wedge_{\mathcal{S} \mathcal{T}} \mathrm{S}$ and $\mathrm{R} \wedge_{\mathcal{T}} \mathrm{S}$ (bottom).

REMARK 1.13. We observed earlier that the transitive closure $\mathrm{R}^{\mathrm{tc}}$ is the coarsest transitive relation containing $R$. For $R \in \mathcal{S T}$, Lemmas 1.11 and 1.12 show that $R^{\text {tdd }}$ is a transitive relation below $\mathrm{R}$ in weak order. However, there might be other transitive relations $\mathrm{S}$ with $\mathrm{S} \preccurlyeq \mathrm{R}$ and which are not comparable to $\mathrm{R}^{\text {tdd }}$ in weak order. For example, consider $\mathrm{R}:=\{(1,3),(3,2)\}$ and $\mathrm{S}:=\{(1,2),(1,3),(3,2)\}$. Then $\mathrm{S}$ is transitive and $\mathrm{S} \preccurlyeq \mathrm{R}$ while $\mathrm{S}$ is incomparable to $\mathrm{R}^{\text {tdd }}=\{(1,3)\}$ in weak order.

We use the maps $\mathrm{R} \mapsto \mathrm{R}^{\text {tdd }}$ and $\mathrm{R} \mapsto \mathrm{R}^{\text {tid }}$ to obtain the main result of this section. Figure 4 illustrates all steps of a meet computation in $\mathcal{T}(4)$.

Proposition 1.14. The weak order on $\mathcal{T}(n)$ is a lattice whose meet and join are given by

$$
\begin{aligned}
\mathrm{R} \wedge_{\mathcal{T}} \mathrm{S} & =\left(\left(\mathrm{R}^{\operatorname{lnc}} \cup \mathrm{S}^{\mathrm{lnc}}\right)^{\mathrm{tc}} \cup\left(\mathrm{R}^{\mathrm{Dec}} \cap \mathrm{S}^{\mathrm{Dec}}\right)\right)^{\mathrm{tdd}} \\
\text { and } \quad \mathrm{R} \vee_{\mathcal{T}} \mathrm{S} & =\left(\left(\mathrm{R}^{\operatorname{lnc}} \cap \mathrm{S}^{\operatorname{lnc}}\right) \cup\left(\mathrm{R}^{\mathrm{Dec}} \cup \mathrm{S}^{\mathrm{Dec}}\right)^{\mathrm{tc}}\right)^{\mathrm{tid}} .
\end{aligned}
$$

Before proving the proposition, we state the following technical lemma which we will used repeatedly in our proofs.

LEMMA 1.15. Let $\mathrm{R}$ and $\mathrm{S}$ be two transitive relations, let $\mathrm{M}=\mathrm{R} \wedge_{\mathcal{S T}} \mathrm{S}$, and let $1 \leqslant a<b \leqslant n$ such that $b \mathrm{M} a$ and $b \mathrm{XI}^{\mathrm{tdd}} a$. By definition of $\mathrm{M}^{\mathrm{tdd}}$, there exist $i \leqslant b$ and $j \geqslant a$ such that $i \mathrm{M} b \mathrm{M} a \mathrm{M} j$ while $i$ XI $j$. Then we have

- either $i \neq b$ or $j \neq a$,

- if $i \neq b$, there is $a<k<b$ such that $i \mathrm{M} k \mathrm{M} b$ with $(k, b) \in \mathrm{R} \cup \mathrm{S}$ and $k \mathrm{XI}^{\mathrm{tdd}} a$,

- if $j \neq a$, there is $a<k<b$ such that $a \mathrm{M} k \mathrm{M} j$ with $(a, k) \in \mathrm{R} \cup \mathrm{S}$ and $b \not \mathrm{I}^{\mathrm{tdd}} k$, Besides if $\mathrm{R}$ and $\mathrm{S}$ are also antisymmetric, then in both cases, $b \mathrm{XI}^{\mathrm{tdd}} k \mathrm{XI}^{\mathrm{tdd}} a$.

Proof. Since $b \mathrm{M} a$ and $i$ XI $j$, we cannot have both $i=b$ and $j=a$. By symmetry, we can assume that $i \neq b$. Since $(i, b) \in \mathrm{M}^{\mathrm{Inc}}=\left(\mathrm{R}^{\operatorname{lnc}} \cup \mathrm{S}^{\operatorname{lnc}}\right)^{\mathrm{tc}}$, there exists $i \leqslant k<b$ such that $(i, k) \in \mathrm{M}^{\mathrm{Inc}}$ and $(k, b) \in \mathrm{R}^{\operatorname{lnc}} \cup \mathrm{S}^{\operatorname{lnc}}$. Assume without loss of generality that $k \mathrm{R}^{\text {Inc }} b$. We obtain that $k \mathrm{R} b \mathrm{R} a$ and thus that $k \mathrm{R} a$ by transitivity of $\mathrm{R}$. We want to prove that $k>a$.

Assume that $k \leqslant a$. Then $(k, a) \in \mathrm{R}^{\mathrm{Inc}} \subseteq \mathrm{M}^{\mathrm{Inc}}$ and thus $i \mathrm{M}^{\text {Inc }} k \mathrm{M}^{\text {Inc }} a \mathrm{M}^{\text {Inc }} j$ while $i \mathrm{XI}^{\mathrm{Inc}} j$ contradicting the transitivity of $\mathrm{M}^{\mathrm{Inc}}$. We then have $a<k<b$. There is left to prove that $k \mathrm{XI}^{\text {tdd }} a$. Suppose that we have $k \mathrm{M}^{\mathrm{tdd}} a$, then we have $i \mathrm{M} k \mathrm{M} a \mathrm{M} j$ which implies $i \mathrm{M} j$ because $(k, a)$ is not deleted by the transitive decreasing deletion. This contradicts our initial statement $i \not X I$. 
Besides, if $\mathrm{R}$ is antisymmetric, then $k \mathrm{R} b$ implies $b \not R k$ which gives $b \not \mathrm{XI}^{\mathrm{tdd}} k$.

Proof of Proposition 1.14. The weak order on $\mathcal{T}(n)$ is a subposet of the weak order on $\mathcal{R}(n)$. It is clearly bounded: the weak order minimal transitive relation is $\mathrm{I}_{n}=\left\{(a, b) \in[n]^{2} \mid a \leqslant b\right\}$ while the weak order maximal transitive relation is $\mathrm{D}_{n}=\left\{(b, a) \in[n]^{2} \mid a \leqslant b\right\}$. Therefore, we only have to show that any two transitive relations admit a meet and a join. We prove the result for the meet, the proof for the join being symmetric.

Let $\mathrm{R}, \mathrm{S} \in \mathcal{T}(n)$ and $\mathrm{M}:=\mathrm{R} \wedge_{\mathcal{S} \mathcal{T}} \mathrm{S}=\left(\mathrm{R}^{\text {Inc }} \cup \mathrm{S}^{\text {Inc }}\right)^{\mathrm{tc}} \cup\left(\mathrm{R}^{\text {Dec }} \cap \mathrm{S}^{\text {Dec }}\right)$, so that $\mathrm{R} \wedge_{\mathcal{T}} \mathrm{S}=\mathrm{M}^{\text {tdd }}$. First we have $\mathrm{M} \preccurlyeq \mathrm{R}$ so that $\mathrm{R} \wedge_{\mathcal{T}} \mathrm{S}=\mathrm{M}^{\text {tdd }} \preccurlyeq \mathrm{M} \preccurlyeq \mathrm{R}$ by Lemma 1.11. Similarly, $\mathrm{R} \wedge_{\mathcal{T}} \mathrm{S} \preccurlyeq \mathrm{S}$. Moreover, $\mathrm{R} \wedge_{\mathcal{T}} \mathrm{S}$ is transitive by Lemma 1.12. It thus remains to show that $\mathrm{R} \wedge_{\mathcal{T}} \mathrm{S}$ is larger than any other transitive relation smaller than both $\mathrm{R}$ and S.

Consider thus another transitive relation $\mathrm{T} \in \mathcal{T}(n)$ such that $\mathrm{T} \preccurlyeq \mathrm{R}$ and $\mathrm{T} \preccurlyeq \mathrm{S}$. We need to show that $\mathrm{T} \preccurlyeq \mathrm{R} \wedge_{\mathcal{T}} \mathrm{S}=\mathrm{M}^{\text {tdd }}$. Observe that $\mathrm{T} \preccurlyeq \mathrm{M}$ since $\mathrm{T}$ is semitransitive and $\mathrm{M}=\mathrm{R} \wedge_{\mathcal{S T}} \mathrm{S}$ is larger than any semitransitive relation smaller than both $\mathrm{R}$ and S. It implies in particular that $\mathrm{T}^{\operatorname{lnc}} \supseteq \mathrm{M}^{\mathrm{Inc}}=\left(\mathrm{M}^{\mathrm{tdd}}\right)^{\operatorname{lnc}}$ and that $\mathrm{T}^{\text {Dec }} \subseteq \mathrm{M}^{\text {Dec }}$.

Assume by contradiction that $\mathrm{T} \npreceq \mathrm{M}^{\text {tdd }}$. Since $\mathrm{T}^{\mathrm{Inc}} \supseteq\left(\mathrm{M}^{\mathrm{tdd}}\right)^{\mathrm{Inc}}$, this means that there exist $(b, a) \in \mathrm{T}^{\text {Dec }} \backslash \mathrm{M}^{\text {tdd }}$. We choose $(b, a) \in \mathrm{T}^{\text {Dec }} \backslash \mathrm{M}^{\text {tdd }}$ such that $b-a$ is minimal. Since $\mathrm{T}^{\text {Dec }} \subseteq \mathrm{M}^{\text {Dec }}$, we have $(b, a) \in \mathrm{M}^{\text {Dec }} \backslash \mathrm{M}^{\text {tdd }}$. By definition of $\mathrm{M}^{\text {tdd }}$, there exists $i \leqslant b$ and $j \geqslant a$ such that $i \mathrm{M} b \mathrm{M} a \mathrm{M} j$ while $i$ XI $j$. We use Lemma 1.15 and assume without loss of generality that there is $a<k<b$ with $(k, b) \in \mathrm{R} \cup \mathrm{S}$ and $k \times \mathrm{XI}^{\mathrm{tdd}} a$. Since $(k, b) \in\left(\mathrm{R}^{\operatorname{lnc}} \cup \mathrm{S}^{\mathrm{Inc}}\right) \subseteq \mathrm{T}^{\mathrm{Inc}}$ and $b \mathrm{~T} a$ we have $k \mathrm{~T} a$ by transitivity of $\mathrm{T}$. Since $k>a$, we get $(k, a) \in \mathrm{T}^{\text {Dec }} \subseteq \mathrm{M}^{\text {Dec }}$. But by Lemma $1.15,(k, a) \notin \mathrm{M}^{\text {tdd }}$ : it has been deleted by the transitive decreasing deletion and thus contradicts the minimality of $b-a$.

Remark 1.16. In contrast to Propositions 1.5 and 1.8 and Corollaries 1.6 and 1.9, the cover relations in the weak order on $\mathcal{T}(n)$ are more complicated to describe. In fact, the weak order on $\mathcal{T}(n)$ is not graded as soon as $n \geqslant 3$. Indeed, consider the maximal chains from $\mathrm{I}_{3}$ to $\mathrm{D}_{3}$ in $\mathcal{T}(3)$. Those chains passing through the trivial reflexive relation $\{(i, i) \mid i \in[n]\}$ all have length 6 , while those passing through the full relation $[3]^{2}$ all have length 4 .

1.2.3. Integer posets. We finally arrive to the subposet of the weak order induced by integer posets. The weak order on $\mathcal{P}(3)$ is illustrated in Figure 5. We now have all tools to show Theorem announced in the introduction.

Proposition 1.17. The transitive meet $\wedge_{\mathcal{T}}$ and the transitive join $\vee_{\mathcal{T}}$ both preserve antisymmetry. In other words, $\mathcal{P}(n)$ induces a sublattice of the weak order on $\mathcal{T}(n)$.

Proof. Let $\mathrm{R}, \mathrm{S} \in \mathcal{P}(n)$. Let $\mathrm{M}:=\mathrm{R} \wedge_{\mathcal{S T}} \mathrm{S}=\left(\mathrm{R}^{\text {Inc }} \cup \mathrm{S}^{\mathrm{Inc}}\right)^{\mathrm{tc}} \cup\left(\mathrm{R}^{\mathrm{Dec}} \cap \mathrm{S}^{\mathrm{Dec}}\right)$, so that $\mathrm{R} \wedge_{\mathcal{T}} \mathrm{S}=\mathrm{M}^{\text {tdd }}$. Assume by contradiction that $\mathrm{M}^{\text {tdd }}$ is not antisymmetric. Let $a<c \in[n]$ be such that $\{(a, c),(c, a)\} \subseteq \mathrm{M}^{\text {tdd }}$ with $c-a$ minimal. Since $(c, a) \in\left(\mathrm{M}^{\text {tdd }}\right)^{\text {Dec }} \subseteq \mathrm{M}^{\text {Dec }}=\mathrm{R}^{\text {Dec }} \cap \mathrm{S}^{\text {Dec }}$, we have $(a, c) \notin \mathrm{R}^{\text {Inc }} \cup \mathrm{S}^{\text {Inc }}$ by antisymmetry of $\mathrm{R}$ and S. Since $(a, c) \in\left(\mathrm{R}^{\text {Inc }} \cup \mathrm{S}^{\text {Inc }}\right)^{\text {tc }} \backslash\left(\mathrm{R}^{\operatorname{lnc}} \cup \mathrm{S}^{\text {Inc }}\right)$, there exists $a<b<c$ such that $\{(a, b),(b, c)\} \subseteq\left(\mathrm{R}^{\text {Inc }} \cup \mathrm{S}^{\text {lnc }}\right)^{\text {tc }}$. Since $c \mathrm{M}^{\text {tdd }} a \mathrm{M}^{\text {tdd }} b$, we obtain by transitivity of $\mathrm{M}^{\text {tdd }}$ that $\{(b, c),(c, b)\} \subseteq \mathrm{M}^{\text {tdd }}$, contradicting the minimality of $c-a$.

REMARK 1.18. In contrast, there is no guarantee that the semitransitive meet of two transitive antisymmetric relations is antisymmetric. For example in Figure 4, R and $\mathrm{S}$ are antisymmetric but $\mathrm{M}=\mathrm{R} \wedge_{\mathcal{S} \mathcal{T}} \mathrm{S}$ is not as it contains both $(1,3)$ and $(3,1)$. However, the relation $(3,1)$ is removed by the transitive decreasing delation and the result $\mathrm{M}^{\text {tdd }}=\mathrm{R} \wedge_{\mathcal{T}} \mathrm{S}$ is antisymmetric. 


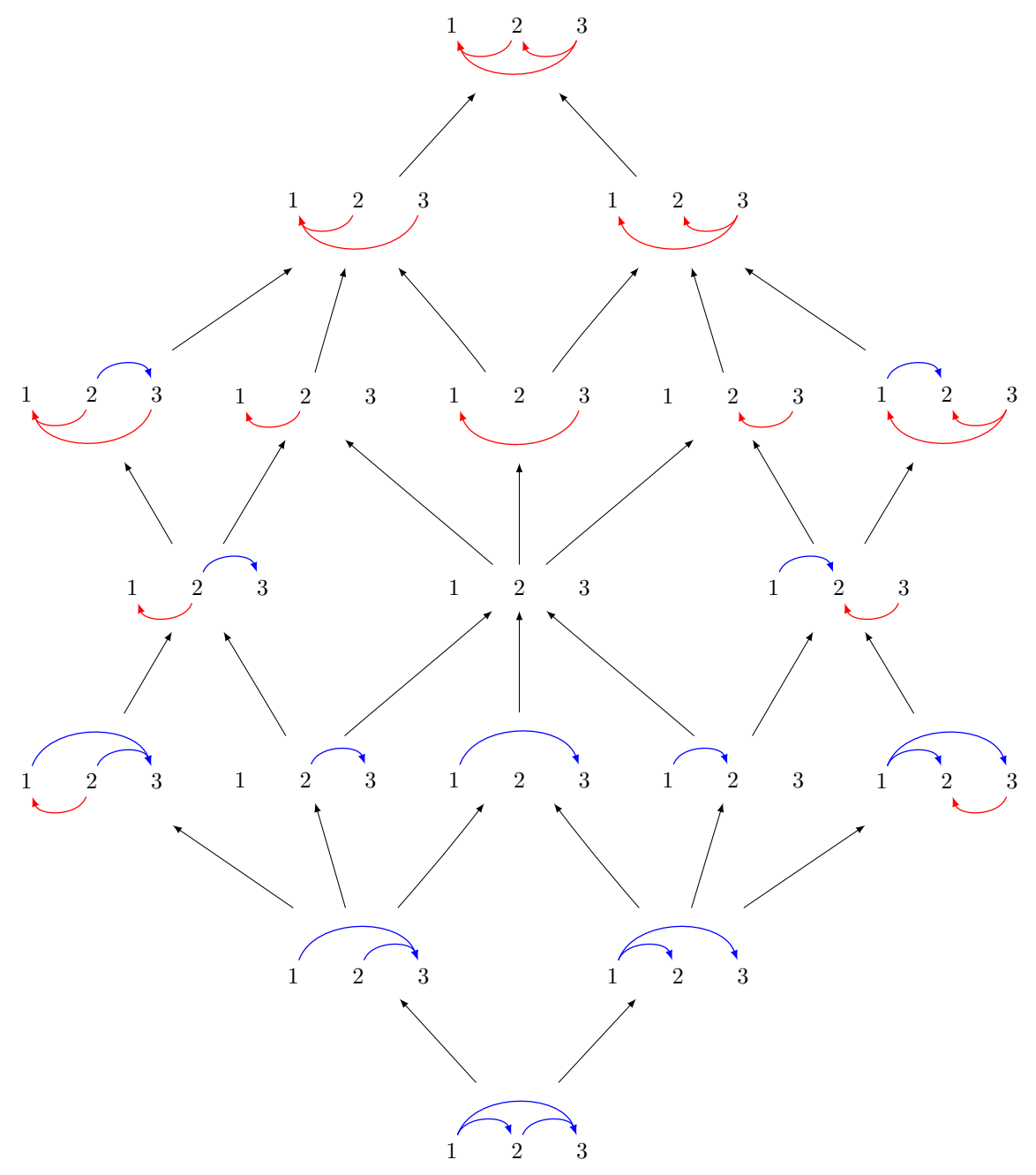

FigurE 5. The weak order on integer posets of size 3 .

As in Propositions 1.5 and 1.8 and Corollaries 1.6 and 1.9, the next two statements describe all cover relations in the weak order on $\mathcal{P}(n)$.

Proposition 1.19. All cover relations in $(\mathcal{P}(n), \preccurlyeq)$ are cover relations in $(\mathcal{R}(n), \preccurlyeq)$. In particular, the weak order on $\mathcal{P}(n)$ is still graded by $\mathrm{R} \mapsto\left|\mathrm{R}^{\mathrm{Dec}}\right|-\left|\mathrm{R}^{\text {Inc }}\right|$.

Proof. Consider a cover relation $\mathrm{R} \preccurlyeq \mathrm{S}$ in $(\mathcal{P}(n), \preccurlyeq)$. We have $\mathrm{R}^{\operatorname{lnc}} \supseteq \mathrm{S}^{\operatorname{lnc}}$ and $\mathrm{R}^{\text {Dec }} \subseteq \mathrm{S}^{\text {Dec }}$ where at least one of the inclusions is strict. Suppose first that $\mathrm{R}^{\operatorname{lnc}} \neq \mathrm{S}^{\operatorname{lnc}}$. Consider the set $\mathrm{X}:=\left\{(a, b) \in \mathrm{R}^{\operatorname{lnc}} \backslash \mathrm{S}^{\operatorname{lnc}} \mid \exists \exists a<i<b\right.$ with $\left.a \mathrm{R} i \mathrm{R} b\right\}$. This set $\mathrm{X}$ is nonempty as it contains any $(a, b)$ in $\mathrm{R}^{\operatorname{lnc}} \backslash \mathrm{S}^{\operatorname{lnc}}$ with $b-a$ minimal. Consider now $(a, b) \in \mathrm{X}$ with $b-a$ maximal and let $\mathrm{T}:=\mathrm{R} \backslash\{(a, b)\}$. We claim that $\mathrm{T}$ is still a poset. It is clearly still reflexive and antisymmetric. For transitivity, assume by means of contradiction that there is $j \in[n] \backslash\{a, b\}$ such that $a \mathrm{R} j \mathrm{R} b$. Since $(a, b) \in \mathrm{X}$, we know that $j<a$ or $b<j$. As these two options are symmetric, assume for instance that $j<a$ and choose $j$ so that $a-j$ is minimal. We claim that there is no $j<i<b$ such that $j \mathrm{R} i \mathrm{R} b$. Otherwise, since $a \mathrm{R} j \mathrm{R} i$ and $\mathrm{R}$ is transitive, we have $a \mathrm{R} i \mathrm{R} b$. 
Now, if $i<a$, we have $a \mathrm{R} i \mathrm{R} b$ and $j<i<a$ contradicting the minimality of $a-j$ in our choice of $j$. If $i>a$, we have $a \mathrm{R} i \mathrm{R} b$ and $a<i<b$ contradicting the fact that $(a, b) \in \mathrm{X}$. Finally, if $i=a$, we have $a \mathrm{R} j \mathrm{R} a$ contradicting the antisymmetry of $\mathrm{R}$. This proves that there is no $j<i<b$ such that $j \mathrm{R} i \mathrm{R} b$. By maximality of $b-a$ in our choice of $(a, b)$ this implies that $j \mathrm{~S} b$. Since $(a, j) \in \mathrm{R}^{\text {Dec }} \subseteq \mathrm{S}^{\text {Dec }}$, we therefore obtain that $a \mathrm{~S} j \mathrm{~S} b$ while $a \not b$, contradicting the transitivity of $\mathrm{S}$. This proves that $\mathrm{T}$ is transitive and it is thus a poset. Moreover, we have $\mathrm{R} \neq \mathrm{T}$ and $\mathrm{R} \preccurlyeq \mathrm{T} \preccurlyeq \mathrm{S}$. Since $\mathrm{S}$ covers $\mathrm{R}$, this implies that $\mathrm{S}=\mathrm{T}=\mathrm{R} \backslash\{(a, b)\}$. We prove similarly that if $\mathrm{R}^{\text {Dec }} \neq \mathrm{S}^{\text {Dec }}$, there exists $(b, a)$ such that $\mathrm{S}=\mathrm{R} \cup\{(b, a)\}$. In both cases, $\mathrm{R} \preccurlyeq \mathrm{S}$ is a cover relation in $(\mathcal{R}(n), \preccurlyeq)$.

COROLlaRY 1.20. In the weak order on $\mathcal{P}(n)$, the posets that cover a given integer poset $\mathrm{R} \in \mathcal{P}(n)$ are precisely the posets

- the relations $\mathrm{R} \backslash\{(a, b)\}$ for $a<b$ such that $a \mathrm{R} b$ and there is no $i \in[n]$ with $a \mathrm{R} i \mathrm{R} b$,

- the relations $\mathrm{R} \cup\{(b, a)\}$ for $a<b$ such that $a \not R b$ and $b \not R$ and there is no $i \neq a$ with $a \mathrm{R} i$ but $b \mathbb{R} i$ and similarly no $j \neq b$ with $j \mathrm{R} b$ but $j R$ a.

\section{WEAK ORDER INDUCED BY SOME RELEVANT FAMILIES OF POSETS}

In the rest of the paper, we present our motivation to study Theorem . We observe that many relevant combinatorial objects (for example permutations, binary trees, binary sequences, ...) can be interpreted by specific integer posets ${ }^{(1)}$. Moreover, the subposets of the weak order induced by these specific integer posets often correspond to classical lattice structures on these combinatorial objects (for example the classical weak order, the Tamari lattice, the boolean lattice,...). Table 1 summarizes the different combinatorial objects involved and a roadmap to their properties.

Rather than our previous notations R, S, M used for integer binary relations, we will denote integer posets by $\triangleleft, \triangleleft, \dashv$ so that $a \triangleleft b$ (resp. $a \triangleleft b$ and $a \dashv v$ ) means that $a$ is in relation with $b$ for the $\triangleleft$ relation. These notations emphasize the notion of order and allow us to write $a \triangleright b$ for $b \triangleleft a$, in particular when $a<b$. To make our presentation easier to read, we have decomposed some of our proofs into technical but straightforward claims that are proved separately in Appendix A.

2.1. From the Permutahedron. We start with relevant families of posets corresponding to the elements, the intervals, and the faces of the permutahedron. Further similar families of posets will appear in Sections 2.2 and 2.3.

Let $\mathfrak{S}(n)$ denote the symmetric group on $[n]$. For $\sigma \in \mathfrak{S}(n)$, we denote by

$$
\begin{aligned}
\operatorname{ver}(\sigma) & :=\left\{(a, b) \in[n]^{2} \mid a \leqslant b \text { and } \sigma^{-1}(a) \leqslant \sigma^{-1}(b)\right\} \\
\text { and } \quad \operatorname{inv}(\sigma) & :=\left\{(b, a) \in[n]^{2} \mid a \leqslant b \text { and } \sigma^{-1}(a) \geqslant \sigma^{-1}(b)\right\}
\end{aligned}
$$

the set of versions and inversions of $\sigma$ respectively ${ }^{(2)}$. Inversions are classical (although we order their entries in a strange way), while versions are borrowed from [6]. Clearly, the versions of $\sigma$ determine the inversions of $\sigma$ and vice versa. The weak order on $\mathfrak{S}(n)$

\footnotetext{
${ }^{(1)}$ A comment on the notations used along this section. We use different notations for the set of permutations $\mathfrak{S}(n)$ and the set of corresponding posets WOEP. Although it might look like a complicated notation for a well-known object, we want our notation to clearly distinguish between the combinatorial objects and their corresponding posets.

${ }^{(2)}$ Throughout the paper, we only work with versions and inversions of values (sometimes called left inversions, or coinversions). The cover relations of the weak order are thus given by transpositions of consecutive positions (sometimes called right weak order). As there is no ambiguity in the paper, we never specify this convention.
} 


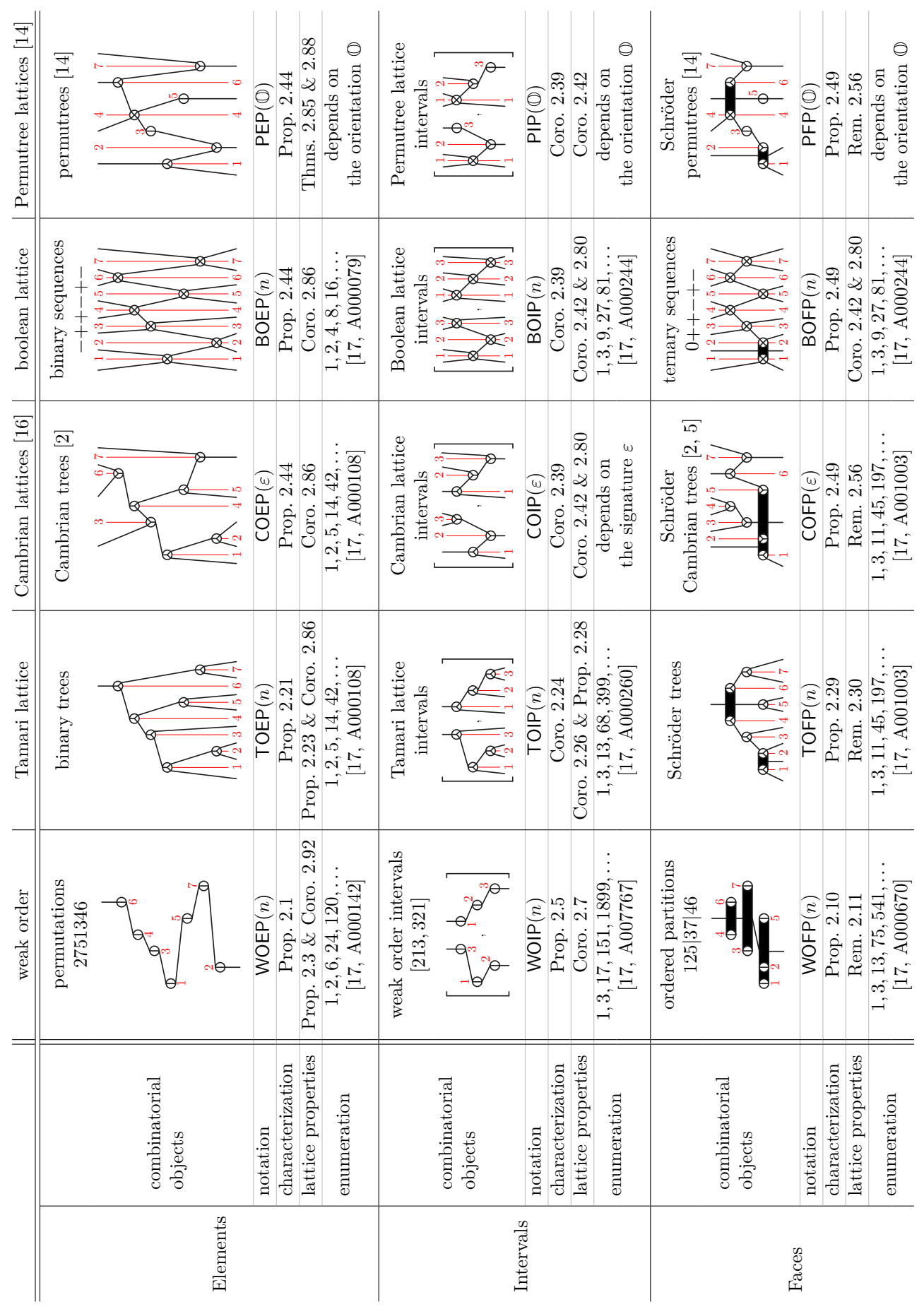

TABLE 1. A roadmap through the combinatorial objects considered in Section 2. 


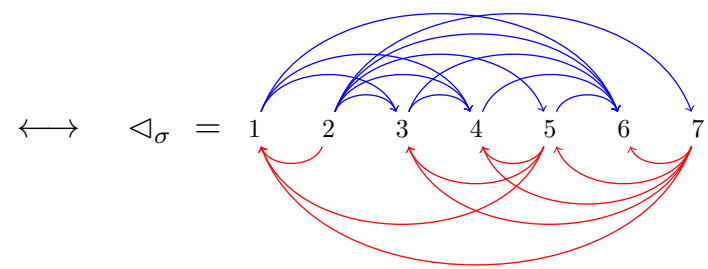

$$
\begin{aligned}
& \operatorname{ver}(\sigma)=\{(1,3),(1,4), \ldots,(5,6)\} \quad \longleftrightarrow \quad \triangleleft_{\sigma}^{\operatorname{lnc}}=1 \\
& \operatorname{inv}(\sigma)=\{(2,1),(7,5), \ldots,(5,4)\} \quad \longleftrightarrow \quad \triangleleft_{\sigma}^{\text {Dec }}=1
\end{aligned}
$$
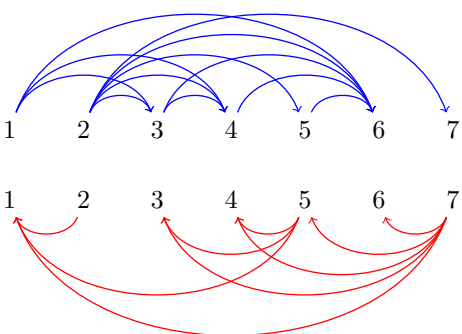

Figure 6. A Weak Order Element Poset (WOEP).

is defined as the inclusion order of inversions, or as the clusion (reverse inclusion) order of the versions:

$$
\sigma \preccurlyeq \tau \Longleftrightarrow \operatorname{inv}(\sigma) \subseteq \operatorname{inv}(\tau) \Longleftrightarrow \operatorname{ver}(\sigma) \supseteq \operatorname{ver}(\tau) .
$$

It is known that the weak order on $\mathfrak{S}(n)$ is a lattice. We denote by $\wedge_{\mathfrak{S}}$ and $\vee_{\mathfrak{S}}$ its meet and join, and by $e:=[1,2, \ldots, n]$ and $w_{\circ}:=[n, \ldots, 2,1]$ the weak order minimal and maximal permutations.

2.1.1. Weak Order Element Posets. We see a permutation $\sigma \in \mathfrak{S}(n)$ as a total order $\triangleleft_{\sigma}$ on $[n]$ defined by $u \triangleleft_{\sigma} v$ if $\sigma^{-1}(u) \leqslant \sigma^{-1}(v)$ (i.e. $u$ is before $v$ in $\sigma$ ). In other words, $\triangleleft_{\sigma}$ is the chain $\sigma(1) \triangleleft_{\sigma} \ldots \triangleleft_{\sigma} \sigma(n)$ as illustrated in Figure 6 .

We say that $\triangleleft_{\sigma}$ is a weak order element poset, and we denote by

$$
\operatorname{WOEP}(n):=\left\{\triangleleft_{\sigma} \mid \sigma \in \mathfrak{S}(n)\right\}
$$

the set of all total orders on $[n]$. The following characterization of these elements is immediate.

Proposition 2.1. A poset $\triangleleft \in \mathcal{P}(n)$ is in $\operatorname{WOEP}(n)$ if and only if $\forall u, v \in[n]$, either $u \triangleleft v$ or $u \triangleright v$.

In other words, the WOEP are the maximal posets, with $\left(\begin{array}{c}n \\ 2\end{array}\right)$ relations (this should help spotting them on Figure 5). The following proposition connects the weak order on $\mathfrak{S}(n)$ to that on $\mathcal{P}(n)$. It justifies the term "weak order" used in Definition 1.1.

Proposition 2.2. For $\sigma \in \mathfrak{S}(n)$, the increasing (resp. decreasing) relations of $\triangleleft_{\sigma}$ are the versions (resp. inversions) of $\sigma: \triangleleft_{\sigma}^{\operatorname{lnc}}=\operatorname{ver}(\sigma)$ and $\triangleleft_{\sigma}^{\operatorname{Dec}}=\operatorname{inv}(\sigma)$. Therefore, for any permutations $\sigma, \sigma^{\prime} \in \mathfrak{S}(n)$, we have $\sigma \preccurlyeq \sigma^{\prime}$ if and only if $\triangleleft_{\sigma} \preccurlyeq \triangleleft_{\sigma^{\prime}}$.

Proof. By definitions, we have

$$
\begin{aligned}
\triangleleft_{\sigma}^{\operatorname{lnc}} & =\left\{(a, b) \mid a<b \text { and } a \triangleleft_{\sigma} b\right\} \\
& =\left\{(a, b) \mid a<b \text { and } \sigma^{-1}(a)<\sigma^{-1}(b)\right\}=\operatorname{ver}(\sigma) .
\end{aligned}
$$

We thus obtain that the subposet of the weak order on $\mathcal{P}(n)$ induced by the set $\operatorname{WOEP}(n)$ is isomorphic to the weak order on $\mathfrak{S}(n)$, and thus is a lattice. To 


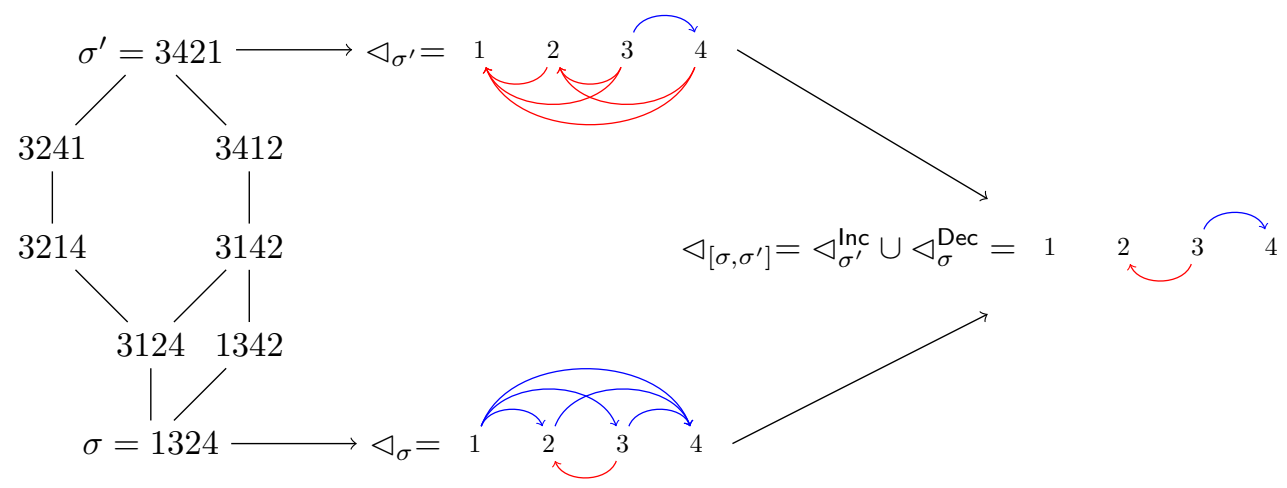

Figure 7. A Weak Order Interval Poset (WOIP).

conclude on $\operatorname{WOEP}(n)$, we mention the following stronger statement which will be derived in Corollary 2.92 .

Proposition 2.3. The set $\operatorname{WOEP}(n)$ induces a sublattice of the weak order on $\mathcal{P}(n)$.

2.1.2. Weak Order Interval Posets. For two permutations $\sigma, \sigma^{\prime} \in \mathfrak{S}(n)$ with $\sigma \preccurlyeq \sigma^{\prime}$, we denote by $\left[\sigma, \sigma^{\prime}\right]:=\left\{\tau \in \mathfrak{S}(n) \mid \sigma \preccurlyeq \tau \preccurlyeq \sigma^{\prime}\right\}$ the weak order interval between $\sigma$ and $\sigma^{\prime}$. As illustrated in Figure 7, we can see such an interval as the set of linear extensions of a poset.

Proposition 2.4. The permutations of $\left[\sigma, \sigma^{\prime}\right]$ are precisely the linear extensions of the poset

$$
\triangleleft_{\left[\sigma, \sigma^{\prime}\right]}:=\bigcap_{\sigma \preccurlyeq \tau \preccurlyeq \sigma^{\prime}} \triangleleft_{\tau}=\triangleleft_{\sigma} \cap \triangleleft_{\sigma^{\prime}}=\triangleleft_{\sigma^{\prime}}^{\operatorname{lnc}} \cup \triangleleft_{\sigma}^{\text {Dec }} .
$$

Proof. We first prove that the three expressions for $\triangleleft_{\left[\sigma, \sigma^{\prime}\right]}$ coincide. Indeed we have

$$
\bigcap_{\sigma \preccurlyeq \tau \preccurlyeq \sigma^{\prime}} \triangleleft_{\tau}=\left(\bigcap_{\sigma \preccurlyeq \tau \preccurlyeq \sigma^{\prime}} \triangleleft_{\tau}^{\text {Inc }}\right) \cup\left(\bigcap_{\sigma \preccurlyeq \tau \preccurlyeq \sigma^{\prime}} \triangleleft_{\tau}^{\text {Dec }}\right)=\triangleleft_{\sigma^{\prime}}^{\operatorname{lnc}} \cup \triangleleft_{\sigma}^{\text {Dec }}=\triangleleft_{\sigma} \cap \triangleleft_{\sigma^{\prime}},
$$

where the first equality is obtained by restriction to the increasing and decreasing relations, the second equality holds since $\sigma \preccurlyeq \tau \preccurlyeq \sigma^{\prime} \Longleftrightarrow \triangleleft_{\tau}^{\text {Inc }} \supseteq \triangleleft_{\sigma^{\prime}}^{\text {Inc }}$ and $\triangleleft_{\sigma}^{\text {Dec }} \subseteq \triangleleft_{\tau}^{\text {Dec }}$ by Proposition 2.2, and the last one follows from $\triangleleft_{\sigma}^{\operatorname{lnc}} \supseteq \triangleleft_{\sigma^{\prime}}^{\operatorname{lnc}}$ and $\triangleleft_{\sigma}^{\text {Dec }} \subseteq \triangleleft_{\sigma^{\prime}}^{\text {Dec }}$.

Consider now a permutation $\tau$. By definition, $\triangleleft_{\tau}$ extends $\triangleleft_{\sigma^{\prime}}^{\text {Inc }} \cup \triangleleft_{\sigma}^{\text {Dec }}$ if and only if $\triangleleft_{\tau}^{\operatorname{Inc}} \supseteq \triangleleft_{\sigma^{\prime}}^{\operatorname{Inc}}$ and $\triangleleft_{\sigma}^{\text {Dec }} \subseteq \triangleleft_{\tau}^{\text {Dec }}$, which in turns is equivalent to $\sigma \preccurlyeq \tau \preccurlyeq \sigma^{\prime}$ by Proposition 2.2.

We say that $\triangleleft_{\left[\sigma, \sigma^{\prime}\right]}$ is a weak order interval poset, and we denote by

$$
\operatorname{WOIP}(n):=\left\{\triangleleft_{\left[\sigma, \sigma^{\prime}\right]} \mid \sigma, \sigma^{\prime} \in \mathfrak{S}(n), \sigma \preccurlyeq \sigma^{\prime}\right\}
$$

the set of all weak order interval posets on $[n]$. The following characterization of these posets already appeared in [1, Thm. 6.8] and will be discussed in Section 2.1.4.

Proposition 2.5 ([1, Thm. 6.8]). A poset $\triangleleft \in \mathcal{P}(n)$ is in $\operatorname{WOIP}(n)$ if and only if $\forall a<b<c$,

$$
a \triangleleft c \Longrightarrow a \triangleleft b \text { or } b \triangleleft c \quad \text { and } \quad a \triangleright c \Longrightarrow a \triangleright b \text { or } b \triangleright c .
$$

We now describe the weak order on $\operatorname{WOIP}(n)$.

Proposition 2.6. For any $\sigma \preccurlyeq \sigma^{\prime}$ and $\tau \preccurlyeq \tau^{\prime}$, we have $\triangleleft_{\left[\sigma, \sigma^{\prime}\right]} \preccurlyeq \triangleleft_{\left[\tau, \tau^{\prime}\right]}$ if and only if $\sigma \preccurlyeq \tau$ and $\sigma^{\prime} \preccurlyeq \tau^{\prime}$. 


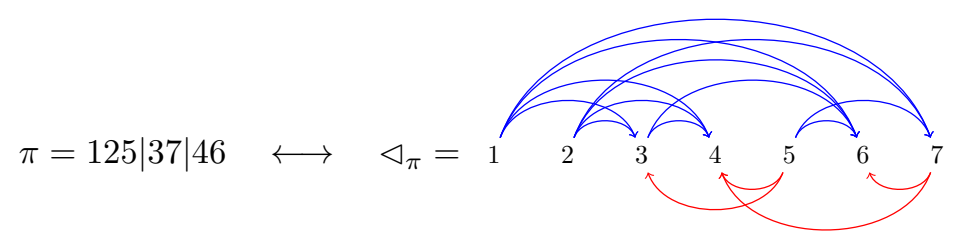

Figure 8. A Weak Order Face Poset (WOFP).

Proof. From the formula of Proposition 2.4, we have

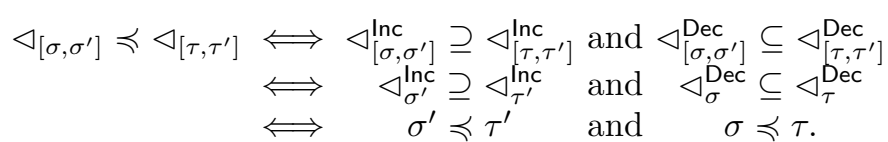

It follows that the weak order on $\operatorname{WOIP}(n)$ gets the lattice structure of a product, described in the next statement. See also Corollary 2.20 for an alternative description of the meet and join in this lattice.

COROLlaRY 2.7. The weak order on $\operatorname{WOIP}(n)$ is a lattice whose meet and join are given by

$$
\begin{aligned}
& \triangleleft_{\left[\sigma, \sigma^{\prime}\right]} \wedge_{\mathrm{WOIP}} \triangleleft_{\left[\tau, \tau^{\prime}\right]}=\triangleleft_{\left[\sigma \wedge_{\mathfrak{S}} \tau, \sigma^{\prime} \wedge_{\mathfrak{S}} \tau^{\prime}\right]} \\
& \text { and } \triangleleft_{\left[\sigma, \sigma^{\prime}\right]} \vee_{\text {WOIP }} \triangleleft_{\left[\tau, \tau^{\prime}\right]}=\triangleleft_{\left[\sigma \vee_{\mathfrak{S}} \tau, \sigma^{\prime} \vee_{\mathfrak{S}} \tau^{\prime}\right]} \text {. }
\end{aligned}
$$

COROLlary 2.8. The set $\operatorname{WOEP}(n)$ induces a sublattice of the weak order on WOIP.

REMARK 2.9. The weak order on $\operatorname{WOIP}(n)$ is not a sublattice of the weak order on $\mathcal{P}(n)$. For example,

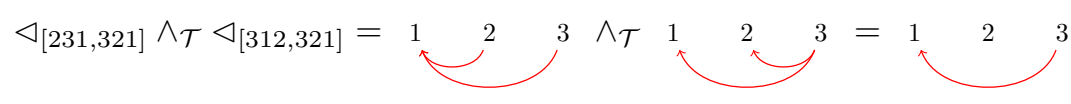

while $\triangleleft_{[231,321]} \wedge_{\text {WOIP }} \triangleleft_{[312,321]}=\triangleleft_{[123,321]}=\varnothing($ trivial poset on [3] $)$.

2.1.3. Weak Order Face Posets. The permutations of $\mathfrak{S}(n)$ correspond to the vertices of the permutahedron $\operatorname{Perm}(n):=\operatorname{conv}\{(\sigma(1), \ldots, \sigma(n)) \mid \sigma \in \mathfrak{S}(n)\}$. We now consider all the faces of the permutahedron. The codimension $k$ faces of $\operatorname{Perm}(n)$ correspond to ordered partitions of $[n]$ into $k$ parts, or equivalently to surjections from $[n]$ to $[k]$. We see an ordered partition $\pi$ as a poset $\triangleleft_{\pi}$ on $[n]$ defined by $u \triangleleft_{\pi} v$ if and only if $u=v$ or $\pi^{-1}(u)<\pi^{-1}(v)$, that is, the part of $\pi$ containing $u$ appears strictly before the part of $\pi$ containing $v$. See Figure 8 . Note that a permutation $\sigma$ belongs to the face of the permutahedron $\operatorname{Perm}(n)$ corresponding to an ordered partition $\pi$ if and only if $\triangleleft_{\sigma}$ is a linear extension of $\triangleleft_{\pi}$.

We say that $\triangleleft_{\pi}$ is a weak order face poset, and we denote by

$$
\operatorname{WOFP}(n):=\left\{\triangleleft_{\pi} \mid \pi \text { ordered partition of }[n]\right\}
$$

the set of all weak order face posets on $[n]$. We first characterize these posets.

Proposition 2.10. The following conditions are equivalent for a poset $\triangleleft \in \mathcal{P}(n)$ :

(1) $\triangleleft \in \operatorname{WOFP}(n)$,

(2) $\forall u, v, w \in[n], \quad u \triangleleft w \Longrightarrow u \triangleleft v$ or $v \triangleleft w$,

(3) $\triangleleft \in \operatorname{WOIP}(n)$ and $\forall a<b<c$ with a, $c$ incomparable, $a \triangleleft b \Longleftrightarrow b \triangleright c$ and $a \triangleright b \Longleftrightarrow b \triangleleft c$.

Proof. Assume that $\triangleleft=\triangleleft_{\pi} \in \operatorname{WOFP}(n)$ for an ordered partition $\pi$ of $[n]$, and let $u, v, w \in[n]$ such that $u \triangleleft w$. By definition, we have $\pi^{-1}(u)<\pi^{-1}(w)$. Therefore, 
we certainly have $\pi^{-1}(u)<\pi^{-1}(v)$ or $\pi^{-1}(v)<\pi^{-1}(w)$, and thus $u \triangleleft v$ or $v \triangleleft w$. This proves that $(1) \Longrightarrow(2)$.

Assume now that $\triangleleft$ satisfies (2). It immediately implies that $\triangleleft \in \operatorname{WOIP}(n)$ by the characterization of Proposition 2.5. Consider now $a<b<c$ such that $a$ and $c$ are incomparable in $\triangleleft$. If $a \triangleleft b$, then (2) implies that either $a \triangleleft c$ or $c \triangleleft b$. Since we assumed that $a$ and $c$ are incomparable, we obtain that $b \triangleright c$. We obtain similarly that $b \triangleright c \Longrightarrow a \triangleleft b$, that $a \triangleright b \Longrightarrow b \triangleleft c$ and that $b \triangleleft c \Longrightarrow a \triangleright b$. This shows that $(2) \Longrightarrow(3)$.

Finally, assume that $\triangleleft$ satisfies (3). Consider the $\triangleleft$ incomparability relation $\equiv$ defined by $u \equiv v$ when $u$ and $v$ are incomparable in $\triangleleft$. Condition (3) ensures that $\equiv$ is an equivalence relation. Moreover, the equivalence classes of $\equiv$ are totally ordered. This shows that $\triangleleft$ defines an ordered partition of $[n]$ and thus that $(3) \Longrightarrow(1)$.

We now consider the weak order on $\operatorname{WOFP}(n)$. Since $\operatorname{WOFP}(n) \subseteq \operatorname{WOIP}(n)$, Proposition 2.6 shows that we have $\triangleleft \preccurlyeq \triangleleft \Longleftrightarrow \triangleleft^{\text {minle }} \preccurlyeq \triangleleft^{\text {minle }}$ and $\triangleleft^{\text {maxle }} \preccurlyeq \triangleleft^{\text {maxle }}$. This order is precisely the facial weak order on the permutahedron $\operatorname{Perm}(n)$ studied by A. Dermenjian, C. Hohlweg and V. Pilaud in [4]. They prove in particular that this order coincides with the pseudo-permutahedron originally defined by D. Krob, M. Latapy, J.-C. Novelli, H.-D. Phan and S. Schwer [7] on ordered partitions as the transitive closure of the relations

$$
\lambda_{1}|\cdots| \lambda_{i}\left|\lambda_{i+1}\right| \cdots\left|\lambda_{k} \prec \lambda_{1}\right| \cdots\left|\lambda_{i} \lambda_{i+1}\right| \cdots\left|\lambda_{k} \prec \lambda_{1}\right| \cdots\left|\lambda_{i+1}\right| \lambda_{i}|\cdots| \lambda_{k},
$$

if $\max \left(\lambda_{i}\right)<\min \left(\lambda_{i+1}\right)$. This order is known to be a lattice $[4,7]$. We will discuss an alternative description of the meet and join in this lattice in Section 2.4.4.

REMARK 2.11. Note that the weak order on $\operatorname{WOFP}(n)$ is not a sublattice of the weak order on $\mathcal{P}(n)$, nor on $\operatorname{WOIP}(n)$. For example,

$$
\begin{aligned}
& \triangleleft_{2 \mid 13} \wedge_{\mathcal{T}} \triangleleft_{123}=\triangleleft_{2 \mid 13} \wedge_{\text {WOIP }} \triangleleft_{123}=\{(2,3)\} \\
\text { while } & \triangleleft_{2 \mid 13} \wedge_{\text {WOFP }} \triangleleft_{123}=\triangleleft_{12 \mid 3}=\{(1,3),(2,3)\} .
\end{aligned}
$$

2.1.4. IWOIP $(n)$ and $\operatorname{DWOIP}(n)$ and the WOIP deletion. We conclude our section on the permutahedron by introducing some variations on $\operatorname{WOIP}(n)$ which are needed later and provide a proof of the characterization of $\operatorname{WOIP}(n)$ given in Proposition 2.5.

Since the set of linear extensions of a poset is order-convex, a poset is in $\operatorname{WOIP}(n)$ if and only if it admits weak order minimal and maximal linear extensions. This motivates to consider separately two bigger families of posets. Denote by $\operatorname{IWOIP}(n)$ (resp. by DWOIP $(n)$ ) the set of posets of $\mathcal{P}(n)$ which admit a weak order maximal (resp. minimal) linear extension. Proposition 2.5 follows from the characterization of these posets, illustrated in Figure 9.

Proposition 2.12. For a poset $\triangleleft \in \mathcal{P}(n)$,

$$
\begin{aligned}
& \triangleleft \in \operatorname{IWOIP}(n) \quad \Longleftrightarrow \quad \forall a<b<c, a \triangleleft c \Longrightarrow a \triangleleft b \text { or } b \triangleleft c, \\
& \triangleleft \in \operatorname{DWOIP}(n) \quad \Longleftrightarrow \quad \forall a<b<c, a \triangleright c \Longrightarrow a \triangleright b \text { or } b \triangleright c .
\end{aligned}
$$

Proof. By symmetry, we only prove the characterization of $\operatorname{IWOIP}(n)$. Assume first that $\triangleleft \in \mathcal{P}(n)$ is such that $a \triangleleft c \Longrightarrow a \triangleleft b$ or $b \triangleleft c$ for all $a<b<c$. Let

$$
\triangleleft^{\text {maxle }}:=\triangleleft \cup\{(b, a) \mid a<b \text { incomparable in } \triangleleft\}
$$

denote the binary relation obtained from $\triangleleft$ by adding a decreasing relation between any two incomparable elements in $\triangleleft$ (see Figure 9 ). The following claim is proved in Appendix A.1.

Claim 2.13. $\triangleleft^{\text {maxle }}$ is a poset. 
Moreover $\triangleleft^{\text {maxle }}$ is a total order (since any two elements are comparable in $\triangleleft^{\text {maxle }}$ by definition) which is a linear extension of $\triangleleft$ (since $\triangleleft \subseteq \triangleleft^{\text {maxle }}$ by definition). Finally, any other linear extension of $\triangleleft$ is smaller than $\triangleleft^{\text {maxle }}$ in weak order (since a linear extension of $\triangleleft$ contains $\triangleleft$ and $\triangleleft^{\text {maxle }} \backslash \triangleleft \subseteq \mathrm{D}_{n}$ ). We conclude that $\triangleleft^{\text {maxle }}$ is the maximal linear extension of $\triangleleft$ in weak order.

Reciprocally, assume now that there exists $a<b<c$ such that $a \triangleleft c$ while $a \nless b$ and $b \Varangle c$. The transitivity of $\triangleleft$ implies that $b \Varangle a$ and $c \nless b$. Let $\sim:=\triangleleft \cup\{(a, b),(c, b)\}$ and $\backsim:=\triangleleft \cup\{(b, a),(b, c)\}$. Note that $\sim$ and $\sim$ are still acyclic (but not necessary transitive). Indeed any cycle for example in $\sim$ would involve either $(a, b)$ or $(c, b)$, but not both. If $\sim$ has a cycle involving for example $(a, b)$, then $b \triangleleft a$ by transitivity of $\triangleleft$, which gives a contradiction. Thus they admit linear extensions, and we consider minimal linear extensions $\rho$ of $\sim$ and $\sigma$ of $\backsim$. We conclude that $\rho$ and $\sigma$ are minimal linear extensions of $\triangleleft$ incomparable in the weak order as illustrated on Figure 9 .

REMARK 2.14. Note that it is enough to check the conditions of Proposition 2.12 only for all cover relations $a \triangleleft c$ and $a \triangleright c$ of $\triangleleft$. Indeed, consider $a<b<c$ where $a \triangleleft c$ is not a cover relation, so that there exists $u \in[n]$ such that $a \triangleleft u \triangleleft c$. Assume for example that $b<u$, the case $u<b$ being symmetric. Hence $a<b<u$ and $a \triangleleft u$ implies that either $a \triangleleft b$ or $b \triangleleft u$ (by induction on the length of the minimal chain between $a$ and $c$ ). If $b \triangleleft u$, we obtain that $b \triangleleft u \triangleleft c$ so that $b \triangleleft c$.

We have seen in Corollary 2.7 that the weak order on $\operatorname{WOIP}(n)$ on interval posets forms a lattice. Using the characterization of Proposition 2.12, we now show that the subposets of the weak order on $\mathcal{P}(n)$ induced by the sets $\operatorname{IWOIP}(n)$ and $\operatorname{DWOIP}(n)$ form lattices - although there are not sublattices of the weak order on $\mathcal{P}(n)$. We define the IWOIP increasing deletion, the DWOIP decreasing deletion, and the WOIP
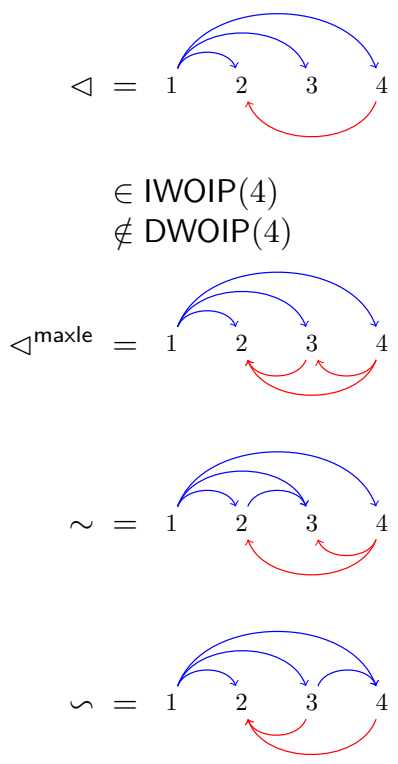

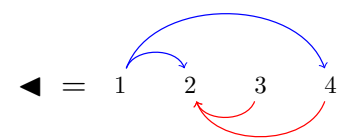

$$
\notin \operatorname{IWOIP}(4)
$$$$
\in \operatorname{DWOIP}(4)
$$
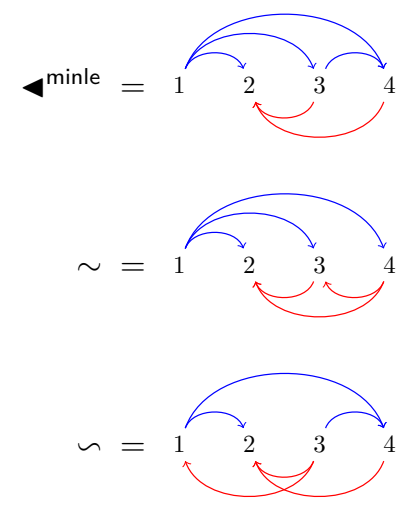

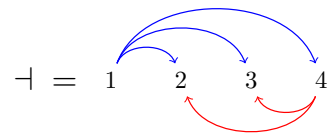

$\in \operatorname{WOIP}(4)$
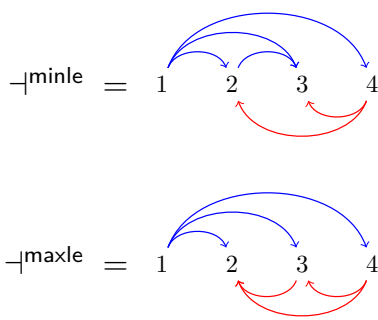

Figure 9. Examples and counterexamples of elements in IWOIP(4) and DWOIP(4). 


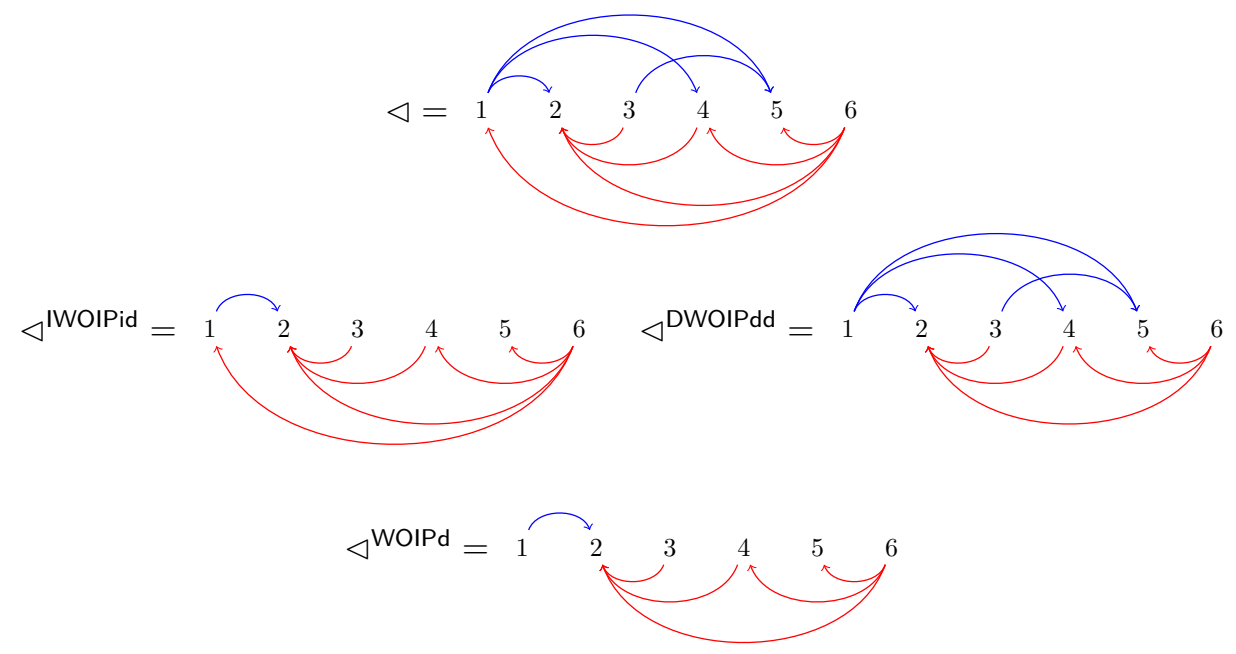

FiguRE 10. The IWOIP increasing deletion, the DWOIP decreasing deletion, and the WOIP deletion.

deletion by

$$
\begin{aligned}
\triangleleft^{\mathrm{IWOIPid}} & =\triangleleft \backslash\left\{(a, c) \mid \exists a<b_{1}<\cdots<b_{k}<c, a \not b_{1} \not \cdots \not b_{k} \not c\right\}, \\
\triangleleft^{\text {DWOIPdd }} & =\triangleleft \backslash\left\{(c, a) \mid \exists a<b_{1}<\cdots<b_{k}<c, a \not b_{1} \not \cdots \not b_{k} \not c\right\}, \\
\triangleleft^{\text {WOIPd }}: & =\left(\triangleleft^{\text {DWOIPdd }}\right)^{\text {IWOIPid }}=\left(\triangleleft^{\text {IWOIPid }}\right)^{\text {DWOIPdd }} .
\end{aligned}
$$

These operations are illustrated on Figure 10.

REMARK 2.15. Similar to Remark 1.10, the IWOIP increasing deletion (resp. DWOIP decreasing deletion) deletes at once all increasing relations which prevent the poset to be in $\operatorname{IWOIP}(n)$ (resp. in $\operatorname{DWOIP}(n)$ ). Deleting only the relations $(a, c)$ (resp. $(c, a))$ for which there exists $a<b<c$ such that $a \notin b \nless c$ (resp. $a \not b \not c)$ would require several iterations. For example, we would need $n$ iterations to obtain $\{(i, j) \mid i, j \in[n], i+1<j\}^{\text {IWOIPid }}=\varnothing$.

These functions satisfy the following properties.

LEMMA 2.16. For any poset $\triangleleft \in \mathcal{P}(n)$, we have $\triangleleft^{\operatorname{IWOIPid}} \in \operatorname{IWOIP}(n)$ and $\triangleleft^{\mathrm{DWOIPdd}} \in \operatorname{DWOIP}(n)$. Moreover, $\triangleleft \in \operatorname{DWOIP}(n) \Longrightarrow \triangleleft^{\operatorname{IWOIPid}} \in \operatorname{WOIP}(n)$ and $\triangleleft \in \operatorname{IWOIP}(n) \Longrightarrow \triangleleft^{\mathrm{DWOIPdd}} \in \operatorname{WOIP}(n)$.

Proof. We prove the result for $\triangleleft^{\text {IWOIPid }}$, the proof for $\triangleleft^{\text {DWOIPdd }}$ being symmetric. The details of the following claim are given in Appendix A.1.

Claim 2.17. $\triangleleft^{\text {IWOIPid }}$ is a poset.

Thus the characterization of Proposition 2.12 implies that $\triangleleft^{\text {IWOIPid }}$ is always in $\operatorname{IWOIP}(n)$, and even in $\operatorname{WOIP}(n)$ when $\triangleleft \in \operatorname{DWOIP}(n)$.

LEMMA 2.18. For any poset $\triangleleft \in \mathcal{P}(n)$, the poset $\triangleleft^{\text {IWOIPid }}$ (resp. $\triangleleft^{\text {DWOIPdd }}$ ) is the weak order minimal (resp. maximal) poset in $\operatorname{IWOIP}(n)$ bigger than $\triangleleft$ (resp. in $\operatorname{DWOIP}(n)$ smaller than $\triangleleft)$.

Proof. We prove the result for $\triangleleft^{\text {IWOIPid }}$, the proof for $\triangleleft^{\text {DWOIPdd }}$ being symmetric. Observe first that $\triangleleft \preccurlyeq \triangleleft^{\text {IWOIPid }}$ since $\triangleleft^{\text {IWOIPid }}$ is obtained from $\triangleleft$ by deleting increasing relations. Consider now $\triangleleft \in \operatorname{IWOIP}(n)$ such that $\triangleleft \preccurlyeq \varangle$. By definition, 


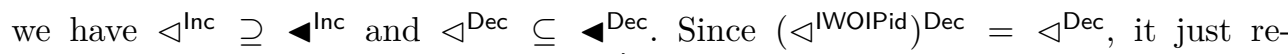
mains to show that for any $(a, c) \in \varangle^{\text {Inc }}$, there exist no $a<b_{1}<\cdots<b_{k}<c$ with $a \Varangle b_{1} \Varangle b_{2} \Varangle \cdots \Varangle b_{k} \Varangle c$. Assume otherwise and choose such a pair $(a, c)$ with $c-a$ minimal. Since $4 \in \operatorname{IWOIP}(n)$ and $a<b_{1}<c$ are such that $a<c$ while $a b_{1}$ (because $a \Varangle^{\text {Inc }} b_{1}$ and $\varangle^{\text {Inc }} \subset \triangleleft^{\operatorname{lnc}}$ ), we have $b_{1} \triangleleft c$. But this assertion contradicts the minimality of $c-a$.

Proposition 2.19. The subposets of the weak order on $\mathcal{P}(n)$ induced by $\operatorname{IWOIP}(n)$ and $\operatorname{DWOIP}(n)$ are lattices whose meets and joins are given by

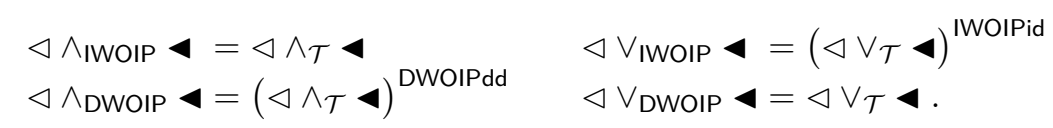

Proof. We prove the result for $\operatorname{IWOIP}(n)$, the proof for $\operatorname{DWOIP}(n)$ being symmetric. Consider $\triangleleft, \varangle \in \operatorname{IWOIP}(n)$. We first prove that $\dashv:=\triangleleft \wedge_{\mathcal{T}} \triangleleft=\left(\left(\triangleleft^{\text {lnc }} \cup \triangleleft^{\text {Inc }}\right)^{\text {tc }} \cup\right.$ $\left.\left(\triangleleft^{\mathrm{Dec}} \cap \triangleleft^{\mathrm{Dec}}\right)\right)^{\text {tdd }}$ is also in IWOIP $(n)$ (see also Proposition 2.73 and Example 2.74 for a more systematic approach). For any cover relation $a \dashv c$ and $a<b<c$, we have $a \dashv^{\text {Inc }} c$ so that $a \triangleleft^{\operatorname{lnc}} c$ or $a \varangle^{\operatorname{lnc}} c$ (since we have a cover relation). Since $\triangleleft, \triangleleft \in \operatorname{IWOIP}(n)$, we obtain that $a \triangleleft^{\ln c} b$, or $b \triangleleft^{\operatorname{lnc}} c$, or $a \varangle^{\ln c} b$, or $b \triangleleft^{\operatorname{lnc}} c$. Thus, $a \dashv b$ or $b \dashv c$ for any cover relation $a \dashv c$ and any $a<b<c$. Using Remark 2.14, we conclude that $\dashv \in \operatorname{IWOIP}(n)$.

On the other hand, Lemma 2.18 asserts that $\left(\triangleleft \vee_{\mathcal{T}} \triangleleft\right)^{\text {IWOIPid }}$ is the weak order minimal poset in $\operatorname{IWOIP}(n)$ bigger than $\triangleleft \vee_{\mathcal{T}} \triangleleft$. Any poset in $\operatorname{IWOIP}(n)$ bigger than $\triangleleft$ and $\varangle$ is also bigger than $\triangleleft \vee_{\mathcal{T}} \varangle$, and thus bigger than $\left(\triangleleft \vee_{\mathcal{T}} \triangleleft\right)^{\text {IWoIPid }}$. We conclude that $\left(\triangleleft \vee_{\mathcal{T}} \triangleleft\right)^{\text {IWOIPid }}$ is indeed the join of $\triangleleft$ and $\triangleleft$.

We finally deduce from Proposition 2.19 and Lemma 2.16 an alternative formula for the meet and join in the weak order on $\operatorname{WOIP}(n)$. See also Corollary 2.7.

COROLlary 2.20. The meet and join in the weak order on $\operatorname{WOIP}(n)$ are given by

$$
\triangleleft \wedge_{\text {WOIP }} \triangleleft=\left(\triangleleft \wedge_{\mathcal{T}} \triangleleft\right)^{\text {DWOIPdd }} \quad \text { and } \quad \triangleleft \vee_{\text {WOIP }} \triangleleft=\left(\triangleleft \vee_{\mathcal{T}} \triangleleft\right)^{\text {IWOIPid }} \text {. }
$$

2.2. From the ASSOCiAhedron. Similarly to the previous section, we now briefly discuss some relevant families of posets corresponding to the elements, the intervals, and the faces of the associahedron. Further similar families of posets arising from permutreehedra [14] will be discussed in Section 2.3. This section should just be considered as a simplified prototype to the next section. We therefore omit the proofs which will appear in a more general context in Sections 2.3 and 2.4.

We denote by $\mathfrak{B}(n)$ the set of planar rooted binary trees with $n$ nodes, that we simply call binary trees here for short. We label the vertices of a binary tree $\mathrm{T} \in \mathfrak{B}(n)$ through an inorder traversal, i.e. such that all vertices in the left (resp. right) child of a vertex $v$ of $\mathrm{T}$ receive a label smaller (resp. larger) than the label of $v$. From now on, we identify a vertex and its label.

There is a fundamental surjection from permutations to binary trees. Namely, a permutation $\sigma:=\sigma_{1} \ldots \sigma_{n} \in \mathfrak{S}(n)$ is mapped to the binary tree $\operatorname{bt}(\sigma) \in \mathfrak{B}(n)$ obtained by successive insertions of $\sigma_{n}, \ldots, \sigma_{1}$ in a binary (search) tree. The fiber of a tree $\mathrm{T}$ is precisely the set of linear extensions of $\mathrm{T}$. It is an interval of the weak order whose minimal and maximal elements respectively avoid the patterns 312 and 132 . Moreover, the fibers of bt define a lattice congruence of the weak order. Thus, the set $\mathfrak{B}(n)$ of binary trees is endowed with a lattice structure $\preccurlyeq$ defined by

$$
\mathrm{T} \preccurlyeq \mathrm{T}^{\prime} \Longleftrightarrow \exists \sigma, \sigma^{\prime} \in \mathfrak{S}(n) \text { such that } \operatorname{bt}(\sigma)=\mathrm{T} \text {, bt }\left(\sigma^{\prime}\right)=\mathrm{T}^{\prime} \text { and } \sigma \preccurlyeq \sigma^{\prime}
$$


whose meet $\wedge_{\mathfrak{B}}$ and join $\vee_{\mathfrak{B}}$ are given by

$$
\mathrm{T} \wedge_{\mathfrak{B}} \mathrm{T}^{\prime}=\operatorname{bt}\left(\sigma \wedge_{\mathfrak{S}} \sigma^{\prime}\right) \text { and } \mathrm{T} \vee_{\mathfrak{B}} \mathrm{T}^{\prime}=\operatorname{bt}\left(\sigma \vee_{\mathfrak{S}} \sigma^{\prime}\right)
$$

for any representatives $\sigma, \sigma^{\prime} \in \mathfrak{S}(n)$ such that $\operatorname{bt}(\sigma)=\mathrm{T}$ and $\operatorname{bt}\left(\sigma^{\prime}\right)=\mathrm{T}^{\prime}$. Note that in particular, $\mathrm{T} \preccurlyeq \mathrm{T}^{\prime}$ if and only if $\sigma \preccurlyeq \sigma^{\prime}$ where $\sigma$ and $\sigma^{\prime}$ denote the minimal (resp. maximal) linear extensions of $\mathrm{T}$ and $\mathrm{T}^{\prime}$ respectively. For example, the minimal (resp. maximal) tree is the left (resp. right) comb whose unique linear extension is $e:=[1,2, \ldots, n]$ (resp. $\left.w_{\circ}:=[n, \ldots, 2,1]\right)$. This lattice structure is the Tamari lattice whose cover relations are given by right rotations on binary trees. It was introduced by D. Tamari [10] on Dyck paths, our presentation is a more modern perspective $[1,16]$.

2.2.1. Tamari Order Element Posets. We consider the tree $\mathrm{T}$ as a poset $\triangleleft_{\mathrm{T}}$, defined by $i \triangleleft_{\mathrm{T}} j$ when $i$ is a descendant of $j$ in T. In other words, the Hasse diagram of $\triangleleft_{\mathrm{T}}$ is the tree $\mathrm{T}$ oriented towards its root. An illustration is provided in Figure 11. Note that the increasing (resp. decreasing) subposet of $\triangleleft_{\mathrm{T}}$ is given by $i \triangleleft_{\mathrm{T}}^{\operatorname{lnc}} j$ (resp. $i \triangleleft_{\mathrm{T}}^{\text {Dec }} j$ ) if and only if $i$ belongs to the left (resp. right) subtree of $j$ in T.

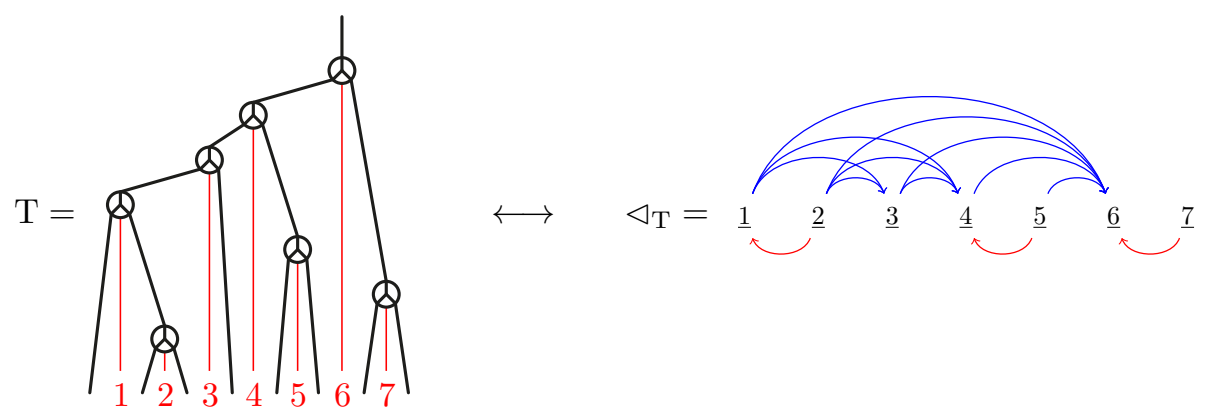

Figure 11. A Tamari Order Element Poset (TOEP).

We say that $\triangleleft_{\mathrm{T}}$ is a Tamari order element poset, and we denote by

$$
\operatorname{TOEP}(n):=\left\{\triangleleft_{\mathrm{T}} \mid \mathrm{T} \in \mathfrak{B}(n)\right\}
$$

the set of all Tamari order element posets on $[n]$. We first characterize them (see Proposition 2.44).

Proposition 2.21. A poset $\triangleleft \in \mathcal{P}(n)$ is in $\operatorname{TOEP}(n)$ if and only if

- $\forall a<b<c, a \triangleleft c \Longrightarrow b \triangleleft c$ and $a \triangleright c \Longrightarrow a \triangleright b$,

- for all $a<c$ incomparable in $\triangleleft$, there exists $a<b<c$ such that $a \triangleleft b \triangleright c$.

Now we establish the relationship between the Tamari lattice on $\mathfrak{B}(n)$ and the weak order on $\operatorname{TOEP}(n)$ (see Proposition 2.35).

Proposition 2.22. For any binary trees $\mathrm{T}, \mathrm{T}^{\prime} \in \mathfrak{B}(n)$, we have $\mathrm{T} \preccurlyeq \mathrm{T}^{\prime}$ in the Tamari lattice if and only if $\triangleleft_{\mathrm{T}} \preccurlyeq \triangleleft_{\mathrm{T}^{\prime}}$ in the weak order on posets.

It follows that the subposet of the weak order on $\mathcal{P}$ induced by the set $\operatorname{TOEP}(n)$ is isomorphic to the Tamari lattice on $\mathfrak{B}(n)$, and is thus a lattice. We conclude on $\operatorname{TOEP}(n)$ with the following stronger statement (see Theorem 2.85).

Proposition 2.23. The set $\operatorname{TOEP}(n)$ induces a sublattice of the weak order on $\mathcal{P}(n)$. 
2.2.2. Tamari Order Interval Posets. For two binary trees $\mathrm{T}, \mathrm{T}^{\prime} \in \mathfrak{B}(n)$ with $\mathrm{T} \preccurlyeq \mathrm{T}^{\prime}$, we denote by $\left[\mathrm{T}, \mathrm{T}^{\prime}\right]:=\left\{\mathrm{S} \in \mathfrak{B}(n) \mid \mathrm{T} \preccurlyeq \mathrm{S} \preccurlyeq \mathrm{T}^{\prime}\right\}$ the Tamari order interval between $\mathrm{T}$ and $\mathrm{T}^{\prime}$. We can see this interval as the poset

$$
\triangleleft_{\left[\mathrm{T}, \mathrm{T}^{\prime}\right]}:=\bigcap_{\mathrm{T} \preccurlyeq \mathrm{S} \preccurlyeq \mathrm{T}^{\prime}} \triangleleft_{\mathrm{T}}=\triangleleft_{\mathrm{T}} \cap \triangleleft_{\mathrm{T}^{\prime}}=\triangleleft_{\mathrm{T}^{\prime}}^{\text {Inc }} \cap \triangleleft_{\mathrm{T}}^{\text {Dec }} .
$$

See Figure 12 for an example.

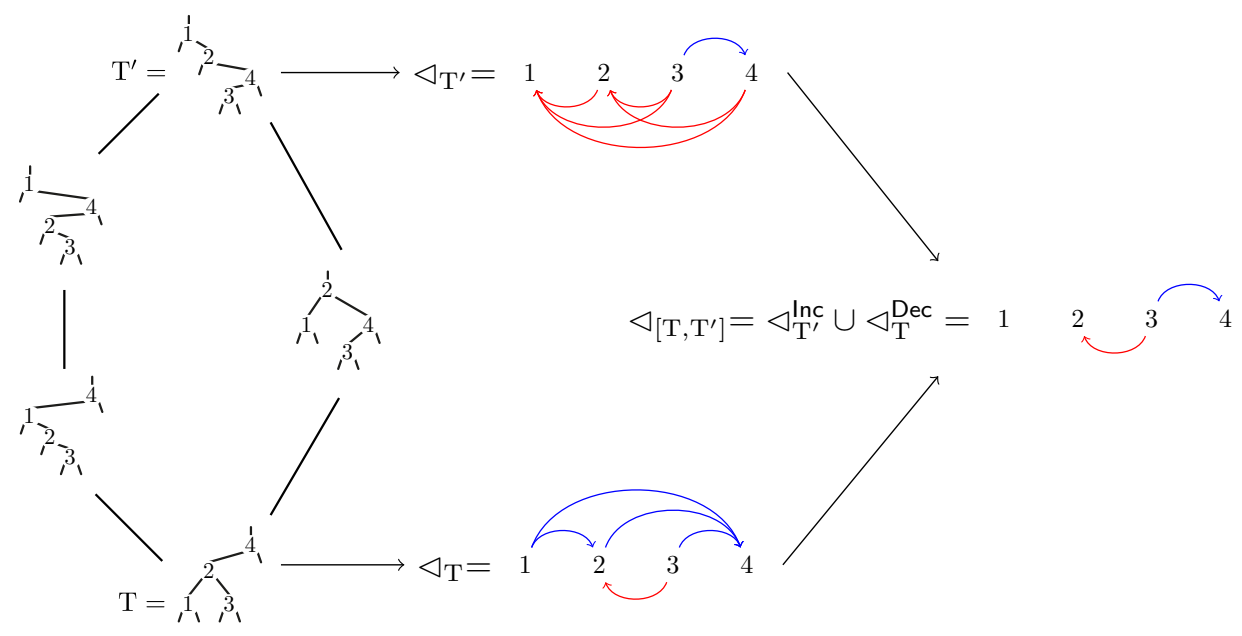

Figure 12. A Tamari Order Interval Poset (TOIP).

This poset $\triangleleft_{\left[\mathrm{T}, \mathrm{T}^{\prime}\right]}$ was introduced in [3] with the motivation that its linear extensions are precisely the linear extensions of all binary trees in the interval $\left[\mathrm{T}, \mathrm{T}^{\prime}\right]$. We say that $\triangleleft_{\left[\mathrm{T}, \mathrm{T}^{\prime}\right]}$ is a Tamari order interval poset, and we denote by

$$
\operatorname{TOIP}(n):=\left\{\triangleleft_{\left[\mathrm{T}, \mathrm{T}^{\prime}\right]} \mid \mathrm{T}, \mathrm{T}^{\prime} \in \mathfrak{B}(n), \mathrm{T} \preccurlyeq \mathrm{T}^{\prime}\right\}
$$

the set of all Tamari order interval posets on $[n]$. The following characterization of these posets (see Proposition 2.39) already appeared in [3, Thm. 2.8].

Corollary 2.24 ([3, Thm. 2.8]). A poset $\triangleleft \in \mathcal{P}(n)$ is in $\operatorname{TOIP}(n)$ if and only if $\forall a<b<c$,

$$
a \triangleleft c \Longrightarrow b \triangleleft c \quad \text { and } \quad a \triangleright c \Longrightarrow a \triangleright b .
$$

Now we describe the weak order on TOIP $(n)$ (see Proposition 2.41, Corollary 2.42). Proposition 2.25. For any $\mathrm{S} \preccurlyeq \mathrm{S}^{\prime}$ and $\mathrm{T} \preccurlyeq \mathrm{T}^{\prime}$, we have $\triangleleft_{\left[\mathrm{S}, \mathrm{S}^{\prime}\right]} \preccurlyeq \triangleleft_{\left[\mathrm{T}, \mathrm{T}^{\prime}\right]}$ if and only if $\mathrm{S} \preccurlyeq \mathrm{T}$ and $\mathrm{S}^{\prime} \preccurlyeq \mathrm{T}^{\prime}$.

COROllary 2.26. The weak order on $\operatorname{TOIP}(n)$ is a lattice whose meet and join are given by

$$
\begin{aligned}
\triangleleft_{\left[\mathrm{S}, \mathrm{S}^{\prime}\right]} \wedge_{\mathrm{TOIP}} \triangleleft_{\left[\mathrm{T}, \mathrm{T}^{\prime}\right]} & =\triangleleft_{\left[\mathrm{S} \wedge_{\mathfrak{B}} \mathrm{T}, \mathrm{S}^{\prime} \wedge_{\mathfrak{B}} \mathrm{T}^{\prime}\right]} \\
\text { and } \triangleleft_{\left[\mathrm{S}, \mathrm{S}^{\prime}\right]} \vee_{\mathrm{TOIP}} \triangleleft_{\left[\mathrm{T}, \mathrm{T}^{\prime}\right]} & =\triangleleft_{\left[\mathrm{S} \vee_{\mathfrak{B}} \mathrm{T}, \mathrm{S}^{\prime} \vee_{\mathfrak{B}} \mathrm{T}^{\prime}\right]} .
\end{aligned}
$$

Corollary 2.27. The set TOEP $(n)$ induces a sublattice of the weak order on TOIP.

In fact, we will derive the following statement (see Corollary 2.80).

Proposition 2.28. The set TOIP $(n)$ induces a sublattice of the weak order on $\mathcal{P}(n)$. 
2.2.3. Tamari Order Face Posets. The binary trees of $\mathfrak{B}(n)$ correspond to the vertices of the associahedron $\operatorname{Asso}(n)$ constructed e.g. by J.-L. Loday in [9]. We now consider all the faces of the associahedron $\operatorname{Asso}(n)$ which correspond to Schröder trees, i.e. planar rooted trees where each node has either none or at least two children. Given a Schröder tree S, we label the angles between consecutive children of the vertices of $\mathrm{S}$ in inorder, meaning that each angle is labeled after the angles in its left child and before the angles in its right child. Note that a binary tree $\mathrm{T}$ belongs to the face of the associahedron $\operatorname{Asso}(n)$ corresponding to a Schröder tree $\mathrm{S}$ if and only if $\mathrm{S}$ is obtained by edge contractions in $\mathrm{T}$. The set of such binary trees is an interval $\left[\mathrm{T}^{\min }(\mathrm{S}), \mathrm{T}^{\max }(\mathrm{S})\right]$ in the Tamari lattice, where the minimal (resp. maximal) tree $\mathrm{T}^{\mathrm{min}}(\mathrm{S})$ (resp. $\mathrm{T}^{\max }(\mathrm{S})$ ) is obtained by replacing the nodes of $\mathrm{S}$ by left (resp. right) combs as illustrated in Figure 13.

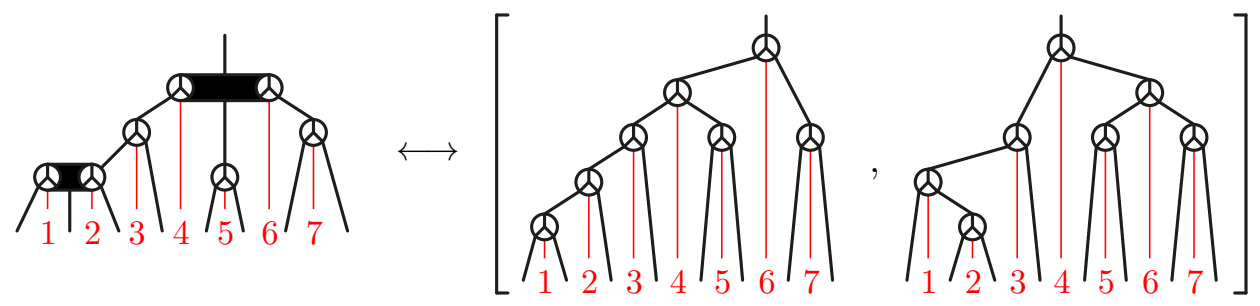

Figure 13. A Schröder tree $\mathrm{S}$ (left) can be seen as an interval $\left[\mathrm{T}^{\min }(\mathrm{S}), \mathrm{T}^{\max }(\mathrm{S})\right]$ in the Tamari lattice (right).

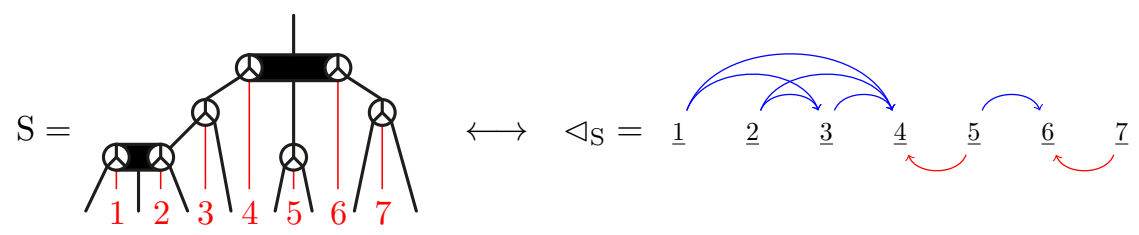

Figure 14. A Tamari Order Face Poset (TOFP).

We associate to a Schröder tree $\mathrm{S}$ the poset $\triangleleft_{\mathrm{S}}:=\triangleleft_{\left[\mathrm{T}^{\min }(\mathrm{S}), \mathrm{T}^{\max }(\mathrm{S})\right]}$. Equivalently, $i \triangleleft_{\mathrm{S}} j$ if and only if the angle $i$ belongs to the left or the right child of the angle $j$. See Figure 14. Note that

- a binary tree $\mathrm{T}$ belongs to the face of the associahedron $\operatorname{Asso}(n)$ corresponding to a Schröder tree $S$ if and only if $\triangleleft_{T}$ is an extension of $\triangleleft_{S}$, and

- the linear extensions of $\triangleleft_{\mathrm{S}}$ are precisely the linear extensions of $\triangleleft_{\mathrm{T}}$ for all binary trees $\mathrm{T}$ which belong to the face of the associahedron $\operatorname{Asso}(n)$ corresponding to $\mathrm{S}$.

We say that $\triangleleft_{\mathrm{S}}$ is a Tamari order face poset, and we denote by

$$
\operatorname{TOFP}(n):=\left\{\triangleleft_{\mathrm{S}} \mid \mathrm{S} \text { Schröder tree on }[n]\right\}
$$

the set of all Tamari order face posets. We first characterize these posets (see Proposition 2.49). 
Proposition 2.29. A poset $\triangleleft \in \mathcal{P}(n)$ is in $\operatorname{TOFP}(n)$ if and only if $\triangleleft \in \operatorname{TOIP}(n)$ (see characterization in Corollary 2.24) and for all $a<c$ incomparable in $\triangleleft$, either there exists $a<b<c$ such that $a \not b \Varangle c$, or for all $a<b<c$ we have $a \triangleright b \triangleleft c$.

Consider now the weak order on $\operatorname{TOFP}(n)$. It turns out (see Proposition 2.55) that this order on Schröder trees coincides with the facial weak order on the associahedron $\operatorname{Asso}(n)$ studied in $[4,11,12]$. This order is a quotient of the facial weak order on the permutahedron by the fibers of the Schröder tree insertion st. In particular, the weak order on $\operatorname{TOFP}(n)$ is a lattice.

REMARK 2.30. Remark 2.11 shows that the weak order on $\operatorname{TOFP}(n)$ is not a sublattice of the weak order on $\mathcal{P}(n)$, nor on $\operatorname{WOIP}(n)$, nor on $\operatorname{TOIP}(n)$.

2.2.4. TOIP deletion. We finally define a projection from all posets of $\mathcal{P}(n)$ to $\operatorname{TOIP}(n)$. We call TOIP deletion the map defined by

$$
\triangleleft^{\mathrm{TOIPd}}:=\triangleleft \backslash(\{(a, c) \mid \exists a<b<c, b \not c\} \cup\{(c, a) \mid \exists a<b<c, a \not b\}) .
$$

Then $\triangleleft^{\operatorname{TOIPd}} \in \operatorname{TOIP}(n)$ for any poset $\triangleleft \in \mathcal{P}(n)$ (see Lemma 2.58). We compare this map with the binary search tree and Schröder tree insertions described earlier (see Proposition 2.64, Corollary 2.67 and Proposition 2.68).

Proposition 2.31. For any permutation $\sigma \in \mathfrak{S}(n)$, any permutations $\sigma, \sigma^{\prime} \in \mathfrak{S}(n)$ with $\sigma \preccurlyeq \sigma^{\prime}$, and any ordered partition $\pi$ of $[n]$, we have

$$
\left(\triangleleft_{\sigma}\right)^{\mathrm{TOIPd}}=\triangleleft_{\mathrm{bt}(\sigma)}, \quad\left(\triangleleft_{\left[\sigma, \sigma^{\prime}\right]}\right)^{\mathrm{TOIPd}}=\triangleleft_{\left[\mathrm{bt}(\sigma), \mathrm{bt}\left(\sigma^{\prime}\right)\right]} \quad \text { and } \quad\left(\triangleleft_{\pi}\right)^{\mathrm{TOIPd}}=\triangleleft_{\mathrm{st}(\pi)} .
$$

Example 2.32. Compare Figures 6 and 11, Figures 7 and 12, and Figures 8 and 14 .

2.3. From Permutreehedra. Extending Sections 2.1 and 2.2, we describe further relevant families of posets corresponding to the elements, the faces, and the intervals in the permutreehedra introduced in [14]. This provides a wider range of examples and uniform proofs, at the cost of increasing the technicalities.

2.3.1. Permutree Element Posets. We first recall from [14] the definition of permutrees.

Definition 2.33 ([14]). A permutree is a directed tree $\mathrm{T}$ with vertex set $\mathrm{V}$ endowed with a bijective vertex labeling $p: \mathrm{V} \rightarrow[n]$ such that for each vertex $v \in \mathrm{V}$,

(1) $v$ has one or two parents (outgoing neighbors), and one or two children (incoming neighbors);

(2) if $v$ has two parents (resp. children), then all labels in the left ancestor (resp. descendant) subtree of $v$ are smaller than $p(v)$ while all labels in the right ancestor (resp. descendant) subtree of $v$ are larger than $p(v)$.

The orientation of a permutree $\mathrm{T}$ is $\mathbb{O}(\mathrm{T})=\left(n, \mathbb{O}^{+}, \mathbb{O}^{-}\right)$where $\mathbb{O}^{+}$is the set of labels of the nodes with two parents while $\mathbb{O}^{-}$is the set of labels of the nodes with two children. Note that there is a priori no conditions on these sets $\mathbb{O}^{+}$and $\mathbb{O}^{-}$: they can be empty, they can intersect, etc. For a given orientation $\mathbb{O}=\left(n, \mathbb{O}^{+}, \mathbb{O}^{-}\right)$, we denote by $\mathrm{PT}(\mathbb{O})$ the set of permutrees with orientation $\mathbb{O}$.

Figure 15 gives four examples of permutrees. While the first is generic, the other three show that specific permutrees encode relevant combinatorial objects, depending on their orientations:

\begin{tabular}{c||c|c|c} 
orientation $\left(n, \mathbb{O}^{+}, \mathbb{O}^{-}\right)$ & $(n, \varnothing, \varnothing)$ & $(n, \varnothing,[n])$ & $(n,[n],[n])$ \\
\hline combinatorial objects & permutations & binary trees & binary sequences
\end{tabular}

Another specific family of permutrees are the Cambrian trees of [2] obtained when $\mathbb{O}^{+} \sqcup \mathbb{O}^{-}=[n]$. See [14] for more details on the interpretation of all these 
combinatorial objects as permutrees. We use drawing conventions from [14]: nodes are ordered by their labels from left to right, edges are oriented from bottom to top, and we draw a red wall separating the two parents or the two children of a node. Condition (2) in Definition 2.33 says that no black edge can cross a red wall.

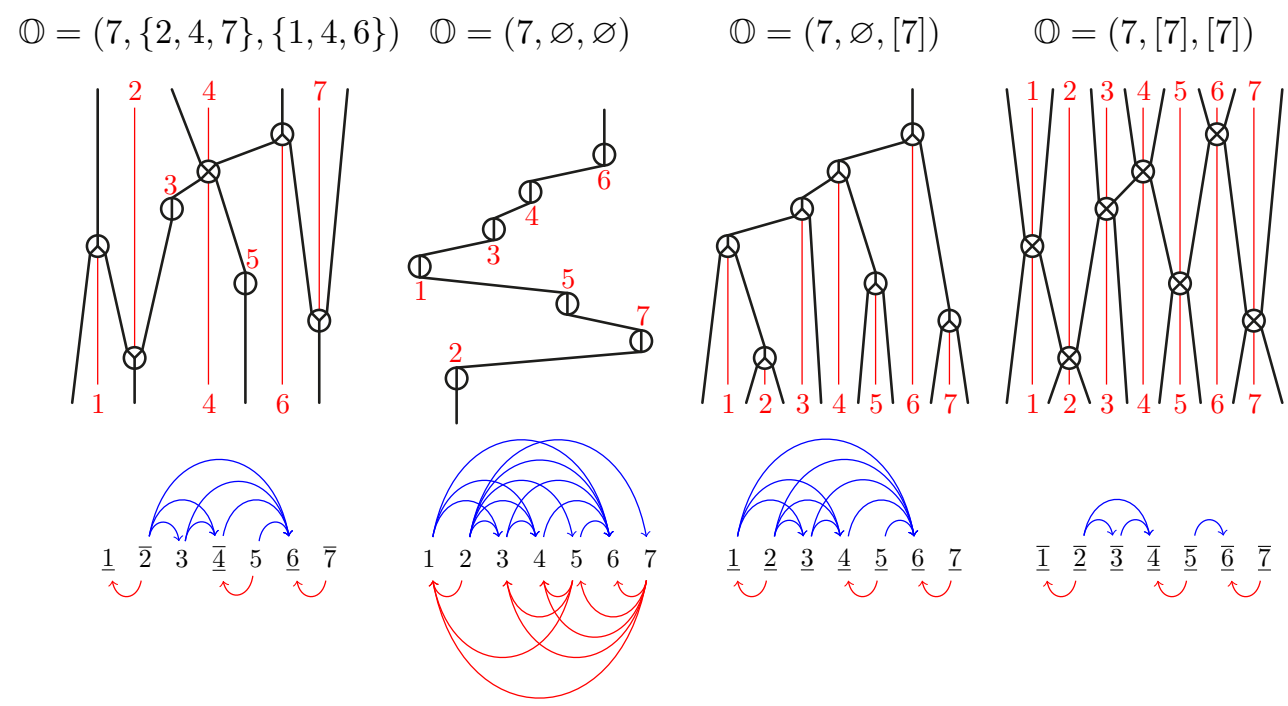

Figure 15. Four examples of permutrees $\mathrm{T}$ (top) with their posets $\triangleleft_{\mathrm{T}}$ (bottom). While the first is generic, the last three illustrate specific orientations corresponding to permutations, binary trees, and binary sequences.

For a permutree $\mathrm{T}$, we denote by $\triangleleft_{\mathrm{T}}$ the transitive closure of $\mathrm{T}$. That is to say, $i \triangleleft_{\mathrm{T}} j$ if and only if there is an oriented path from $i$ to $j$ in T. See Figure 15 for illustrations. To visualize the orientation $\mathbb{O}$ in the poset $\triangleleft_{\mathrm{T}}$, we overline (resp. underline) the elements of $\mathbb{O}^{+}$(resp. of $\left.\mathbb{O}^{-}\right)$.

We say that $\triangleleft_{\mathrm{T}}$ is a permutree element poset and we denote by

$$
\operatorname{PEP}(\mathbb{O}):=\left\{\triangleleft_{\mathrm{T}} \mid \mathrm{T} \in \mathrm{PT}(\mathbb{O})\right\}
$$

the set of all permutree element posets for a given orientation $\mathbb{O}$. These posets will be characterized in Proposition 2.44. For the moment, we need the following properties from [14].

Proposition 2.34 ([14]). Fix an orientation $\mathbb{O}=\left(n, \mathbb{O}^{+}, \mathbb{O}^{-}\right)$of $[n]$.

(1) For a permutree $\mathrm{T} \in \mathrm{PT}(\mathbb{O})$, the set of linear extensions $\mathcal{L}(\mathrm{T})$ of $\triangleleft_{\mathrm{T}}$ is an interval in the weak order on $\mathfrak{S}(n)$ whose minimal element avoids the pattern $c a-b$ with $a<b<c$ and $b \in \mathbb{O}^{-}$(denoted $31 \underline{2}$ ) and the pattern $b-c a$ with $a<b<c$ and $b \in \mathbb{O}^{+}$(denoted $\overline{2} 31$ ), and whose maximal element avoids the pattern $a c-b$ with $a<b<c$ and $b \in \mathbb{O}^{-}$(denoted 132) and the pattern $b-a c$ with $a<b<c$ and $b \in \mathbb{O}^{+}$(denoted $\overline{2} 31$ ).

(2) The collection of sets $\mathcal{L}(\mathrm{T}):=\left\{\right.$ linear extensions of $\left.\triangleleft_{\mathrm{T}}\right\}$ for all permutrees $\mathrm{T} \in \mathrm{PT}(\mathbb{O})$ forms a partition of $\mathfrak{S}(n)$. This defines a surjection $\Psi_{\mathbb{O}}$ from $\mathfrak{S}(n)$ to $\mathrm{PT}(\mathbb{O})$, which sends a permutation $\sigma \in \mathfrak{S}(n)$ to the unique permutree $\mathrm{T} \in \mathrm{PT}(\mathbb{O})$ such that $\sigma \in \mathcal{L}(\mathrm{T})$. This surjection can be described directly as an insertion algorithm (we skip this description and refer the interested reader to [14] as it is not needed for the purposes of this paper). 
(3) This partition defines a lattice congruence of the weak order (see [14, 15, 16] for details). Therefore, the set of permutrees $\mathrm{PT}(\mathbb{O})$ is endowed with a lattice structure $\preccurlyeq$, called permutree lattice, defined by

$\mathrm{T} \preccurlyeq \mathrm{T}^{\prime} \Longleftrightarrow \exists \sigma, \sigma^{\prime} \in \mathfrak{S}(n)$ such that $\Psi_{\mathbb{O}}(\sigma)=\mathrm{T}, \Psi_{\mathbb{O}}\left(\sigma^{\prime}\right)=\mathrm{T}^{\prime}$ and $\sigma \preccurlyeq \sigma^{\prime}$

whose meet $\wedge_{\mathbb{O}}$ and join $\vee_{\mathbb{O}}$ are given by

$$
\mathrm{T} \wedge_{\mathbb{O}} \mathrm{T}^{\prime}=\Psi_{\mathbb{O}}\left(\sigma \wedge_{\mathfrak{S}} \sigma^{\prime}\right) \quad \text { and } \quad \mathrm{T} \vee_{\mathbb{O}} \mathrm{T}^{\prime}=\Psi_{\mathbb{O}}\left(\sigma \vee_{\mathfrak{S}} \sigma^{\prime}\right)
$$

for any representatives $\sigma, \sigma^{\prime} \in \mathfrak{S}(n)$ such that $\Psi_{\mathbb{O}}(\sigma)=\mathrm{T}$ and $\Psi_{\mathbb{O}}\left(\sigma^{\prime}\right)=\mathrm{T}^{\prime}$. In particular, $\mathrm{T} \preccurlyeq \mathrm{T}^{\prime}$ if and only if $\sigma \preccurlyeq \sigma^{\prime}$ where $\sigma$ and $\sigma^{\prime}$ denote the minimal (resp. maximal) linear extensions of $\mathrm{T}$ and $\mathrm{T}^{\prime}$ respectively.

(4) This lattice structure can equivalently be described as the transitive closure of right rotations in permutrees as described in [14].

(5) The minimal (resp. maximal) permutree in the permutree lattice is a left (resp. right) $\mathbb{O}$-comb: it is a chain where each vertex in $\mathbb{O}^{+}$has an additional empty left (resp. right) parent, while each vertex in $\mathbb{O}^{-}$has an additional empty right (resp. left) child.

For example, we obtain well-known lattice structures for specific orientations:

\begin{tabular}{c||c|c|c} 
orientation $\left(n, \mathbb{O}^{+}, \mathbb{O}^{-}\right)$ & $(n, \varnothing, \varnothing)$ & $(n, \varnothing,[n])$ & $(n,[n],[n])$ \\
\hline permutree lattice & weak order & Tamari lattice & boolean lattice
\end{tabular}

When $\mathbb{O}^{+} \sqcup \mathbb{O}^{-}=[n]$, the permutree lattices on Cambrian trees coincide with the type $A$ Cambrian lattices of $[2,16]$.

Now we establish the relationship between the permutree lattice on $\mathrm{PT}(\mathbb{O})$ and the weak order on $\operatorname{PEP}(\mathbb{O})$.

Proposition 2.35. For any permutrees $\mathrm{T}, \mathrm{T}^{\prime} \in \mathrm{PT}(\mathbb{O})$, we have $\mathrm{T} \preccurlyeq \mathrm{T}^{\prime}$ in the permutree lattice if and only if $\triangleleft_{\mathrm{T}} \preccurlyeq \triangleleft_{\mathrm{T}^{\prime}}$ in the weak order on posets.

Proof. By Proposition 2.34(2), a permutree admits both a minimal and a maximal linear extensions. It follows that $\operatorname{PEP}(\mathbb{O}) \subseteq \operatorname{WOIP}(n)$ and the weak order on $\operatorname{PEP}(\mathbb{O})$ is therefore given by

$$
\triangleleft_{\mathrm{T}} \preccurlyeq \triangleleft_{\mathrm{T}^{\prime}} \Longleftrightarrow \triangleleft_{\mathrm{T}}^{\text {minle }} \preccurlyeq \triangleleft_{\mathrm{T}^{\prime}}^{\text {minle }} \text { and } \triangleleft_{\mathrm{T}}^{\text {maxle }} \preccurlyeq \triangleleft_{\mathrm{T}^{\prime}}^{\text {maxle }}
$$

according to Proposition 2.6. However, we have already mentioned in Proposition 2.34(3) that the two conditions on the right are both equivalent to $\mathrm{T} \preccurlyeq \mathrm{T}^{\prime}$ in the permutree lattice.

REMARK 2.36. In fact, $\mathrm{T} \preccurlyeq \mathrm{T}^{\prime} \Longleftrightarrow \triangleleft_{\mathrm{T}} \preccurlyeq \triangleleft_{\mathrm{T}^{\prime}} \Longleftrightarrow \triangleleft_{\mathrm{T}}^{\operatorname{lnc}} \supseteq \triangleleft_{\mathrm{T}^{\prime}}^{\operatorname{lnc}} \Longleftrightarrow \triangleleft_{\mathrm{T}}^{\text {Dec }} \subseteq \triangleleft_{\mathrm{T}^{\prime}}^{\text {Dec }}$.

REMARK 2.37. Proposition 2.35 affirms that the subposet of the weak order on $\mathcal{P}$ induced by the set PEP $(\mathbb{O})$ is isomorphic to the permutree lattice on $\mathrm{PT}(\mathbb{O})$, and is thus a lattice. We will see in Remark 2.87 that the set PEP $(\mathbb{O})$ does not always induce a sublattice of the weak order on $\mathcal{P}(n)$. Theorem 2.85 will provide a sufficient condition on the orientation $\mathbb{O}$ for this property. In contrast, we will see in Theorem 2.88 that $\operatorname{PEP}(\mathbb{O})$ always induces a sublattice of the weak order on $\operatorname{PIP}(\mathbb{O})$ and thus also on $\operatorname{WOIP}(n)$.

2.3.2. Permutree Interval Posets. For two permutrees $\mathrm{T}, \mathrm{T}^{\prime} \in \mathrm{PT}(\mathbb{O})$ with $\mathrm{T} \preccurlyeq \mathrm{T}^{\prime}$, we denote by $\left[\mathrm{T}, \mathrm{T}^{\prime}\right]:=\left\{\mathrm{S} \in \mathrm{PT}(\mathbb{O}) \mid \mathrm{T} \preccurlyeq \mathrm{S} \preccurlyeq \mathrm{T}^{\prime}\right\}$ the permutree lattice interval between $\mathrm{T}$ and $\mathrm{T}^{\prime}$. As in Proposition 2.4, we can see this interval as the poset

$$
\triangleleft_{\left[\mathrm{T}, \mathrm{T}^{\prime}\right]}:=\bigcap_{\mathrm{T} \preccurlyeq \mathrm{S} \preccurlyeq \mathrm{T}^{\prime}} \triangleleft_{\mathrm{T}}=\triangleleft_{\mathrm{T}} \cap \triangleleft_{\mathrm{T}^{\prime}}=\triangleleft_{\mathrm{T}^{\prime}}^{\mathrm{Inc}} \cap \triangleleft_{\mathrm{T}}^{\mathrm{Dec}} .
$$


We say that $\triangleleft_{\left[\mathrm{T}, \mathrm{T}^{\prime}\right]}$ is a permutree interval poset, and we denote by

$$
\operatorname{PIP}(\mathbb{O}):=\left\{\triangleleft_{\left[\mathrm{T}, \mathrm{T}^{\prime}\right]} \mid \mathrm{T}, \mathrm{T}^{\prime} \in \mathrm{PT}(\mathbb{O}), \mathrm{T} \preccurlyeq \mathrm{T}^{\prime}\right\}
$$

the set of all permutree interval posets for a given orientation $\mathbb{O}$.

We first aim at a concrete characterization of the posets of $\operatorname{PIP}(\mathbb{O})$. Note that a poset is in $\mathrm{PIP}(\mathbb{O})$ if and only if it admits a weak order minimal linear extension avoiding the patterns $31 \underline{2}$ and $\overline{2} 31$, and a weak order maximal linear extension avoiding the patterns $13 \underline{2}$ and $\overline{2} 13$. Similar to our study of $\operatorname{IWOIP}(n)$ and $\operatorname{DWOIP}(n)$ in Section 2.1.4, it is practical to consider these conditions separately. We thus define the set IPIP $(\mathbb{O})$ (resp. DPIP $(\mathbb{O})$ ) of posets which admit a maximal (resp. minimal) linear extension that avoids the patterns $\overline{2} 13$ and $13 \underline{2}$ (resp. $\overline{2} 31$ and $31 \underline{2}$ ). In order to characterize these posets, we define

$$
\begin{aligned}
& \operatorname{IPIP}^{+}(\mathbb{O}):=\left\{\triangleleft \in \mathcal{P}(n) \mid \forall a<b<c \text { with } b \in \mathbb{O}^{+}, a \triangleleft c \Longrightarrow a \triangleleft b\right\}, \\
& \operatorname{IPIP}^{-}(\mathbb{O}):=\left\{\triangleleft \in \mathcal{P}(n) \mid \forall a<b<c \text { with } b \in \mathbb{O}^{-}, a \triangleleft c \Longrightarrow b \triangleleft c\right\}, \\
& \operatorname{IPIP}^{ \pm}(\mathbb{O}):=\operatorname{IPIP}^{+}(\mathbb{O}) \cap \operatorname{IPIP}^{-}(\mathbb{O}),
\end{aligned}
$$

and similarly

$$
\begin{aligned}
& \operatorname{DPIP}^{+}(\mathbb{O}):=\left\{\triangleleft \in \mathcal{P}(n) \mid \forall a<b<c \text { with } b \in \mathbb{O}^{+}, a \triangleright c \Longrightarrow b \triangleright c\right\}, \\
& \operatorname{DPIP}^{-}(\mathbb{O}):=\left\{\triangleleft \in \mathcal{P}(n) \mid \forall a<b<c \text { with } b \in \mathbb{O}^{-}, a \triangleright c \Longrightarrow a \triangleright b\right\}, \\
& \operatorname{DPIP}^{ \pm}(\mathbb{O}):=\operatorname{IPIP}^{+}(\mathbb{O}) \cap \operatorname{IPIP}^{-}(\mathbb{O}) .
\end{aligned}
$$

Proposition 2.38. For any orientation $\mathbb{O}$ of $[n]$, we have

$$
\operatorname{IPIP}(\mathbb{O})=\operatorname{IWOIP}(n) \cap \operatorname{IPIP}^{ \pm}(\mathbb{O}) \quad \text { and } \quad \operatorname{DPIP}(\mathbb{O})=\operatorname{DWOIP}(n) \cap \operatorname{DPIP}^{ \pm}(\mathbb{O}) .
$$

Proof. Consider $\triangleleft \in$ IWOIP and let $\triangleleft^{\text {maxle }}=\triangleleft \cup\{(b, a) \mid a<b$ incomparable in $\triangleleft\}$ be its maximal linear extension (see the proof of Proposition 2.12). Assume first that there is $a<b<c$ with $b \in \mathbb{O}^{+}$such that $a \triangleleft c$ while $a \nless b$. Then we obtain $b \triangleleft^{\text {maxle }} a \triangleleft^{\text {maxle }} c$ which is a $\overline{2} 13$-pattern in $\triangleleft^{\text {maxle }}$. Reciprocally, if $\triangleleft^{\text {maxle }}$ contains a $\overline{2} 13$-pattern $b \triangleleft^{\text {maxle }} a \triangleleft^{\text {maxle }} c$ with $a<b<c$ and $b \in \mathbb{O}^{+}$, then $a \triangleleft c$ while $a \nless b$ by definition of $\triangleleft^{\text {maxle }}$. We conclude that $\triangleleft^{\text {maxle }}$ avoids the pattern $\overline{2} 13$ if and only if $\triangleleft \in \operatorname{IPIP}^{+}(\mathbb{O})$. The proof for the other patterns is similar.

Corollary 2.39. A poset $\triangleleft$ is in $\mathrm{PIP}(\mathbb{O})$ if and only if it is in $\operatorname{WOIP}(n)$ (see characterization in Proposition 2.5) and satisfies the conditions of $\operatorname{IPIP}^{+}(\mathbb{O}), \operatorname{IPIP}^{-}(\mathbb{O})$, $\mathrm{DPIP}^{+}(\mathbb{O})$ and $\mathrm{DPIP}^{-}(\mathbb{O})$.

REMARK 2.40. Similarly to Remark 2.14, note that it suffices to check these conditions only for all cover relations $a \triangleleft c$ and $a \triangleright c$ in $\triangleleft$.

Some illustrations are given in Figure 16. The leftmost poset is not in $\operatorname{PIP}(\mathbb{O})$ : $\{1,2,3\}$ does not satisfy IPIP ${ }^{-}(\mathbb{O}),\{2,3,5\}$ does not satisfy $\operatorname{IPIP}^{+}(\mathbb{O}),\{3,4,6\}$ does not satisfy DWOIP $(6)$, and $\{3,5,6\}$ does not satisfy $\operatorname{DPIP}^{-}(\mathbb{O})$. The other two posets of Figure 16 are both in PIP(O).

Now we describe the weak order on $\operatorname{PIP}(\mathbb{O})$.

Proposition 2.41. For any $\mathrm{S} \preccurlyeq \mathrm{S}^{\prime}$ and $\mathrm{T} \preccurlyeq \mathrm{T}^{\prime}$, we have $\triangleleft_{\left[\mathrm{S}, \mathrm{S}^{\prime}\right]} \preccurlyeq \triangleleft_{\left[\mathrm{T}, \mathrm{T}^{\prime}\right]}$ if and only if $\mathrm{S} \preccurlyeq \mathrm{T}$ and $\mathrm{S}^{\prime} \preccurlyeq \mathrm{T}^{\prime}$.

Proof. The proof is similar to that of Proposition 2.6.

We immediately derive that the weak order on PIP $(\mathbb{O})$ has the lattice structure of a product. 


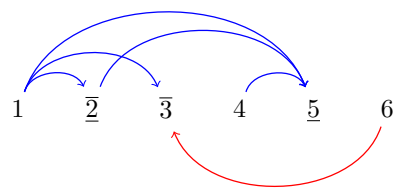

$\notin \operatorname{PIP}(\mathbb{O})$

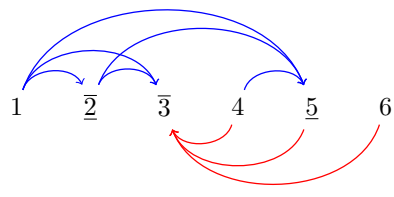

$\in \operatorname{PIP}(\mathbb{O}) \backslash \operatorname{PEP}(\mathbb{O})$

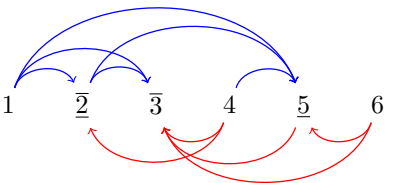

$\in \operatorname{PEP}(\mathbb{O})$

FiguRE 16. Examples and counterexamples of elements in $\operatorname{PIP}(\mathbb{O})$ and $\operatorname{PEP}(\mathbb{O})$, where $\mathbb{O}=(6,\{2,3\},\{2,5\})$.

Corollary 2.42. The weak order on $\mathrm{PIP}(\mathbb{O})$ is a lattice whose meet and join are given by

$$
\begin{aligned}
\triangleleft_{\left[\mathrm{S}, \mathrm{S}^{\prime}\right]} & \wedge_{\mathrm{PIP}(\mathbb{O})} \triangleleft_{\left[\mathrm{T}, \mathrm{T}^{\prime}\right]}=\triangleleft_{\left[\mathrm{S} \wedge_{\mathbb{O}} \mathrm{T}, \mathrm{S}^{\prime} \wedge_{\mathbb{O}} \mathrm{T}^{\prime}\right]} \\
\text { and } \triangleleft_{\left[\mathrm{S}, \mathrm{S}^{\prime}\right]} \vee_{\mathrm{PIP}(\mathbb{O})} \triangleleft_{\left[\mathrm{T}, \mathrm{T}^{\prime}\right]} & =\triangleleft_{\left[\mathrm{S} \vee_{\mathbb{O}} \mathrm{T}, \mathrm{S}^{\prime} \vee_{\mathbb{O}} \mathrm{T}^{\prime}\right]}
\end{aligned}
$$

REMARK 2.43. As illustrated by $\operatorname{WOIP}(n)$, the set PIP $(\mathbb{O})$ does not always induce a sublattice of the weak order on $\mathcal{P}(n)$. Theorem 2.77 will provide a sufficient condition on the orientation $\mathbb{O}$ for this property. In contrast, we will see in Theorem 2.82 that $\operatorname{PIP}(\mathbb{O})$ always induces a sublattice of the weak order on $\operatorname{WOIP}(n)$.

2.3.3. Characterization of $\operatorname{PEP}(\mathbb{O})$. We are now ready to give a characterization of the posets of PEP $(\mathbb{O})$ left open in Section 2.3.1. We need one additional definition. For an orientation $\mathbb{O}$ of $[n]$, an $\mathbb{O}$-snake in a poset $\triangleleft$ is a sequence $x_{0}<x_{1}<\cdots<x_{k}<x_{k+1}$ such that

- either $x_{0} \triangleleft x_{1} \triangleright x_{2} \triangleleft x_{3} \triangleright \cdots$ with $\left\{x_{i} \mid i \in[k]\right.$ odd $\} \subseteq \mathbb{O}^{-}$and $\left\{x_{i} \mid i \in[k]\right.$ even $\} \subseteq \mathbb{O}^{+}$,

- or $x_{0} \triangleright x_{1} \triangleleft x_{2} \triangleright x_{3} \triangleleft \cdots$ with $\left\{x_{i} \mid i \in[k]\right.$ odd $\} \subseteq \mathbb{O}^{+}$and $\left\{x_{i} \mid i \in[k]\right.$ even $\} \subseteq \mathbb{O}^{-}$,

as illustrated in Figure 17.
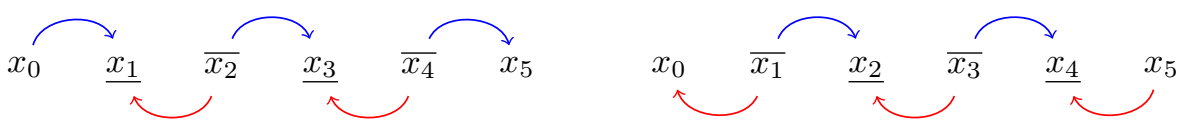

Figure 17. Two $\mathbb{O}$-snakes joining $x_{0}$ to $x_{5}$. The set $\mathbb{O}^{+}\left(\right.$resp. $\left.\mathbb{O}^{-}\right)$ must contain at least the overlined (resp. underlined) integers.

We say that the $\mathbb{O}$-snake $x_{0}<x_{1}<\cdots<x_{k}<x_{k+1}$ joins $x_{0}$ to $x_{k+1}$ and has length $k$. Note that, by definition, we consider the relations $x \triangleleft y$ or $x \triangleright y$ themselves as (degenerate, length 0 ) $\mathbb{O}$-snakes between $x$ and $y$.

Proposition 2.44. A poset $\triangleleft \in \mathcal{P}(n)$ is in $\mathrm{PEP}(\mathbb{O})$ if and only if it is in $\mathrm{PIP}(\mathbb{O})$ (see characterization in Corollary 2.39) and it admits an $\mathbb{O}$-snake between any two values of $[n]$.

Figure 16 illustrates this proposition: the middle poset is not in $\operatorname{PEP}(\mathbb{O})$, since there is no $\mathbb{O}$-snake between 1 and 4 nor between 1 and 6 . In contrast, the rightmost poset is in $\operatorname{PEP}(\mathbb{O})$, as $1 \triangleleft \underline{2} \triangleright 4$ is an $\mathbb{O}$-snake between 1 and 4 and $1 \triangleleft \underline{5} \triangleright 6$ is an (1)-snake between 1 and 6 . 
Proof or Proposition 2.44. Assume that $\triangleleft \in \mathrm{PEP}(\mathbb{O})$, and let $\mathrm{T} \in \mathrm{PT}(\mathbb{O})$ be the permutree such that $\triangleleft=\triangleleft_{\mathrm{T}}$. Then $\triangleleft$ is certainly in $\operatorname{PIP}(\mathbb{O})$. Now any two values $x, y \in[n]$ are connected by a (non-oriented) path in $\mathrm{T}$, and recalling the local optima along this path provides an $\mathbb{O}$-snake joining $x$ and $y$.

Reciprocally, consider $\triangleleft \in \operatorname{PIP}(\mathbb{O})$ such that there is an $\mathbb{O}$-snake between any two values of $[n]$. We need the following two intermediate claims, proved in detail in Appendix A.2.

Claim 2.45. For any $u, v, w \in[n]$ with $u<w$,

- if $u \triangleleft v$ and $v \triangleright w$ are cover relations of $\triangleleft$, then $u<v<w$ and $v \in \mathbb{O}^{-}$;

- if $u \triangleright v$ and $v \triangleleft w$ are cover relations of $\triangleleft$, then $u<v<w$ and $v \in \mathbb{O}^{+}$.

Claim 2.46. Let $x_{0}, \ldots, x_{p} \in[n]$ be a path in the Hasse diagram of $\triangleleft$ (i.e. $x_{i-1} \triangleleft x_{i}$ or $x_{i-1} \triangleright x_{i}$ are cover relations in $\triangleleft$ for any $\left.i \in[p]\right)$. Assume moreover that $x_{0} \in \mathbb{O}^{-}$ and $x_{0} \triangleright x_{1}$, or that $x_{0} \in \mathbb{O}^{+}$and $x_{0} \triangleleft x_{1}$. Then all $x_{i}$ are on the same side of $x_{0}$, i.e. $x_{0}<x_{1} \Longleftrightarrow x_{0}<x_{i}$ for all $i \in[p]$.

Claims 2.45 and 2.46 show that the Hasse diagram of $\triangleleft$ is a permutree:

- it is connected since any two values are connected by a snake,

- it cannot contain a cycle (otherwise, since this cycle cannot be oriented, there exist three distinct vertices $u, v, w$ in this cycle with $u \triangleleft v \triangleright w$. Claim 2.45 ensures that $u<v<w$ and $v \in \mathbb{O}^{-}$. Since there is a path $v=x_{0}, w=x_{1}, x_{2}, \ldots, x_{p}=u$ in the Hasse diagram of $\triangleleft$ with $v \in \mathbb{O}^{-}$ and $v \triangleright w$, Claim 2.46 affirms that $u$ and $w$ are on the same side of $v$, a contradiction), and

- it fulfills the local conditions of Definition 2.33 to be a permutree (Claim 2.45 shows Condition (1) of Definition 2.33, and Claim 2.46 shows Condition (2) of Definition 2.33).

For further purposes, we will need the following lemma to check the existence of (O)-snakes.

LEMmA 2.47. Let $\triangleleft \in \mathcal{P}(n)$ and $a<c$ be incomparable in $\triangleleft$. The following are equivalent:

(1) There is an $\mathbb{O}$-snake between $a$ and $c$,

(2) $\exists a<b<c$ such that there is an $\mathbb{O}$-snake between $a$ and $b$, and either $b \in \mathbb{O}^{-}$ and $b \triangleright c$, or $b \in \mathbb{O}^{+}$and $b \triangleleft c$,

(3) $\exists a<b<c$ such that there is an $\mathbb{O}$-snake between $b$ and $c$, and either $b \in \mathbb{O}^{-}$ and $a \triangleleft b$, or $b \in \mathbb{O}^{+}$and $a \triangleright b$.

Proof. The implication $(1) \Rightarrow(2)$ is immediate, considering $b=x_{k}$. Assume now that $\triangleleft$ and $\{a, c\}$ satisfy (2). Let $b$ be given by (2) and let $a<x_{1}<\cdots<x_{k}<b$ be an $\mathbb{O}$-snake between $a$ and $b$. If $x_{k} \triangleleft b \triangleright c$ (or similarly if $x_{k} \triangleright b \triangleleft c$ ), then $a<x_{1}<\cdots<b<c$ is a $\mathbb{O}$-snake between $a$ and $c$. In contrast, if $x_{k} \triangleleft b \triangleleft c$ (or similarly if $x_{k} \triangleright b \triangleright c$ ), then $x_{k} \triangleleft c$ (resp. $x_{k} \triangleright c$ ) by transitivity of $\triangleleft$, so that $a<x_{1}<\cdots<x_{k}<c$ is an $\mathbb{O}$-snake between $a$ and $c$. Therefore, (1) $\Longleftrightarrow(2)$. The proof of $(1) \Longleftrightarrow(3)$ is identical.

2.3.4. Permutree Face Posets. The permutrees of $\mathrm{PT}(\mathbb{O})$ correspond to the vertices of the $\mathbb{O}$-permutreehedron $\mathrm{PT}(\mathbb{O})$ constructed in $[14]$. The precise definition of these polytopes is not needed here. Following Figure 15, we illustrate in Figure 18 classical polytopes that arise as permutreehedra for specific orientations:

\begin{tabular}{c||c|c|c} 
orientation $\left(n, \mathbb{O}^{+}, \mathbb{O}^{-}\right)$ & $(n, \varnothing, \varnothing)$ & $(n, \varnothing,[n])$ & $(n,[n],[n])$ \\
\hline permutreehedron & permutahedron & Loday's associahedron & parallelepiped
\end{tabular}


When $\mathbb{O}^{+} \sqcup \mathbb{O}^{-}=[n]$, the permutreehedra coincide with the associahedra of C. Hohlweg and C. Lange $[2,5,8]$.

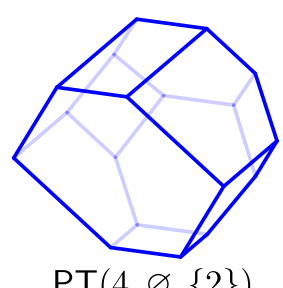

$\mathrm{PT}(4, \varnothing,\{2\})$

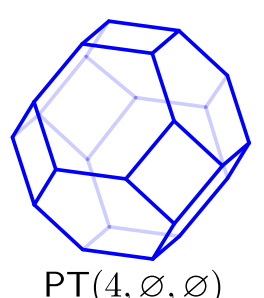

$\mathrm{PT}(4, \varnothing, \varnothing)$

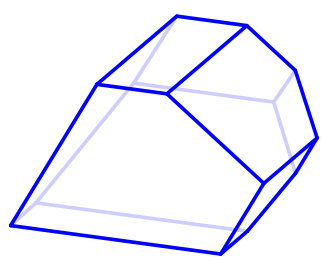

$\operatorname{PT}(4, \varnothing,[4])$

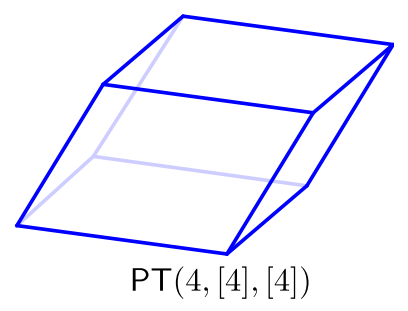

$\mathrm{PT}(4,[4],[4])$

Figure 18. Four examples of permutreehedra. While the first is generic, the last three are a permutahedron, an associahedron [9], and a parallelepiped.

We now consider all the faces of the $\mathbb{O}$-permutreehedron. As shown in [14], they correspond to Schröder $\mathbb{O}$-permutrees, defined as follows.

Definition 2.48 ([14]). For an orientation $\mathbb{O}$ on $[n]$ and a subset $X \subseteq[n]$, we let $X^{-}:=X \cap \mathbb{O}^{-}$and $X^{+}:=X \cap \mathbb{O}^{+}$. A Schröder $\mathbb{O}$-permutree is a directed tree $\mathrm{S}$ with vertex set $\mathrm{V}$ endowed with a vertex labeling $p: \mathrm{V} \rightarrow 2^{[n]} \backslash \varnothing$ such that

(1) the labels of $\mathrm{S}$ partition $[n]$, i.e. $v \neq w \in \mathrm{V} \Longrightarrow p(v) \cap p(w)=\varnothing$ and $\bigcup_{v \in \mathrm{V}} p(v)=[n]$

(2) each vertex $v \in \mathrm{V}$ has one incoming (resp. outgoing) subtree $\mathrm{S}_{v}^{I}$ (resp. $\mathrm{S}_{I}^{v}$ ) for each interval I of $[n] \backslash p(v)^{-}\left(\right.$resp. of $\left.[n] \backslash p(v)^{+}\right)$and all labels of $\mathrm{S}_{v}^{I}$ (resp. of $\mathrm{S}_{I}^{v}$ ) are subsets of $I$.

We denote by $\operatorname{SchrPT}(\mathbb{O})$ the set of Schröder $\mathbb{O}$-permutrees.

For example, in the leftmost Schröder permutree of Figure 19, the vertices are labeled by the sets $\{1,2\},\{3\},\{4,6\},\{5\}$, and $\{6,7\}$. The vertex $v$ labeled by $p(v)=\{4,6\}$ has 3 incoming subtrees included in the 3 intervals of $[n] \backslash p(v)^{-}=[n] \backslash\{4,6\}=\{1,2,3\} \sqcup\{5\} \sqcup\{7\}$ and 2 (empty) outgoing subtrees included in the 2 intervals of $[n] \backslash p(v)^{+}=[n] \backslash\{4\}=\{1,2,3\} \sqcup\{5,6,7\}$.

Following Figure 15, we have represented in Figure 19 four Schröder permutrees, where the last three encode relevant combinatoiral objects obtained for specific orientations:

\begin{tabular}{c||c|c|c} 
orientation $\left(n, \mathbb{O}^{+}, \mathbb{O}^{-}\right)$ & $(n, \varnothing, \varnothing)$ & $(n, \varnothing,[n])$ & $(n,[n],[n])$ \\
\hline combinatorial objects & ordered partitions & Schröder trees & ternary sequences
\end{tabular}

Another specific family of Schröder permutrees are the Schröder Cambrian trees of [2] obtained when $\mathbb{O}^{+} \sqcup \mathbb{O}^{-}=[n]$. We refer again to [14] for more details on the interpretation of all these combinatorial objects as permutrees, and we still use the drawing conventions of [14].

An $\mathbb{O}$-permutree $\mathrm{T}$ belongs to a face of the permutreehedron PT $(\mathbb{O})$ corresponding to a Schröder $\mathbb{O}$-permutree $\mathrm{S}$ if and only if $\mathrm{S}$ is obtained by edge contractions in $\mathrm{T}$. The set of such $\mathbb{O}$-permutrees is the interval $\left[\mathrm{T}^{\min }(\mathrm{S}), \mathrm{T}^{\max }(\mathrm{S})\right]$ of the $\mathbb{O}$-permutree lattice, where the minimal (resp. maximal) tree $\mathrm{T}^{\min }(\mathrm{S})\left(\right.$ resp. $\left.\mathrm{T}^{\max }(\mathrm{S})\right)$ is obtained by replacing the nodes of S by left (resp. right) combs as illustrated in Figure 20. To be more precise, we need additional notations. For an interval $I=[i, j]$ of integers, define $\bar{I}:=[i-1, j+1]$. For each edge $v \rightarrow w$ in S, we let $I_{v}^{w}$ (resp. $J_{v}^{w}$ ) denote the interval of $[n] \backslash p(v)^{+}$(resp. of $\left.[n] \backslash p(w)^{-}\right)$such that $p(w) \subseteq I_{v}^{w}$ (resp. $\left.p(v) \subseteq J_{v}^{w}\right)$. 


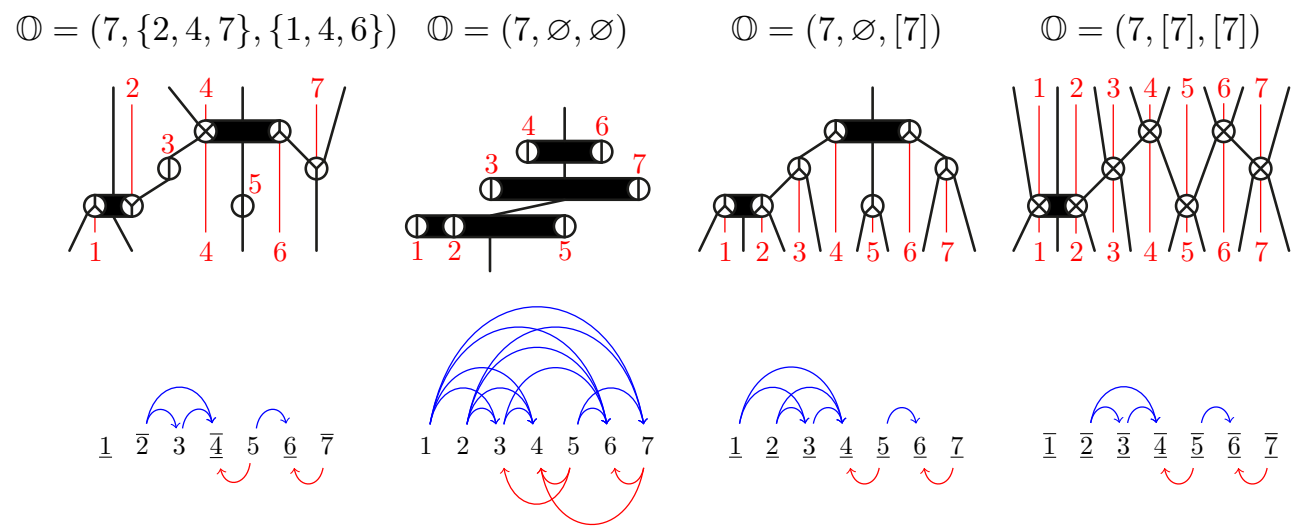

FiguRE 19. Four examples of Schröder permutrees S (top) with their posets $\triangleleft_{\mathrm{S}}$ (bottom). While the first is generic, the last three illustrate specific orientations corresponding to ordered partitions, Schröder trees, Schröder Cambrian trees, and ternary sequences.

The minimal and maximal permutrees in the face corresponding to the Schröder permutree $\mathrm{S}$ are then described as follows:

(1) $\mathrm{T}^{\mathrm{min}}(\mathrm{S})$ is the $\mathbb{O}$-permutree obtained from the left combs on the subsets $p(v)$ for $v$ vertex of $\mathrm{S}$ by adding the edges connecting $\max \left(p(v) \cap \bar{I}_{v}^{w}\right)$ with $\min \left(p(w) \cap \bar{J}_{v}^{w}\right)$ for all edges $v \rightarrow w$ in $\mathrm{S}$.

(2) $\mathrm{T}^{\max }(\mathrm{S})$ is the $\mathbb{O}$-permutree obtained from the right combs on the subsets $p(v)$ for $v$ vertex of $\mathrm{S}$ by adding the edges connecting $\min \left(p(v) \cap \bar{I}_{v}^{w}\right)$ with $\max \left(p(w) \cap \bar{J}_{v}^{w}\right)$ for all edges $v \rightarrow w$ in $\mathrm{S}$.

For example, consider the edge $v=\{5\} \rightarrow\{4,6\}=w$ in the Schröder permutree of Figure 20. We have $I_{v}^{w}=[n]$ and $J_{v}^{w}=\{5\}$. In $\mathrm{T}^{\min }(\mathrm{S})$, we create the left comb $4 \rightarrow 6$ and we add the edge $5=\max (\{5\} \cap[n]) \rightarrow \min (\{4,6\} \cap\{4,5,6\})=4$. Similarly, in $\mathrm{T}^{\max }(\mathrm{S})$, we create the right comb $6 \rightarrow 4$ and we add the edge $5=\min (\{5\} \cap[n]) \rightarrow \max (\{4,6\} \cap\{4,5,6\})=6$.

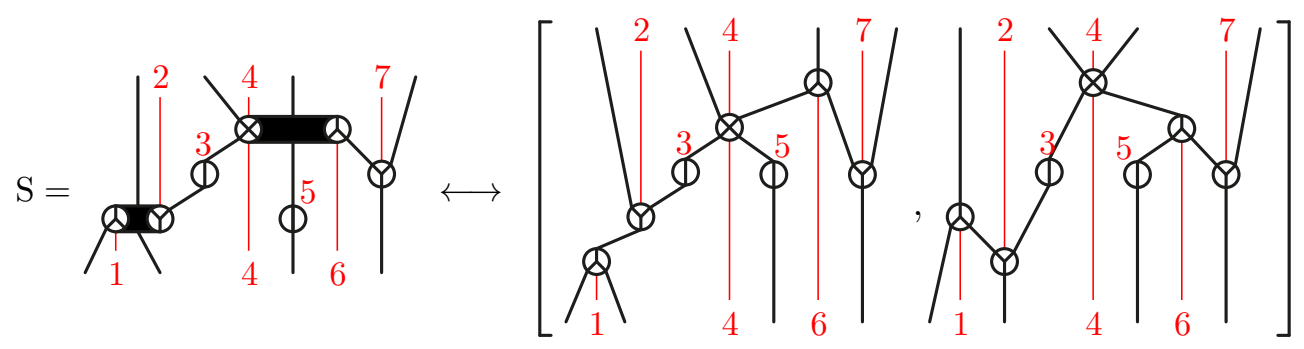

FiguRE 20. A Schröder permutree S (left) can be seen as an interval $\left[\mathrm{T}^{\min }(\mathrm{S}), \mathrm{T}^{\max }(\mathrm{S})\right]$ in the permutree lattice (right).

For a Schröder permutree $S$, we define $\triangleleft_{S}=\triangleleft_{\left[T^{\min }(S), T^{\max }(S)\right]}$. Examples are given in Figure 19. One easily checks that $\triangleleft_{\mathrm{S}}$ could also be defined as the transitive closure of all relations $i \triangleleft_{\mathrm{S}} j$ for all $i \in p(v) \cap \bar{I}_{v}^{w}$ and $j \in p(w) \cap \bar{J}_{v}^{w}$ for all edges $v \rightarrow w$ in S. For edge $\{5\} \rightarrow\{4,6\}$ in the leftmost Schröder permutree of Figure 19, this corresponds to the relations $4 \triangleright 5 \triangleleft 6$ of the poset. Note that 
- an $\mathbb{O}$-permutree $\mathrm{T}$ belongs to the face of the permutreehedron PT(O) corresponding to a Schröder $\mathbb{O}$-permutree $\mathrm{S}$ if and only if $\triangleleft_{\mathrm{T}}$ is an extension of $\triangleleft_{\mathrm{S}}$,

- the linear extensions of $\triangleleft_{\mathrm{S}}$ are precisely the linear extensions of $\triangleleft_{\mathrm{T}}$ for all (O)-permutrees $\mathrm{T}$ which belong to the face of the permutreehedron $\mathrm{PT}(\mathbb{O})$ corresponding to $\mathrm{S}$.

We say that $\triangleleft_{\mathrm{S}}$ is a permutree face poset, and we denote by

$$
\operatorname{PFP}(\mathbb{O}):=\left\{\triangleleft_{\mathrm{S}} \mid \mathrm{S} \in \operatorname{SchrPT}(\mathbb{O})\right\}
$$

the set of all permutree face posets. We now characterize these posets.

Proposition 2.49. A poset $\triangleleft \in \mathcal{P}(n)$ is in $\operatorname{PFP}(\mathbb{O})$ if and only if it is in $\operatorname{PIP}(\mathbb{O})$ and for any $a<c$ incomparable in $\triangleleft$,

(\$) $\exists a<b<c$ such that $b \in \mathbb{O}^{+}$and $a \not b \not c$ or $b \in \mathbb{O}^{-}$and $a \not b \not c$,

(\$) or $\forall a<b<c$ we have $a \triangleleft b \Longleftrightarrow b \triangleright c$ and $a \triangleright b \Longleftrightarrow b \triangleleft c$.

This property is illustrated on the leftmost poset of Figure 19. For example, 1 and 2 are neighbors and thus satisfy (\$), 1 and 3 satisfy (\$) with $b=2,4$ and 6 satisfy (\$), etc.

Proof of Proposition 2.49. Assume that $\triangleleft \in \operatorname{PFP}(\mathbb{O})$, and consider the Schröder permutree $\mathrm{S}$ such that $\triangleleft=\triangleleft_{\mathrm{S}}$. Then $\triangleleft=\triangleleft_{\mathrm{S}}=\triangleleft_{\left[\mathrm{T}^{\min }(\mathrm{S}), \mathrm{T}^{\max }(\mathrm{S})\right]}$ belongs to $\operatorname{PIP}(\mathbb{O})$. Moreover, any $a<c$ incomparable in $\triangleleft_{\mathrm{S}}$

(1) satisfy (\$) when either $a, c$ belong to distinct vertices of $\mathrm{S}$ separated by a wall, or $a, c$ belong to the same vertex $v$ of $\mathrm{S}$ but there is another $b$ in $v$ with $b \in \mathbb{O}^{+} \cup \mathbb{O}^{-}$and $a<b<c$,

(2) satisfy (\$) when $a, c$ belong to the same vertex $v$ of $\mathrm{S}$ and $b \notin \mathbb{O}^{+} \cup \mathbb{O}^{-}$for any $a<b<c$ in $v$.

This shows one implication of the statement. Before proving the reciprocal implication, let us comment a little more to give some useful intuition. Note that two consecutive elements $a<c$ in a vertex $v$ of $\mathrm{S}$ satisfy (\$) and not ( $)$. In particular, if all $a<c$ incomparable in $\triangleleft_{S}$ satisfy (A), then $S$ is just a permutree. In general, the posets $\triangleleft_{\mathrm{T}^{\min }(\mathrm{S})}$ and $\triangleleft_{\mathrm{T}^{\min }(\mathrm{S})}$ corresponding to the minimal and maximal (O-permutrees in the face corresponding to $\mathrm{S}$ are given by

$$
\triangleleft_{\mathrm{T}^{\min }(\mathrm{S})}=\left(\triangleleft \cup\left\{(a, c) \mid a<c \text { incomparable in } \triangleleft_{\mathrm{S}} \text { and not satisfying }(\boldsymbol{A})\right\}\right)^{\text {tc }}
$$

and $\triangleleft_{\mathrm{T}^{\max }(\mathrm{S})}=\left(\triangleleft \cup\left\{(c, a) \mid a<c \text { incomparable in } \triangleleft_{\mathrm{S}} \text { and not satisfying }(\boldsymbol{\rho})\right\}\right)^{\mathrm{tc}}$.

Consider now an arbitrary poset $\triangleleft \in \mathrm{PIP}(\mathbb{O})$ such that any $a<c$ incomparable in $\triangleleft$ satisfy $(\mathbf{A})$ or $(\mathbf{s})$. The previous observation motivates the following claim (see Appendix A.3 for the proof).

ClaIm 2.50. If any $a<c$ incomparable in $\triangleleft$ satisfy $(\boldsymbol{A})$, then $\triangleleft \in \operatorname{PEP}(\mathbb{O}) \subset \operatorname{PFP}(\mathbb{O})$.

Suppose now that some $a<c$ incomparable in $\triangleleft$ do not satisfy $(\boldsymbol{A})$. The idea of our proof is to return to the previous claim by considering the auxiliary poset

$$
\varangle:=(\triangleleft \cup\{(a, c) \mid a<c \text { incomparable in } \triangleleft \text { and not satisfying }(\mathbf{A})\})^{\text {tc }} \text {. }
$$

Claim 2.51. We have $\triangleleft^{\text {Inc }} \subseteq \triangleleft^{\text {Inc }}$ and $\triangleleft^{\text {Dec }}=\triangleleft^{\text {Dec }}$.

ClaIm 2.52. If $\triangleleft \in \mathrm{PIP}(\mathbb{O})$ and any $a<c$ incomparable in $\triangleleft$ satisfy $(\mathbf{A})$ or $(\boldsymbol{\beta})$, then $\bullet \in \operatorname{PIP}(\mathbb{O})$ and any $a<c$ incomparable in $\varangle$ satisfy $(\mathbf{M})$. 
Combining Claims 2.50 and 2.52, we obtain that there exists a permutree $\mathrm{T}$ such that $\boldsymbol{\Psi}=\triangleleft_{\mathrm{T}}$. Intuitively, $\mathrm{T}$ is the minimal permutree in the face that will correspond to $\triangleleft$. To find the Schröder permutree of this face, we thus just need to contract some edges in T. We therefore consider the Schröder permutree $\mathrm{S}$ obtained from $\mathrm{T}$ by contracting all edges that appear in the Hasse diagram of $\varangle$ but are not in $\triangleleft$.

Claim 2.53. We have $\triangleleft=\triangleleft_{\mathrm{S}}$, so that $\triangleleft \in \operatorname{PFP}(\mathbb{O})$.

The detailed proofs of Claims 2.50 to 2.53 are given in Appendix A.3. This concludes the proof of Proposition 2.49.

We now consider the weak order on $\operatorname{PFP}(\mathbb{O})$. Let us first recall from [14] the definition of the Schröder permutree lattice.

Proposition 2.54 ([14]). Fix an orientation $\mathbb{O}=\left(n, \mathbb{O}^{+}, \mathbb{O}^{-}\right)$of $[n]$.

(1) Each Schröder $\mathbb{O}$-permutree corresponds to a face of the permutreehedron PT $(\mathbb{O})$, and thus to a cone of its normal fan. Moreover, the normal fan of the permutahedron $\operatorname{Perm}(n)$ refines that of the permutreehedron $\mathrm{PT}(\mathbb{O})$. This defines a surjection $\Psi_{\mathbb{O}}$ from the set of ordered partitions of $[n]$ to the set of Schröder permutrees of $\mathrm{SchrPT}(\mathbb{O})$, which sends an ordered partition $\pi$ to the unique Schröder permutree S satisfying that the interior of the normal cone of the face of $\mathrm{PT}(\mathbb{O})$ corresponding to $\mathrm{S}$ contains the interior of the normal cone of the face of $\operatorname{Perm}(n)$ corresponding to $\pi$.

(2) The fibers of this surjection $\Psi_{\mathbb{O}}$ define a lattice congruence of the facial weak order discussed in Section 2.1 .3 (see [14] for details). Therefore, the set of Schröder permutrees $\mathrm{SchrPT}(\mathbb{O})$ is endowed with a lattice structure $\preccurlyeq$, called Schröder permutree lattice, defined by

$$
\mathrm{S} \preccurlyeq \mathrm{S}^{\prime} \Longleftrightarrow \exists \pi, \pi^{\prime} \text { such that } \Psi_{\mathbb{O}}(\pi)=\mathrm{S}, \Psi_{\mathbb{O}}\left(\pi^{\prime}\right)=\mathrm{S}^{\prime} \text { and } \pi \preccurlyeq \pi^{\prime}
$$

(3) The contraction of an edge $e=v \rightarrow w$ in a Schröder permutree $\mathrm{S}$ is called increasing if $\max (p(v))<\min (p(w))$ and decreasing if $\max (p(w))<\min (p(v))$. The Schröder permutree lattice is the transitive closure of the relations $\mathrm{S} \prec \mathrm{S} / e$ (resp. S/e $\prec \mathrm{S}$ ) for any Schröder permutree $\mathrm{S}$ and edge $e \in \mathrm{S}$ defining an increasing (resp. decreasing) contraction.

Now we establish the relationship between the permutree order on $\mathrm{PT}(\mathbb{O})$ and the weak order on $\operatorname{PEP}(\mathbb{O})$.

Proposition 2.55. For any Schröder permutrees $\mathrm{S}, \mathrm{S}^{\prime} \in \operatorname{SchrPT}(\mathbb{O})$, we have $\mathrm{S} \preccurlyeq \mathrm{S}^{\prime}$ in the Schröder permutree lattice if and only if $\triangleleft_{\mathrm{S}} \preccurlyeq \triangleleft_{\mathrm{S}^{\prime}}$ in the weak order on posets.

Proof. We can identify the Schröder $\mathbb{O}$-permutree $\mathrm{S}$ with:

(1) the interval $\left[\mathrm{T}^{\min }(\mathrm{S}), \mathrm{T}^{\max }(\mathrm{S})\right]$ of $\mathbb{O}$-permutrees that belong to the face of $\mathrm{PT}(\mathbb{O})$ given by $\mathrm{S}$,

(2) the interval $\left[\pi^{\min }(\mathrm{S}), \pi^{\max }(\mathrm{S})\right]$ of ordered partitions $\pi$ such that the interior of the normal cone of Perm $(n)$ corresponding to $\pi$ is included in the interior of the normal cone of PT $(\mathbb{O})$ corresponding to $\mathrm{S}$,

(3) the interval $\left[\sigma^{\min }(\mathrm{S}), \sigma^{\max }(\mathrm{S})\right]$ of $\mathfrak{S}(n)$ between the minimal and maximal extensions of $\triangleleft_{S}$.

It is immediate to check that $\sigma^{\min }(\mathrm{S})$ is the minimal linear extension of $\mathrm{T}^{\mathrm{min}}(\mathrm{S})$ and of $\pi^{\min }(\mathrm{S})$ and that $\sigma^{\max }(\mathrm{S})$ is the maximal linear extension of $\mathrm{T}^{\max }(\mathrm{S})$ and 
of $\pi^{\max }(\mathrm{S})$. We conclude that

$$
\begin{aligned}
\mathrm{S} \preccurlyeq \mathrm{S}^{\prime} & \Longleftrightarrow \pi^{\min }(\mathrm{S}) \preccurlyeq \pi^{\min }\left(\mathrm{S}^{\prime}\right) \text { and } \pi^{\max }(\mathrm{S}) \preccurlyeq \pi^{\max }\left(\mathrm{S}^{\prime}\right) \\
& \Longleftrightarrow \sigma^{\min }(\mathrm{S}) \preccurlyeq \sigma^{\min }\left(\mathrm{S}^{\prime}\right) \text { and } \sigma^{\max }(\mathrm{S}) \preccurlyeq \sigma^{\max }\left(\mathrm{S}^{\prime}\right) \\
& \Longleftrightarrow \mathrm{T}^{\min }(\mathrm{S}) \preccurlyeq \mathrm{T}^{\min }\left(\mathrm{S}^{\prime}\right) \text { and } \mathrm{T}^{\max }(\mathrm{S}) \preccurlyeq \mathrm{T}^{\max }\left(\mathrm{S}^{\prime}\right) \Longleftrightarrow \triangleleft_{\mathrm{S}} \preccurlyeq \triangleleft_{\mathrm{S}^{\prime}}
\end{aligned}
$$

The first equivalence holds by definition of the Schröder permutree lattice (since $\left.\Psi_{\mathbb{O}}^{-1}(\mathrm{~S})=\left[\pi^{\min }(\mathrm{S}), \pi^{\max }(\mathrm{S})\right]\right)$, while the last equivalence holds by definition of the weak order on $\operatorname{PFP}(\mathbb{O})\left(\right.$ since $\left.\triangleleft_{\mathrm{S}}=\triangleleft_{\left[\mathrm{T}^{\min }(\mathrm{S}), \mathrm{T}^{\max }(\mathrm{S})\right]}\right)$.

REMARK 2.56. Although the weak order on $\operatorname{PFP}(\mathbb{O})$ is a lattice, the example of Remark 2.11 shows that it is not a sublattice of the weak order on $\mathcal{P}(n)$, nor on $\operatorname{WOIP}(n)$, nor on $\operatorname{PIP}(\mathbb{O})$. We will discuss an alternative description of the meet and join in $\operatorname{PFP}(\mathbb{O})$ in Section 2.4.4.

2.3.5. PIP $(\mathbb{O})$ deletion. Similar to the projection maps of Sections 2.1 .4 and 2.2.4, we

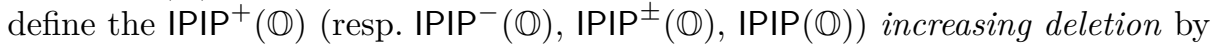

$$
\begin{aligned}
& \triangleleft^{\mathrm{IPIP}^{+}} \operatorname{id}(\mathbb{O}):=\triangleleft \backslash\left\{(a, c) \mid \exists a<b<c, b \in \mathbb{O}^{+} \text {and } a \not b\right\}, \\
& \triangleleft^{\mathrm{IPIP}^{-} \mathrm{id}(\mathbb{O})}:=\triangleleft \backslash\left\{(a, c) \mid \exists a<b<c, b \in \mathbb{O}^{-} \text {and } b \Varangle c\right\}, \\
& \triangleleft^{\mathrm{PIP}^{ \pm} \mathrm{id}(\mathbb{O})}:=\triangleleft \backslash\left\{(a, c) \mid \exists a \leqslant n<p \leqslant c, n \in\{a\} \cup \mathbb{O}^{-}, p \in\{c\} \cup \mathbb{O}^{+} \text {and } n \nless p\right\}, \\
& \triangleleft^{\operatorname{IPIPid}(\mathbb{O})}:=\left(\triangleleft^{\mathrm{IWOIPid}}\right)^{\operatorname{IPIP}^{ \pm} \mathrm{id}(\mathbb{O})},
\end{aligned}
$$

and similarly the $\operatorname{DPIP}^{+}(\mathbb{O})$ (resp. $\left.\operatorname{DPIP}^{-}(\mathbb{O}), \operatorname{DPIP}^{ \pm}(\mathbb{O}), \operatorname{DPIP}(\mathbb{O})\right)$ decreasing deletion by

$$
\begin{aligned}
& \triangleleft^{\mathrm{DPIP}^{+} \mathrm{dd}(\mathbb{O})}:=\triangleleft \backslash\left\{(c, a) \mid \exists a<b<c, b \in \mathbb{O}^{+} \text {and } b \not c\right\}, \\
& \triangleleft^{\mathrm{DPIP}^{-} \mathrm{dd}(\mathbb{O})}:=\triangleleft \backslash\left\{(c, a) \mid \exists a<b<c, b \in \mathbb{O}^{-} \text {and } a \not b\right\}, \\
& \triangleleft^{\mathrm{DPIP}^{ \pm} \mathrm{dd}(\mathbb{O})}:=\triangleleft \backslash\left\{(c, a) \mid \exists a \leqslant p<n \leqslant c, p \in\{a\} \cup \mathbb{O}^{+}, n \in\{c\} \cup \mathbb{O}^{-} \text {and } p \not n\right\}, \\
& \triangleleft^{\operatorname{DPIPdd}(\mathbb{O})}:=\left(\triangleleft^{\mathrm{DWOIPdd}}\right)^{\operatorname{DPIP}^{ \pm} \mathrm{dd}(\mathbb{O})} .
\end{aligned}
$$

These operations are illustrated on Figure 21.

REMARK 2.57. Similar to Remarks 1.10 and 2.15, for any $\varepsilon \in\{\varnothing,-,+, \pm\}$, the $\operatorname{IPIP}^{\varepsilon}(\mathbb{O})$ increasing deletion (resp. $\operatorname{DPIP}^{\varepsilon}(\mathbb{O})$ decreasing deletion) deletes at once all increasing relations which prevent the poset to be in $\operatorname{IPIP}^{\varepsilon}(\mathbb{O})\left(\right.$ resp. in $\left.\operatorname{DPIP}^{\varepsilon}(\mathbb{O})\right)$. Note that we have

$$
\begin{aligned}
& \triangleleft^{\mathrm{IPIP}}{ }^{+} \mathrm{id}(\mathbb{O})=\triangleleft^{\mathrm{IPIP}}{ }^{ \pm} \mathrm{id}\left(\mathbb{O}^{+}, \varnothing\right) \subseteq \triangleleft^{\mathrm{IPIP}^{ \pm}} \mathrm{id}(\mathbb{O}) \\
& \text { and } \quad \triangleleft^{I P I P^{-}} \text {id }(\mathbb{O})=\triangleleft^{I P I P^{ \pm}} \text {id }\left(\varnothing, \mathbb{O}^{-}\right) \subseteq \triangleleft^{I P I P^{ \pm}}{ }^{i d(\mathbb{O})} \text {. }
\end{aligned}
$$

However, we do not necessarily have $\triangleleft^{\mathrm{IPIP}^{ \pm} \mathrm{id}(\mathbb{O})}=\triangleleft^{\mathrm{IPIP}}{ }^{+} \mathrm{id}(\mathbb{O}) \cap \triangleleft^{\mathrm{IPIP}^{-} \mathrm{id}(\mathbb{O})}$. Consider for example the poset $\triangleleft:=\{(1,3),(2,4),(1,4)\}$ and the orientation $(4,\{3\},\{2\})$. Then $\triangleleft \triangleleft^{\mathrm{PIP}^{+} \mathrm{id}(\mathbb{O})}=\{(2,4),(1,4)\}, \quad \triangleleft^{\mathrm{IPIP}^{-} \mathrm{id}(\mathbb{O})}=\{(1,3),(1,4)\}$ so that we have $\triangleleft \mathrm{IPIP}^{+}$id $(\mathbb{O}) \cap \triangleleft^{\mathrm{PIP}^{-}}{ }^{\mathrm{id}(\mathbb{O})}=\{(1,4)\} \neq \varnothing=\triangleleft^{\mathrm{PIP}^{ \pm}} \mathrm{id}(\mathbb{O})$. In other words, we might have to iterate several times the maps $\triangleleft \mapsto \triangleleft^{\mathrm{IPIP}^{+} \mathrm{id}(\mathbb{O})}$ and $\triangleleft \mapsto \triangleleft^{\mathrm{PIP}^{-} \mathrm{id}(\mathbb{O})}$ to obtain the map $\triangleleft \mapsto \triangleleft^{\mathrm{IPIP}^{ \pm}}{ }_{\mathrm{id}(\mathbb{O})}$. This explains the slightly more intricate definition of the map $\triangleleft \mapsto \triangleleft \mathrm{IPIP}^{ \pm} \mathrm{id}(\mathbb{O})$. The same remark holds for the map $\triangleleft \mapsto \triangleleft^{\mathrm{DPIP}^{ \pm} \mathrm{dd}(\mathbb{O})}$.

LEMMA 2.58. For any poset $\triangleleft \in \mathcal{P}(n)$ and any $\varepsilon \in\{\varnothing,-,+, \pm\}$, we have $\triangleleft \operatorname{IPIP}^{\varepsilon} \operatorname{id}(\mathbb{O}) \in \operatorname{IPIP}^{\varepsilon}(\mathbb{O})$ and $\triangleleft^{\operatorname{DPIP}^{\varepsilon} \mathrm{dd}(\mathbb{O})} \in \operatorname{DPIP}^{\varepsilon}(\mathbb{O})$.

Proof. We split the proof into three technical claims whose proofs are given in Appendix A.4. 

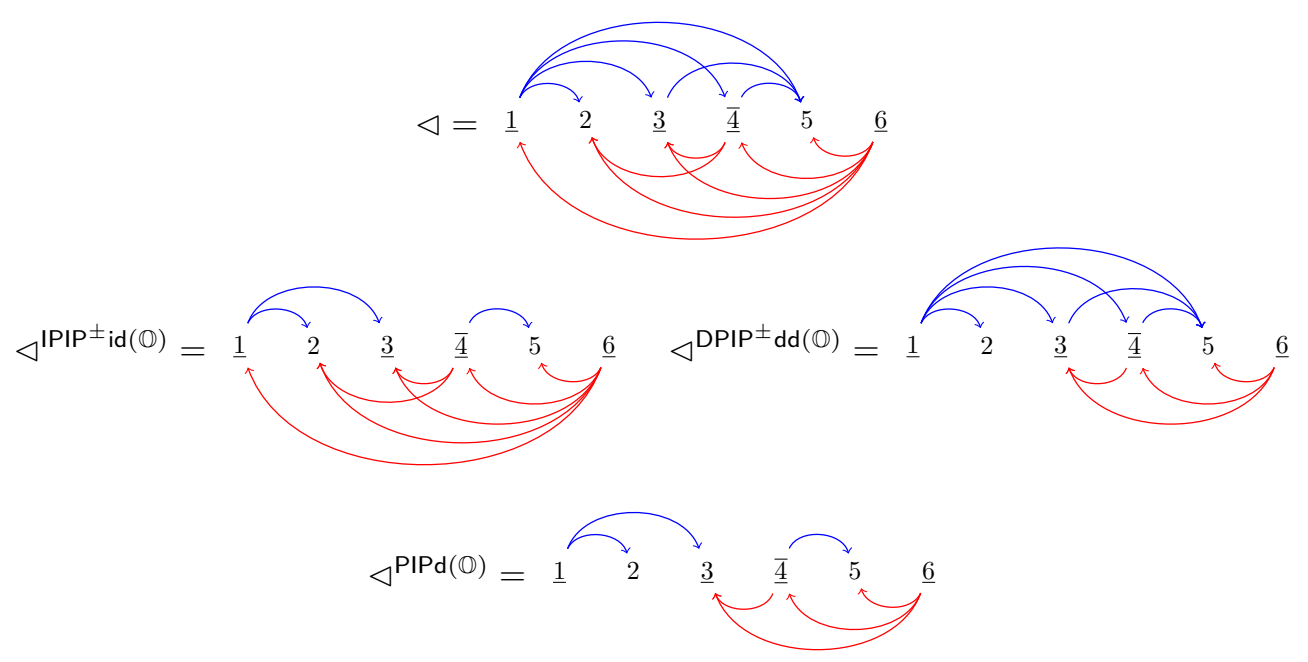

Figure 21. The $\operatorname{IPIP}^{ \pm}(\mathbb{O})$ increasing deletion, the $\operatorname{DPIP}^{ \pm}(\mathbb{O})$ decreasing deletion, and the $\operatorname{PIP}(\mathbb{O})$ deletion.

Claim 2.59. $\triangleleft^{\operatorname{IPIP}{ }^{ \pm} \mathrm{id}(\mathbb{O})}$ is a poset.

Claim 2.60. $\triangleleft^{\mathrm{PIP}^{ \pm} \mathrm{id}(\mathbb{O})}$ is in $\operatorname{IPIP}^{ \pm}(\mathbb{O})$.

This proves the result for $\triangleleft^{\mid P^{P} P^{ \pm} i d(\mathbb{Q})}$. Note that it already contains the result for $\triangleleft^{\mathrm{IPIP}}{ }^{+} \mathrm{id}(\mathbb{O})$, since $\triangleleft^{\mathrm{IIP}^{+}}{ }^{\mathrm{id}(\mathbb{O})}=\triangleleft^{\mathrm{IPIP}}{ }^{ \pm} \mathrm{id}\left(\mathbb{O}^{+}, \varnothing\right) \in \operatorname{IPIP}^{ \pm}\left(\mathbb{O}^{+}, \varnothing\right)=\operatorname{IPIP}^{+}(\mathbb{O})$, and similarly for $\triangleleft^{\mathrm{IPIP}^{-} \mathrm{id}(\mathbb{O})}$.

Claim 2.61. $\triangleleft^{\operatorname{IPIPid}(\mathbb{O})}$ is in $\operatorname{IPIP}(\mathbb{O})$.

Finally, the result for $\triangleleft^{\operatorname{DPIP}^{\varepsilon} \mathrm{dd}(\mathbb{O})}$ with $\varepsilon \in\{\varnothing,-,+, \pm\}$ follows by symmetry.

Lemma 2.62. For any poset $\triangleleft \in \mathcal{P}(n)$ and any $\varepsilon \in\{\varnothing,-,+, \pm\}$, the poset $\triangleleft \operatorname{PIP}^{\varepsilon} \mathrm{id}(\mathbb{O})$ (resp. $\left.\triangleleft^{\mathrm{DPIP}^{\varepsilon} \mathrm{dd}(\mathbb{O})}\right)$ is the weak order minimal (resp. maximal) poset in $\operatorname{IPIP}^{\varepsilon}(\mathbb{O})$ bigger than $\triangleleft\left(\right.$ resp. in $\operatorname{DPIP}^{\varepsilon}(\mathbb{O})$ smaller than $\left.\triangleleft\right)$.

Proof. We prove the result for $\triangleleft^{\mathrm{IPIP}^{\varepsilon} \mathrm{id}(\mathbb{O})}$, the proof for $\triangleleft^{\mathrm{DPIP}^{\varepsilon} \mathrm{dd}(\mathbb{O})}$ being symmetric. Observe that $\triangleleft \preccurlyeq \triangleleft^{\mathrm{PIP}^{\varepsilon} \text { id }(\mathbb{O})}$ since $\triangleleft \mathrm{IPIP}^{\boldsymbol{P}^{\varepsilon}}$ id $(\mathbb{O})$ is obtained from $\triangleleft$ by deleting increasing relations. Consider now $\triangleleft \in \operatorname{IPIP}^{\varepsilon}(\mathbb{O})$ such that $\triangleleft \preccurlyeq \triangleleft$. The following claim is proved in Appendix A.4.

CLAIM 2.63. $\triangleleft^{\operatorname{lnc}} \subseteq\left(\triangleleft^{\operatorname{IPIP} P^{\text {id }}(\mathbb{O})}\right)^{\operatorname{lnc}}$.

This conclude the proof since $\left(\triangleleft^{\operatorname{IPIP} P^{\text {id }}(\mathbb{O})}\right)^{\operatorname{lnc}} \supseteq \triangleleft^{\operatorname{lnc}}$ and $\triangleleft^{\mathrm{Dec}} \subseteq \triangleleft^{\mathrm{Dec}}=$ $\left(\triangleleft \mathrm{IPIP}^{\varepsilon} \text { id }(\mathbb{O})\right)^{\text {Dec }}$ implies that $\triangleleft \mathrm{IPIP}^{\mathrm{S}^{\mathrm{id}}(\mathbb{O})} \preccurlyeq$

Consider now the $\operatorname{PIP}(\mathbb{O})$ deletion defined by

$$
\triangleleft^{\operatorname{PIPd}(\mathbb{O})}:=\left(\triangleleft^{\operatorname{DPIPdd}(\mathbb{O})}\right)^{\operatorname{IPIPid}(\mathbb{O})}=\left(\triangleleft^{\operatorname{IPIPId}(\mathbb{O})}\right)^{\operatorname{DPIPdd}(\mathbb{O})} .
$$

It follows from Lemma 2.58 that $\triangleleft \operatorname{PIPd}(\mathbb{O}) \in \operatorname{PIP}(\mathbb{O})$ for any poset $\triangleleft \in \mathcal{P}(n)$. We now compare this map with the permutree insertion $\Psi_{\mathbb{O}}$ defined in Proposition 2.34.

Proposition 2.64. For any permutation $\sigma \in \mathfrak{S}(n)$, we have $\triangleleft_{\sigma}^{\operatorname{PIPd}(\mathbb{O})}=\triangleleft_{\Psi_{\mathbb{O}}(\sigma)}$. 
Proof. Let $\sigma$ be a permutation of $\mathfrak{S}(n)$ and let $\boldsymbol{\iota}:=\triangleleft_{\sigma}^{\operatorname{PIPd}(\mathbb{O})}$. We already know that $\triangleleft \in \operatorname{PIP}(\mathbb{O})$. The following claim is proved in Appendix A.4.

Claim 2.65. $\triangleleft$ has an $\mathbb{O}$-snake between any two values of $[n]$.

By Proposition 2.44, we thus obtain that $\triangleleft \in \operatorname{PEP}(\mathbb{O})$. Since moreover $\triangleleft_{\sigma}$ is a linear extension of $\boldsymbol{\triangleleft}$, we conclude that $\triangleleft=\triangleleft_{\Psi_{0}(\sigma)}$.

To obtain a similar statement for $\operatorname{WOIP}(n)$, we first need to observe that the map $\triangleleft \mapsto \triangleleft \operatorname{PIPd}(\mathbb{Q})$ commutes with intersections. This straightforward proof is left to the reader.

Proposition 2.66. $(\triangleleft \cap \triangleleft)^{\operatorname{PIPd}(\mathbb{O})}=\triangleleft^{\operatorname{PIPd}(\mathbb{O})} \cap \triangleleft^{\operatorname{PIPd}(\mathbb{O})}$ for any $\triangleleft, \triangleleft \in \mathcal{P}(n)$.

Corollary 2.67. For any permutations $\sigma \preccurlyeq \sigma^{\prime}$, we have $\triangleleft_{\left[\sigma, \sigma^{\prime}\right]}^{\operatorname{PIPd}(\mathbb{O})}=\triangleleft_{\left[\Psi_{\mathbb{Q}}(\sigma), \Psi_{\mathscr{Q}}\left(\sigma^{\prime}\right)\right]}$.

Proof. Applying Propositions 2.64 and 2.66, we obtain

$$
\begin{aligned}
\triangleleft_{\left[\sigma, \sigma^{\prime}\right]}^{\operatorname{PIPd}(\mathbb{C})} & =\left(\triangleleft_{\sigma} \cap \triangleleft_{\sigma^{\prime}}\right)^{\operatorname{PIPd}(\mathbb{O})}=\triangleleft_{\sigma}^{\operatorname{PIPd}(\mathbb{O})} \cap \triangleleft_{\sigma^{\prime}}^{\operatorname{PIPd}(\mathbb{O})} \\
& =\triangleleft_{\Psi_{\odot}(\sigma)} \cap \triangleleft_{\Psi_{0}\left(\sigma^{\prime}\right)}=\triangleleft_{\left[\Psi_{\odot}(\sigma), \Psi_{\odot}\left(\sigma^{\prime}\right)\right] .}
\end{aligned}
$$

Finally, we compare the $\operatorname{PIP}(\mathbb{O})$ deletion with the Schröder permutree insertion defined in Proposition 2.54.

Proposition 2.68. For any ordered partition $\pi$ of $[n]$, we have $\triangleleft_{\pi}^{\operatorname{PIPd}(\mathbb{O})}=\triangleleft_{\Psi_{0}(\pi)}$.

Proof. Let $\pi$ be an ordered partition and let $\triangleleft:=\triangleleft_{\pi}^{\operatorname{PIPd}(\mathbb{Q})}$. We already know that $\triangleleft \in \operatorname{PIP}(\mathbb{O})$. The following claim is proved in Appendix A.4.

Claim 2.69. Any a $<$ c incomparable in $\$ satisfy at least one of the conditions and (\$) of Proposition 2.49.

By Proposition 2.49, we thus obtain that $\triangleleft \in \operatorname{PFP}(\mathbb{O})$. Since moreover any linear extension of $\triangleleft$ extends $\triangleleft_{\pi}$, we conclude that $\triangleleft=\triangleleft_{\Psi_{0}(\pi)}$.

2.4. Sublattices. The previous sections were dedicated to the characterization of various specific families of posets coming from permutreehedra and to the description of the weak order induced by these families. In this final section, we investigate which of these families induce sublattices of the weak order on $\mathcal{P}(n)$. We first introduce some additional notations based on conflict functions which will simplify later the presentation.

2.4.1. Conflict functions. A conflict function is a function of which maps a poset $\triangleleft \in \mathcal{P}(n)$ to a conflict set $\operatorname{cf}(\triangleleft) \subseteq\left(\begin{array}{c}{[n]} \\ 2\end{array}\right)$. A poset $\triangleleft$ is cf-free if $\operatorname{cf}(\triangleleft)=\varnothing$, and we denote the set of cf-free posets on $[n]$ by $\mathcal{F}(\mathrm{cf}, n):=\{\triangleleft \in \mathcal{P}(n) \mid \operatorname{cf}(\triangleleft)=\varnothing\}$. Intuitively, the set $\operatorname{cf}(\triangleleft)$ gathers the conflicting pairs that prevent $\triangleleft$ to be a poset in the family $\mathcal{F}(\mathrm{cf}, n)$.

EXAMPLE 2.70. The characterizations of the families of posets discussed in Sections 2.1, 2.2 and 2.3 naturally translate to conflict functions. For example, the posets in $\operatorname{IWOIP}(n)$ and in $\operatorname{DWOIP}(n)$ are the conflict-free posets for the conflict functions respectively given by

$$
\begin{aligned}
& \operatorname{cf}_{\text {IWOIP }}(\triangleleft)=\{\{a, c\} \mid a \triangleleft c \text { and } \exists a<b<c, \quad a \not b \not c\}, \\
& \operatorname{cf}_{\text {DWOIP }}(\triangleleft)=\{\{a, c\} \mid a \triangleright c \text { and } \exists a<b<c, \quad a \not b \not c\} .
\end{aligned}
$$

The reader can derive from the characterizations of the previous sections other relevant conflict functions. In general, we denote by $\mathrm{cf}_{\mathrm{X}}$ the conflict function defining a family $\mathrm{X}$, i.e. such that $\mathcal{F}\left(\mathrm{cf}_{\mathrm{X}}, n\right)=\mathrm{X}(n)$. 
For a poset $\triangleleft$, we denote by $[\triangleleft]:=\{\{i, j\} \mid i \triangleleft j\} \subseteq\left(\begin{array}{c}{[n]} \\ 2\end{array}\right)$ the support of $\triangleleft$, i.e. the set of pairs of comparable elements in $\triangleleft$. We say that a conflict function of is:

(1) local if $\{a, b\} \in \operatorname{cf}(\triangleleft) \Longleftrightarrow\{a, b\} \in \operatorname{cf}\left(\triangleleft \cap[a, b]^{2}\right)$ for any $a<b$ and any poset $\triangleleft$, i.e. a conflict $\{a, b\}$ only depends on the relations in the interval $[a, b]$,

(2) increasing if $\operatorname{cf}(\triangleleft) \subseteq\left[\triangleleft^{\text {Inc }}\right]$ for any poset $\triangleleft$, i.e. only increasing relations are conflicting; decreasing if $\operatorname{cf}(\triangleleft) \subseteq\left[\triangleleft^{\text {Dec }}\right]$ for any poset $\triangleleft$, i.e. only decreasing relations are conflicting; incomparable if $\operatorname{cf}(\triangleleft) \subseteq\left(\begin{array}{c}{[n]} \\ 2\end{array}\right) \backslash[\triangleleft]$ for any poset $\triangleleft$, i.e. only incomparable pairs are conflicting,

(3) consistent if $\operatorname{cf}(\triangleleft) \cap\left[\triangleleft^{\mathrm{Inc}}\right]=\operatorname{cf}\left(\triangleleft^{\mathrm{Inc}}\right)$ and $\operatorname{cf}(\triangleleft) \cap\left[\triangleleft^{\mathrm{Dec}}\right]=\operatorname{cf}\left(\triangleleft^{\mathrm{Dec}}\right)$ for any poset $\triangleleft$, i.e. increasing (resp. decreasing) conflicts only depends on increasing (resp. decreasing) relations,

(4) monotone if $\triangleleft \subseteq \triangleleft \Longrightarrow \triangleleft \backslash \mathrm{cf}(\triangleleft) \subseteq \varangle \backslash \mathrm{cf}(\triangleleft)$,

(5) semitransitive if $\triangleleft \backslash \operatorname{cf}(\triangleleft)$ is semitransitive, i.e. both increasing and decreasing subrelations of $\triangleleft \backslash \mathrm{cf}(\triangleleft)$ are transitive. In other words, if $a<b<c$ are such that the relations $a \triangleleft b \triangleleft c$ are not conflicts for cf, then the relation $a \triangleleft c$ is not a conflict for of (and similarly for $\triangleright$ ).

EXAMPLE 2.71. The conflict functions $\mathrm{cf}_{\text {IWOIP }}$ and $\mathrm{cf}_{\text {DWOIP }}$ are both local, consistent, monotone and semitransitive. Moreover, $\mathrm{cf}_{\text {IWOIP }}$ is increasing while $\mathrm{cf}_{\text {DWOIP }}$ is decreasing. Indeed, all these properties but the semitransitivity follow directly from the definitions. For the semitransitivity, consider $a<b<c$ with $a \triangleleft b \triangleleft c$ and $\{a, c\} \in \operatorname{cfI}_{\mathrm{IWOIP}}(\triangleleft)$. Then there is $a<d<c$ such that $a \nless d \not c$. Assume for example that $a<d<b$. By transitivity of $\triangleleft$, we have $d \Varangle b$, and thus $\{a, b\} \in \operatorname{cf}_{\text {IWOIP }}(\triangleleft)$.

REMARK 2.72. If $\mathrm{cf}$ and $\mathrm{cf}^{\prime}$ are two conflict functions, then $\mathrm{cf} \cup \mathrm{cf}^{\prime}$ is as well a conflict function with $\mathcal{F}\left(\mathrm{cf} \cup \mathrm{cf}^{\prime}, n\right)=\mathcal{F}(\mathrm{cf}, n) \cap \mathcal{F}\left(\mathrm{cf}^{\prime}, n\right)$. For example, $\mathrm{cf}_{\text {WOIP }}=\mathrm{cf}_{\text {IWOIP }} \cup$ $\mathrm{cf}_{\text {DWOIP }}$ is the conflict function for WOIP $=$ IWOIP $\cap$ DWOIP. Note that all the above conditions are stable by union.

The above conditions suffices to guaranty that cf-free posets induce semi-sublattices of the weak order on $\mathcal{P}(n)$.

Proposition 2.73. For any consistent monotone semitransitive increasing (resp. decreasing) conflict function $\mathrm{cf}$, the set of $\mathrm{cf}$-free posets induces a meet-semi-sublattice of $\left(\mathcal{P}(n), \preccurlyeq, \wedge_{\mathcal{T}}\right)$ (resp. a join-semi-sublattice of $\left(\mathcal{P}(n), \preccurlyeq, \vee_{\mathcal{T}}\right)$ ).

Proof. We prove the result for increasing conflict functions, the proof being symmetric for decreasing ones. Consider two cf-free posets $\triangleleft, \triangleleft$ and their semi-transitive meet $\dashv:=\triangleleft \wedge_{\mathcal{S T}} \triangleleft=\left(\triangleleft^{\text {Inc }} \cup \triangleleft^{\text {Inc }}\right)^{\text {tc }} \cup\left(\triangleleft^{\text {Dec }} \cap \triangleleft^{\text {Dec }}\right)$, so that $\triangleleft \wedge_{\mathcal{T}} \triangleleft=-^{\text {tdd }}$. We want to prove that $\dashv^{\text {tdd }}$ is also cf-free. Assume first that $\dashv$ is not cf-free, and let $\{a, c\} \in \operatorname{cf}(\dashv)$ with $a<c$ and $c-a$ minimal. Since cf is increasing, we have $(a, c) \in \dashv^{\text {Inc }}=\left(\triangleleft^{\operatorname{lnc}} \cup \triangleleft^{\text {Inc }}\right)^{\text {tc }}$. If $(a, c) \notin\left(\triangleleft^{\operatorname{lnc}} \cup \triangleleft^{\text {Inc }}\right)$, then there exists $a=b_{1}<b_{2}<\cdots<b_{k}=c$ such that $a=b_{1} \dashv b_{2} \dashv \cdots \dashv b_{k}=c$. By minimality of $c-a$, all $\left(b_{i}, b_{i+1}\right)$ are in $\dashv \backslash \operatorname{cf}(-)$ while $(a, c)$ is not, which contradicts the semitransitivity of cf. Therefore, $(a, c) \in\left(\triangleleft^{\mathrm{Inc}} \cup \triangleleft^{\mathrm{lnc}}\right)$ and we can assume without loss of generality that $(a, c) \in \triangleleft^{\text {Inc }}$. Since $\triangleleft$ is cf-free and of is consistent, we have $(a, c) \in \triangleleft^{\mathrm{Inc}} \backslash \mathrm{cf}\left(\triangleleft^{\mathrm{Inc}}\right)$. Thus, since of is monotone and $\triangleleft^{\mathrm{Inc}} \subseteq \dashv$, we obtain that $(a, c) \in \dashv \backslash \operatorname{cf}(\dashv)$ which contradicts our assumption that $\{a, c\} \in \operatorname{cf}(\dashv)$. We therefore obtained that $\dashv$ is cf-free. Finally, since of is monotone, consistent, and increasing, and since $\dashv^{\text {Inc }}=\left(\dashv^{\text {tdd }}\right)^{\text {Inc }}$, we conclude that $\dashv^{\text {tdd }}$ is cf-free. 
ExAmple 2.74. Applying Example 2.71 and Proposition 2.73, we obtain that the subposet of the weak order induced by IWOIP $(n)$ (resp. by $\operatorname{DWOIP}(n)$ ) is a meetsemi-sublattice of $\left(\mathcal{P}(n), \preccurlyeq, \wedge_{\mathcal{T}}\right)$ (resp. a join-semi-sublattice of $\left(\mathcal{P}(n), \preccurlyeq, \vee_{\mathcal{T}}\right)$ ), as already proved in Proposition 2.19.

2.4.2. Intervals. We now consider lattice properties of the weak order on permutree interval posets $\operatorname{PIP}(\mathbb{O})$. This section has two main goals:

(1) provide a sufficient condition on $\mathbb{O}$ for $\operatorname{PIP}(\mathbb{O})$ to induce a sublattice of the weak order on $\mathcal{P}(n)$,

(2) show that $\operatorname{PIP}(\mathbb{O})$ induces a sublattice of the weak order on $\operatorname{WOIP}(n)$ for any orientation $\mathbb{O}$.

Using the notations introduced in Section 2.4.1, we consider the conflict functions

$$
\begin{aligned}
& \mathrm{cf}_{\mathrm{IPIP}^{+}(\mathbb{O})}(\triangleleft):=\left\{\{a, c\} \mid a \triangleleft c \text { and } \exists a<b<c, b \in \mathbb{O}^{+} \text {and } a \nless b\right\}, \\
& \mathrm{cf}_{\mathrm{IPIP}^{-}(\mathbb{O})}(\triangleleft):=\left\{\{a, c\} \mid a \triangleleft c \text { and } \exists a<b<c, b \in \mathbb{O}^{-} \text {and } b \Varangle c\right\} \text {, } \\
& \operatorname{cf}_{I P I P I^{ \pm}(\mathbb{O})}(\triangleleft):=\operatorname{cf}_{I_{I P I P}+(\mathbb{O})}(\triangleleft) \cup \operatorname{cf}_{I_{P I P}-(\mathbb{O})}(\triangleleft),
\end{aligned}
$$

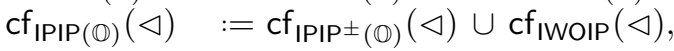

$$
\begin{aligned}
& \mathrm{cf}_{\mathrm{DPIP}^{+}(\mathbb{O})}(\triangleleft):=\left\{\{a, c\} \mid a \triangleright c \text { and } \exists a<b<c, b \in \mathbb{O}^{+} \text {and } b \not c\right\} \text {, } \\
& \mathrm{cf}_{\mathrm{DPIP}^{-}(\mathbb{O})}(\triangleleft):=\left\{\{a, c\} \mid a \triangleright c \text { and } \exists a<b<c, b \in \mathbb{O}^{-} \text {and } a \not b\right\} \text {, } \\
& \mathrm{cf}_{\mathrm{DPIP}^{ \pm}(\mathbb{O})}(\triangleleft):=\mathrm{cf}_{\mathrm{DPIP}^{+}(\mathbb{O})}(\triangleleft) \cup \mathrm{cf}_{\mathrm{DPIP}^{-}(\mathbb{O})}(\triangleleft), \\
& \operatorname{cf}_{\operatorname{DPIP}(\mathbb{O})}(\triangleleft):=\operatorname{cf}_{\operatorname{DPIP}}(\mathbb{O})(\triangleleft) \cup \operatorname{cf}_{\operatorname{DWOIP}}(\triangleleft),
\end{aligned}
$$

and finally

$$
\operatorname{cf}_{\operatorname{PIP}(\mathbb{O})}(\triangleleft):=\operatorname{cf}_{\operatorname{IPIP}(\mathbb{O})}(\triangleleft) \cup \operatorname{cf}_{\operatorname{DPIP}(\mathbb{O})}(\triangleleft)
$$

corresponding to the families studied in Section 2.3.2. As seen in Proposition 2.39, the $\mathrm{cf}_{\mathrm{PIP}(\mathbb{O})}$-free posets are precisely that of $\operatorname{PIP}(\mathbb{O})$.

Covering orientations. In the next statements, we provide a sufficient condition on the orientation $\mathbb{O}$ for $\operatorname{PIP}(\mathbb{O})$ to induce a sublattice of the weak order on $\mathcal{P}(n)$. We first check the conditions of Proposition 2.73 to get semi-sublattices.

LEMMA 2.75. For any orientation $\mathbb{O}$ and any $\varepsilon \in\{\varnothing,-,+, \pm\}$, the conflict functions $\operatorname{cf}_{\mathrm{IPIP}^{\varepsilon}(\mathbb{O})}$ and $\mathrm{cf}_{\mathrm{DPIP}^{\varepsilon}(\mathbb{O})}$ are local, consistent, monotone, and semitransitive. Moreover, $\operatorname{cf}_{\operatorname{IPIP}^{\varepsilon}(\mathbb{O})}$ is increasing while $\operatorname{cf}_{\mathrm{DPIP}^{\varepsilon}(\mathbb{O})}$ is decreasing.

Proof. Since they are stable by union (Remark 2.72), and since they hold for the conflict functions $\mathrm{cf}_{\text {IWOIP }}$ and $\mathrm{cf}_{\text {DWOIP }}$ (Example 2.71), it suffices to show these properties for the conflict functions $\mathrm{cf}_{\mathrm{IPIP}^{+}(\mathbb{O})}, \mathrm{cf}_{\mathrm{IPIP}^{-}(\mathbb{O})}, \mathrm{cf}_{\mathrm{DPIP}^{+}(\mathbb{O})}$ and $\mathrm{cf}_{\mathrm{DPIP}^{-}(\mathbb{O})}$. By

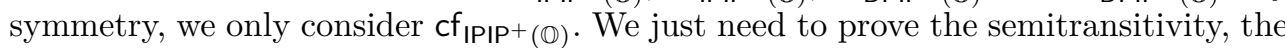
other properties being immediate from the definitions. Consider $a<b<c$ such that $a \triangleleft b \triangleleft c$ and $\{a, c\} \in \operatorname{cf}_{\mathrm{IPIP}^{+}(\mathbb{O})}(\triangleleft)$. Then there exists $a<d<c$ such that $d \in \mathbb{O}^{+}$and $a \nless d$. If $d<b$, then $\{a, b\} \in \operatorname{cf}_{\text {IPIP }^{+}(\mathbb{O})}(\triangleleft)$. Otherwise, $b<d$ and the transitivity of $\triangleleft$ ensures that $b \Varangle d$, so that $\{b, c\} \in \operatorname{cf}_{\operatorname{IPIP}(\mathbb{O})}(\triangleleft)$. We conclude that $\left(\triangleleft \backslash \operatorname{cf}_{I_{I P I}+(\mathbb{O})}(\triangleleft)\right)^{\operatorname{lnc}}$ is transitive. Since $\left(\triangleleft \backslash \operatorname{cf}_{I_{P I P}+(\mathbb{O})}(\triangleleft)\right)^{\text {Dec }}=\triangleleft^{\text {Dec }}$ is also transitive, we obtained that $\mathrm{cf}_{\mathrm{IPIP}^{+}(\mathbb{O})}$ is semitransitive.

Corollary 2.76. For any orientation $\mathbb{O}$ and any $\varepsilon \in\{\varnothing,-,+, \pm\}$, the set $\operatorname{IPIP}^{\varepsilon}(\mathbb{O})$ $\left(\right.$ resp. $\left.\operatorname{DPIP}^{\varepsilon}(\mathbb{O})\right)$ induces a meet-semi-sublattice of $\left(\mathcal{P}(n), \preccurlyeq, \wedge_{\mathcal{T}}\right)$ (resp. a join-semisublattice of $\left.\left(\mathcal{P}(n), \preccurlyeq, \vee_{\mathcal{T}}\right)\right)$.

Proof. Direct application of Lemma 2.75 and Proposition 2.73.

To obtain sublattices, we need an additional condition on $\mathbb{O}$. Namely, we say that an orientation $\mathbb{O}=\left(n, \mathbb{O}^{+}, \mathbb{O}^{-}\right)$is covering if $\{2, \ldots, n-1\} \subseteq \mathbb{O}^{+} \cup \mathbb{O}^{-}$. Note that we 
do not require a priori that $\mathbb{O}^{+} \cap \mathbb{O}^{-}=\varnothing$ nor that $\{1, n\} \subseteq \mathbb{O}^{+} \cup \mathbb{O}^{-}$. Observe also that when $\mathbb{O}$ is covering, we have $\operatorname{IPIP}^{ \pm}(\mathbb{O})=\operatorname{IPIP}(\mathbb{O})$ and $\operatorname{DPIP}^{ \pm}(\mathbb{O})=\operatorname{DPIP}(\mathbb{O})$.

TheOREM 2.77. For any covering orientation $\mathbb{O}$, the sets $\operatorname{IPIP}(\mathbb{O})$, DPIP $(\mathbb{O})$ and $\operatorname{PIP}(\mathbb{O})$ all induce sublattices of the weak order on $\mathcal{P}(n)$.

Proof. We only prove the result for $\operatorname{DPIP}(\mathbb{O})$. It then follows by symmetry for $\operatorname{IPIP}(\mathbb{O})$, which in turn implies the result for $\operatorname{PIP}(\mathbb{O})$ since $\operatorname{PIP}(\mathbb{O})=\operatorname{IPIP}(\mathbb{O}) \cap \operatorname{DPIP}(\mathbb{O})$. We already know from Corollary 2.76 that $\operatorname{DPIP}(\mathbb{O})$ is stable by $\vee_{\mathcal{T}}$ and it remains to show that it is stable by $\wedge_{\mathcal{T}}$. We thus consider two posets $\triangleleft, \triangleleft \in \operatorname{DPIP}(\mathbb{O})$ and let $\dashv:=\triangleleft \wedge_{\mathcal{S T}} \triangleleft=\left(\triangleleft^{\operatorname{lnc}} \cup \triangleleft^{\text {Inc }}\right)^{\text {tc }} \cup\left(\triangleleft^{\text {Dec }} \cap \triangleleft^{\text {Dec }}\right)$, so that $\triangleleft \wedge_{\mathcal{T}} \triangleleft=\dashv^{\text {tdd }}$. We decompose the proof in two steps, whose detailed proofs are given in Appendix A.5.

Claim 2.78. $\dashv$ is in $\operatorname{DPIP}(\mathbb{O})$.

Claim 2.79. $\dashv^{\text {tdd }}$ is in $\operatorname{DPIP}(\mathbb{O})$.

COROLlary 2.80. The weak order on interval posets in the Tamari lattice, in any type $A_{n}$ Cambrian lattice, and in the boolean lattice are all sublattices of the weak order on $\mathcal{P}(n)$.

Proof. Apply Theorem 2.77 to the orientations illustrated in Figure 15: the Tamari lattice is the lattice $\operatorname{PIP}(\varnothing,[n])$, the Cambrian lattices are the lattices $\operatorname{PIP}\left(\mathbb{O}^{+}, \mathbb{O}^{-}\right)$for all partitions $\mathbb{O}^{+} \sqcup \mathbb{O}^{-}=[n]$, and the boolean lattice is the lattice $\operatorname{PIP}([n],[n])$.

REMARK 2.81. The covering condition is essential to the proof of Theorem 2.77. For example, Remark 2.9 shows that $\operatorname{WOIP}(n)=\operatorname{PIP}(\varnothing, \varnothing)$ does not induce a sublattice of the weak order on $\mathcal{P}(n)$.

$\operatorname{PIP}(\mathbb{O})$ induces a sublattice of WOIP $(n)$. We now consider an arbitrary orientation $\mathbb{O}$, not necessarily covering. Although $\operatorname{PIP}(\mathbb{O})$ does not always induce a sublattice of the weak order on $\mathcal{P}(n)$, we show that it always induces a sublattice of the weak order on $\operatorname{WOIP}(n)$.

TheOREM 2.82. For any orientation $\mathbb{O}$ and any $\varepsilon \in\{\varnothing,-,+, \pm\}$, the set $\operatorname{IPIP}^{\varepsilon}(\mathbb{O})$ $\left(\right.$ resp. $\left.\operatorname{DPIP}^{\varepsilon}(\mathbb{O})\right)$ induces a sublattice of the weak order on $\operatorname{WOIP}(n)$.

Proof. By symmetry, it suffices to prove the result for $\operatorname{DPIP}^{-}(\mathbb{O})$. Consider $\triangleleft, \triangleleft \in \mathrm{DPIP}^{-}(\mathbb{O})$. We already know from Corollary 2.76 that $\triangleleft \vee_{\mathcal{T}} \triangleleft \in \operatorname{DPIP}^{-}(\mathbb{O})$. Since $\mathrm{cf}_{\mathrm{DPIP}^{-}(\mathbb{O})}$ is a decreasing conflict function and since the IWOIP increasing deletion only deletes increasing relations, we thus obtain that

$$
\triangleleft V_{\text {WOIP }} \triangleleft=\left(\triangleleft \vee_{\mathcal{T}} \triangleleft\right)^{\text {IWOIPid }} \in \operatorname{DPIP}^{-}(\mathbb{O}) .
$$

It remains to prove that

$$
\triangleleft \wedge_{\text {WOIP }} \triangleleft=\left(\triangleleft \wedge_{\mathcal{T}} \triangleleft\right)^{\text {DWOIPdd }} \in \operatorname{DPIP}^{-}(\mathbb{O}) .
$$

For this, let us denote $\dashv:=\triangleleft \wedge_{\mathcal{S T}} \triangleleft=\left(\triangleleft^{\text {Inc }} \cup \triangleleft^{\text {Inc }}\right)^{\text {tc }} \cup\left(\triangleleft^{\text {Dec }} \cap \triangleleft^{\text {Dec }}\right)$ and $\dashv:=\triangleleft \wedge$ WOIP $\triangleleft$ so that $-\|=\left(\dashv^{\text {tdd }}\right)^{\text {DWOIPdd }}$. As in the proof of Theorem 2.77, we know that $\dashv \in \operatorname{DPIP}^{-}(\mathbb{O})$. Assume now that $-\| \notin \operatorname{DPIP}^{-}(\mathbb{O})$. Consider $\{a, c\} \in \operatorname{cf}_{\operatorname{DPIP}(\mathbb{O})}(-1)$ with $a<c$ and $c-a$ minimal. We therefore have $a \Vdash c$ while there exists $a<b<c$ with $b \in \mathbb{O}^{-}$and $a \| b$. Note that since $\dashv \in \operatorname{DPIP}^{-}(\mathbb{O})$, we have $a \vdash b$. We now distinguish two cases:

- If $a \nvdash^{\text {tdd }} b$, then there exists $i \leqslant b$ and $j \geqslant a$ such that $i \dashv b \dashv a \dashv j$ but $i \rtimes j$. From Lemma 1.15, we know that there exists $a<k<b$ such that $a \nvdash^{\text {tdd }} k \nvdash^{\text {tdd }} b$.

- If $a \vdash^{\text {tdd }} b$, then there exists $a<k_{1}<\cdots<k_{\ell}<b$ such that $a \nvdash^{\text {tdd }} k_{1} \nvdash^{\text {tdd }} \cdots \nvdash^{\text {tdd }} k_{\ell} \nvdash^{\text {tdd }} b$. 
In both cases, there exists $a<k<b$ such that $a \Downarrow k \Downarrow b$. Since $-\| \in$ IWOIP and $a \Vdash c$ while $a \nvdash k$, we must have $k \Vdash c$. But since $k \Downarrow b$, we then have $\{k, c\} \in \operatorname{cf}_{\operatorname{DPIP}(\mathbb{O})}(-\|)$ contradicting the minimality of $c-a$ in our choice of $\{a, c\}$.

COROLlary 2.83. For any orientation $\mathbb{O}$, PIP $(\mathbb{O})$ induces a sublattice of the weak order on $\operatorname{WOIP}(n)$.

Proof. Immediate consequence of Theorem 2.82 as $\operatorname{PIP}(\mathbb{O})=\operatorname{IPIP}(\mathbb{O}) \cap \operatorname{DPIP}(\mathbb{O})$.

2.4.3. Elements. We now consider lattice properties of the weak order on permutree element posets $\operatorname{PEP}(\mathbb{O})$. Similarly to the previous section, the present section has two main goals:

(1) provide a sufficient condition on $\mathbb{O}$ for $\operatorname{PEP}(\mathbb{O})$ to induce a sublattice of the weak order on $\mathcal{P}(n)$,

(2) show that PEP $(\mathbb{O})$ induces a sublattice of the weak order on $\operatorname{WOIP}(n)$ for any orientation $\mathbb{O}$.

We start with a simple observation.

Proposition 2.84. The set $\mathrm{PEP}(\mathbb{O})$ induces a sublattice of the week order on $\mathrm{PIP}(\mathbb{O})$ for any orientation $\mathbb{O}$.

Proof. We have seen in Corollary 2.42 that the meet and join in $\operatorname{PIP}(\mathbb{O})$ are given by

$$
\begin{aligned}
& \triangleleft_{\left[\mathrm{S}, \mathrm{S}^{\prime}\right]} \wedge_{\mathrm{PIP}(\mathbb{O})} \triangleleft_{\left[\mathrm{T}, \mathrm{T}^{\prime}\right]}=\triangleleft_{\left[\mathrm{S} \wedge_{\mathbb{O}} \mathrm{T}, \mathrm{S}^{\prime} \wedge_{\mathbb{O}} \mathrm{T}^{\prime}\right]} \\
& \text { and } \triangleleft_{\left[\mathrm{S}, \mathrm{S}^{\prime}\right]} \vee_{\mathrm{PIP}(\mathbb{O})} \triangleleft_{\left[\mathrm{T}, \mathrm{T}^{\prime}\right]}=\triangleleft_{\left[\mathrm{S} \vee_{\mathbb{O}} \mathrm{T}, \mathrm{S}^{\prime} \vee_{\mathbb{O}} \mathrm{T}^{\prime}\right]} \text {. }
\end{aligned}
$$

Therefore, for any $\mathrm{S}, \mathrm{T} \in \operatorname{PEP}(\mathbb{O})$, we have

$$
\triangleleft_{\mathrm{S}} \wedge_{\mathrm{PIP}(\mathbb{O})} \triangleleft_{\mathrm{T}}=\triangleleft_{[\mathrm{S}, \mathrm{S}]} \wedge_{\mathrm{PIP}(\mathbb{O})} \triangleleft_{[\mathrm{T}, \mathrm{T}]}=\triangleleft_{\left[\mathrm{S} \wedge_{\mathbb{O}} \mathrm{T}, \mathrm{S} \wedge_{\mathbb{O}} \mathrm{T}\right]}=\triangleleft_{\mathrm{S} \wedge_{\mathbb{O}} \mathrm{T}}
$$

Proposition 2.84 enables to show Theorems 2.85 and 2.88 below.

THEOREM 2.85. For any covering orientation $\mathbb{O}, \operatorname{PEP}(\mathbb{O})$ induces a sublattice of the weak order on $\mathcal{P}(n)$.

Proof. PEP $(\mathbb{O})$ induces a sublattice of the weak order on PIP $(\mathbb{O})$ (by Proposition 2.84), which in turn is a sublattice of the weak order on $\mathcal{P}(n)$ when $\mathbb{O}$ is covering (by Theorem 2.77).

Corollary 2.86. The Tamari lattice, any type $A_{n}$ Cambrian lattice, and the boolean lattice are all sublattices of the weak order on $\mathcal{P}(n)$.

Proof. Apply Theorem 2.85 to the orientations illustrated in Figure 15: the Tamari lattice is the lattice $\operatorname{PIP}(\varnothing,[n])$, the Cambrian lattices are the lattices $\operatorname{PIP}\left(\mathbb{O}^{+}, \mathbb{O}^{-}\right)$for all partitions $\mathbb{O}^{+} \sqcup \mathbb{O}^{-}=[n]$, and the boolean lattice is the lattice $\operatorname{PIP}([n],[n])$.

REMARK 2.87. Note that the covering condition in Theorem 2.85 is necessary in general. For example, for the orientation $\mathbb{O}=(5,\{2\},\{4\})$ on $[5]$, the weak order on $\operatorname{PEP}(\mathbb{O})$ is not a sublattice of the weak order on $\mathcal{P}(5)$. For example, for

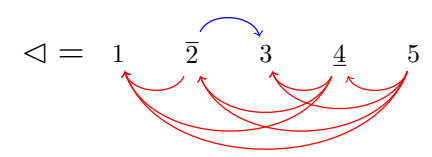

and

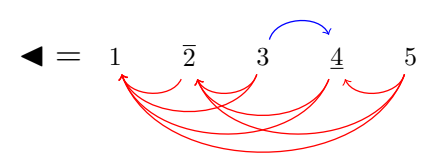

we have 


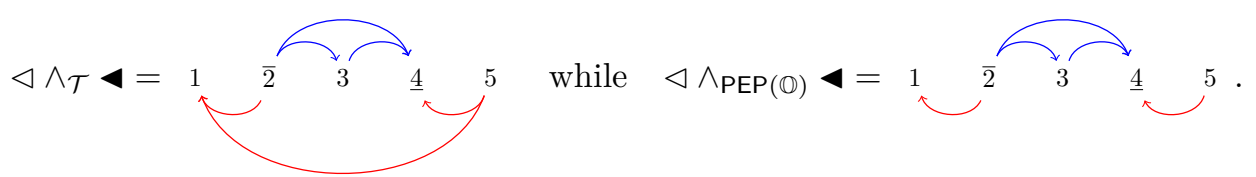

However, for arbitrary orientation, we can still obtain the following weaker statement.

THEOREM 2.88. For any orientation $\mathbb{O}, \operatorname{PEP}(\mathbb{O})$ induces a sublattice of the weak order on $\operatorname{WOIP}(n)$.

Proof. PEP $(\mathbb{O})$ induces a sublattice of the weak order on PIP $(\mathbb{O})$ (by Proposition 2.84), which in turn induces a sublattice of the weak order on WOIP $(n)$ (by Corollary 2.83).

Finally, let us give a poset proof of Proposition 2.84. Recall from Section 2.3.3 and Figure 17 that, for an orientation $\mathbb{O}$ of $[n]$, an $\mathbb{O}$-snake in a poset $\triangleleft$ is a sequence $x_{0}<x_{1}<\cdots<x_{k}<x_{k+1}$ such that

- either $x_{0} \triangleleft x_{1} \triangleright x_{2} \triangleleft x_{3} \triangleright \cdots$ with $\left\{x_{i} \mid i \in[k]\right.$ odd $\} \subseteq \mathbb{O}^{-}$and $\left\{x_{i} \mid i \in[k]\right.$ even $\} \subseteq \mathbb{O}^{+}$,

- or $x_{0} \triangleright x_{1} \triangleleft x_{2} \triangleright x_{3} \triangleleft \cdots$ with $\left\{x_{i} \mid i \in[k]\right.$ odd $\} \subseteq \mathbb{O}^{+}$and $\left\{x_{i} \mid i \in[k]\right.$ even $\} \subseteq \mathbb{O}^{-}$.

Using the notations introduced in Section 2.4.1, we consider the two conflict functions

$$
\begin{gathered}
\operatorname{cf}_{\operatorname{sn}(\mathbb{O})}(\triangleleft):=\{\{a, c\} \mid \text { there is no } \mathbb{O} \text {-snake joining } a \text { to } c\}, \\
\operatorname{cf}_{\operatorname{PEP}(\mathbb{O})}(\triangleleft):=\operatorname{cf}_{\operatorname{PIP}(\mathbb{O})}(\triangleleft) \cup \mathrm{cf}_{\operatorname{sn}(\mathbb{O})}(\triangleleft) .
\end{gathered}
$$

As seen in Proposition 2.44, $\mathrm{cf}_{\mathrm{sn}(\mathbb{O})}$ corresponds to the condition characterizing $\operatorname{PEP}(\mathbb{O})$ in $\operatorname{PIP}(\mathbb{O})$, so that the $\mathrm{cf}_{\mathrm{PEP}(\mathbb{O})}$-free posets are precisely that of $\operatorname{PEP}(\mathbb{O})$. We now prove that the conflict function $\mathrm{cf}_{\mathrm{sn}(\mathbb{O})}$ alone induces a sublattice of the weak order on posets.

Proposition 2.89. For any orientation $\mathbb{O}$ on $[n]$, the set of $\mathrm{cf}_{\mathrm{sn}(\mathbb{O})}$-free posets induces a sublattice of the weak order on $\mathcal{P}(n)$.

Proof. Consider two $\mathrm{cf}_{\mathrm{sn}(\mathbb{O})}$-free posets $\triangleleft, \triangleleft$ and consider their semi-transitive meet $\dashv:=\triangleleft \wedge_{\mathcal{S T}} \triangleleft=\left(\triangleleft^{\mathrm{Inc}} \cup \triangleleft^{\text {Inc }}\right)^{\text {tc }} \cup\left(\triangleleft^{\mathrm{Dec}} \cap \triangleleft^{\mathrm{Dec}}\right)$, so that $\triangleleft \wedge_{\mathcal{T}} \triangleleft=\dashv^{\text {tdd }}$. As in the proof of Theorem 2.77, we decompose the proof in two steps, whose detailed proofs are given in Appendix A.6.

Claim 2.90. $\dashv i s \mathrm{cf}_{\mathrm{sn}(\mathbb{O})}-$ free.

Claim 2.91. $\dashv^{\text {tdd }}$ is $\mathrm{cf}_{\mathrm{sn}(\mathbb{O})}$-free.

Note that Proposition 2.89 provides a proof of Proposition 2.84 on posets. It also enables us to obtain further results for the specific orientation $(n, \varnothing, \varnothing)$. Indeed, Proposition 2.1 ensures that the $\mathrm{cf}_{\mathrm{sn}(\mathbb{O})^{-}}$free posets are precisely the posets of $\operatorname{WOEP}(n)$. Proposition 2.89 therefore specializes to the following statement.

COROLlary 2.92. The set $\operatorname{WOEP}(n)$ induces a sublattice of the weak order on $\mathcal{P}(n)$.

2.4.4. Faces. In this section, we study the lattice properties of the weak order on permutree face posets PFP $(\mathbb{O})$. We have seen in Propositions 2.54 and 2.55 that the weak order on $\operatorname{PFP}(\mathbb{O})$ coincides with the Schröder permutree lattice, but we have observed in Remark 2.56 that it is not a sublattice of the weak order on $\mathcal{P}(n)$, nor on $\operatorname{WOIP}(n)$, nor on $\operatorname{PIP}(\mathbb{O})$. For completeness, let us report on a method to compute 
the meet and join directly on the posets of $\operatorname{PFP}(\mathbb{O})$. For that, define the $\operatorname{PFP}(\mathbb{O})$ increasing addition and the PFP $(\mathbb{O})$ decreasing addition by

$$
\triangleleft^{\mathrm{PFPia}}= \begin{cases}\triangleleft & \text { if } \triangleleft \in \operatorname{PFP}(\mathbb{O}) \\ (\triangleleft \cup\{(a, c) \mid a<c \text { not satisfying }(\boldsymbol{A}) \text { nor }(\boldsymbol{a})\})^{\mathrm{PFPia}} & \text { otherwise }\end{cases}
$$

and

$$
\triangleleft^{\mathrm{PFPda}}= \begin{cases}\triangleleft & \text { if } \triangleleft \in \mathrm{PFP}(\mathbb{O}) \\ (\triangleleft \cup\{(c, a) \mid a<c \text { not satisfying }(\boldsymbol{A}) \text { nor }(\boldsymbol{中})\})^{\mathrm{PFPda}} & \text { otherwise. }\end{cases}
$$

Experimental observations indicate that for $\mathrm{S}^{\prime} \mathrm{S}^{\prime} \in \operatorname{PFP}(\mathbb{O})$,

$$
\mathrm{S} \wedge_{\mathrm{PFP}(\mathbb{O})} \mathrm{S}^{\prime}=\left(\mathrm{S} \wedge_{\text {WOIP }} \mathrm{S}^{\prime}\right)^{\text {PFPia }} \quad \text { and } \quad \mathrm{S} \vee_{\mathrm{PFP}(\mathbb{O})} \mathrm{S}^{\prime}=\left(\mathrm{S} \vee_{\text {WOIP }} \mathrm{S}^{\prime}\right)^{\text {PFPda }}
$$

A complete proof of this observation would however be quite technical. It would in particular require a converging argument to prove that the $\operatorname{PFP}(\mathbb{O})$ increasing and decreasing additions are well defined.

\section{Appendix A. Missing Claims}

This appendix gathers all missing proofs of the technical claims used in Section 2.

\section{A.1. Proof of Claims of Section 2.1.4.}

Proof of Claim 2.13. First, $\triangleleft^{\text {maxle }}$ is clearly antisymmetric since it is obtained from an antisymmetric relation by adding just decreasing relations between some incomparable elements. To prove that $\triangleleft^{\text {maxle }}$ is transitive, consider $u, v, w \in[n]$ be such that $u \triangleleft^{\text {maxle }}$ $v \triangleleft^{\text {maxle }} w$. We distinguish four cases:

(1) If $u \rtimes v \rtimes w$, then we have $w<v<u$ with $w \Varangle v \rtimes u$. Our assumption thus ensures that $w \Varangle u$. Thus, either $u \triangleleft w$ or $u$ and $w$ are incomparable. In both cases, $u \triangleleft^{\text {maxle }} w$.

(2) If $u \Varangle v \triangleleft w$, then we have $v<u$ with $v \rtimes u$. We then have two cases:

- Assume that $u<w$. Since $v<u<w$ and $v \Varangle u$ while $v \triangleleft w$, our assumption implies that $u \triangleleft w$, so that $u \triangleleft^{\text {maxle }} w$.

- Assume that $w<u$. Since $v \Varangle u$ and $v \triangleleft w$, the transitivity of $\triangleleft$ impose that $w \Varangle u$. Thus either $u \triangleleft w$ or $u$ and $w$ are incomparable. In both cases, $u \triangleleft^{\text {maxle }} w$.

(3) If $u \triangleleft v \pitchfork w$, then we have $w<v$ with $w \Varangle v$. We then have two cases:

- Assume that $u<w$. Since $u<w<v$ and $w \Varangle v$ while $u \triangleleft v$, our assumption implies that $u \triangleleft w$ so that $u \triangleleft^{\text {maxle }} w$.

- Assume that $w<u$. Since $w \Varangle v$ and $u \triangleleft v$, the transitivity of $\triangleleft$ impose that $w \rtimes u$. Thus, either $u \triangleleft w$ or $u$ and $w$ are incomparable. In both cases, $u \triangleleft^{\text {maxle }} w$.

(4) If $u \triangleleft v \triangleleft w$, then $u \triangleleft w$ by transitivity of $\triangleleft$ and thus $u \triangleleft^{\text {maxle }} w$.

We proved in all cases that $u \triangleleft^{\text {maxle }} w$, so that $\triangleleft$ is transitive. Since all our relations are reflexive, we conclude that $\triangleleft^{\text {maxle }}$ is a poset.

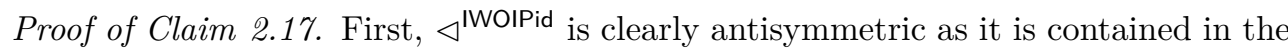
antisymmetric relation $\triangleleft$. To prove that it is transitive, consider $u, v, w \in[n]$ such that $u \triangleleft^{\text {IWOIPid }} v \triangleleft^{\text {IWOIPid }} w$. Since $\triangleleft^{\text {IWOIPid }} \subseteq \triangleleft$, we have $u \triangleleft v \triangleleft w$, so that $u \triangleleft w$ by transitivity of $\triangleleft$. Assume by means of contradiction that $u \not^{\text {IWOIPid }} w$. Thus, $u<w$ and there exists $u<z_{1}<\cdots<z_{k}<w$ such that $u \pitchfork z_{1} \Varangle \cdots \pitchfork z_{k} \pitchfork w$. We now distinguish three cases:

(1) If $v<u$, then $v \not_{\text {IWOIPid }} w$ since $v<u<z_{1}<\cdots<z_{k}<w$ and $v \rtimes u \pitchfork z_{1} \rtimes \cdots \pitchfork z_{k} \rtimes w$. 
(2) If $u<v<w$, consider $\ell \in[k]$ such that $z_{\ell} \leqslant v<z_{\ell+1}$ (with $\ell=0$ if $v<z_{1}$ and $\ell=k$ if $z_{k} \leqslant v$ ). Since $z_{\ell} \not z_{\ell+1}$ and $\triangleleft$ is transitive, we have either $z_{\ell} \nless v$ or $v \nless z_{\ell+1}$. In the former case, we have $u \Varangle_{\text {IWOIPid }} v$ since $u<z_{1}<\cdots<z_{\ell} \leqslant v$ and $u \pitchfork z_{1} \not \cdots \pitchfork z_{\ell} \pitchfork v$. In the latter case, we have $v \Varangle^{\text {IWOIPid }} w$ since $v<z_{\ell+1}<\cdots<z_{k}<w$ and $v \Varangle z_{\ell+1} \not \cdots \rtimes z_{k} \nrightarrow w$.

(3) If $w<v$, then $u \not^{\text {IWOIPid }} v$ since $u<z_{1}<\cdots<z_{k}<w<v$ and $u \pitchfork z_{1} \pitchfork \cdots \rtimes z_{k} \nless w \pitchfork w$.

As we obtained a contradiction in each case, we conclude that $\triangleleft^{\text {IWOIPid }}$ is transitive. Since all our relations are reflexive, we conclude that $\triangleleft^{\text {IWOIPid }}$ is a poset.

\section{A.2. Proof of Claims of Proposition 2.44}

Proof of Claim 2.45. By symmetry, we only need to prove the first statement. Note that $u$ and $w$ are incomparable, otherwise $u \triangleleft v$ and $v \triangleright w$ could not both be cover relations. Therefore, there is a non-degenerate $\mathbb{O}$-snake $u=x_{0}<x_{1}<\cdots<$ $x_{k}<x_{k+1}=w$ from $u$ to $w$. Assume first that $x_{1}<v$. If $x_{1} \in \mathbb{O}^{+}$and $u \triangleright x_{1}$, then $u \triangleleft v, x_{1} \in \mathbb{O}^{+}$and $\triangleleft \in \operatorname{IPIP}^{+}(\mathbb{O})$ implies that $u \triangleleft x_{1}$, a contradiction. If $x_{1} \in \mathbb{O}^{-}$and $u \triangleleft x_{1}$, then $u \triangleleft v, x_{1} \in \mathbb{O}^{-}$and $\triangleleft \in \operatorname{IPIP}^{+}(\mathbb{O})$ implies that $x_{1} \triangleleft v$ which together with $u \triangleleft x_{1}$ would contradict that $u \triangleleft v$ is a cover relation. As we reach a contradiction in both cases, we obtain that $v \leqslant x_{1}$, and by symmetry $x_{k} \leqslant v$. Therefore, we have $x_{1}=v=x_{k}$, so that $u<v<w$ and $v \in \mathbb{O}^{-}$.

Proof of Claim 2.46. We work by induction on $p$, the case $p=1$ being immediate. By symmetry, we can assume that $x_{0} \in \mathbb{O}^{-}, x_{0} \triangleright x_{1}$ and $x_{0}<x_{1}$. Let $j$ be the first position in the path such that $x_{j-1} \triangleright x_{j} \triangleleft x_{j+1}$ (by convention $j=p$ if $\left.x_{0} \triangleright x_{1} \triangleright \ldots \triangleright x_{p}\right)$. Assume that there is $i \in[j]$ such that $x_{i} \leqslant x_{0}$, and assume that $i$ is the first such index. Since $x_{i} \leqslant x_{0}<x_{i-1}, x_{i} \triangleleft x_{i-1}, x_{0} \in \mathbb{O}^{-}$and $\triangleleft \in \operatorname{IPIP}^{-}(\mathbb{O})$, we obtain $x_{0} \triangleleft x_{i-1}$, a contradiction. This shows that $x_{0}<x_{i}$ for $i \in[j]$. If $j=p$, the statement is proved. Otherwise, we consider $x_{j-1}, x_{j}$ and $x_{j+1}$. By Claim 2.45, we have $x_{j} \in \mathbb{O}^{+}$and either $x_{j-1}<x_{j}<x_{j+1}$ or $x_{j+1}<x_{j}<x_{j-1}$. In the latter case, $x_{0}<x_{j}<x_{j-1}, x_{0} \triangleright x_{j-1}, x_{j} \in \mathbb{O}^{+}$and $\triangleleft \in \operatorname{DPIP}^{+}(\mathbb{O})$ would imply $x_{j} \triangleright x_{j-1}$, a contradiction. We thus obtain that $x_{j} \in \mathbb{O}^{+}, x_{j} \triangleleft x_{j+1}$ and $x_{j}<x_{j+1}$. The induction hypothesis thus ensures that $x_{j}<x_{i}$ for all $j<i \leqslant p$. This concludes since $x_{0}<x_{j}$.

\section{A.3. Proof of Claims of Proposition 2.49.}

Proof of Claim 2.50. By Proposition 2.44, we just need prove that there is an $\mathbb{O}$-snake between any two values of $[n]$. Otherwise, consider $a<c$ with $c-a$ minimal such that there is no $\mathbb{O}$-snake between $a$ and $c$. In particular, $a$ and $c$ are incomparable. By $(\boldsymbol{A})$, we can assume for instance that there is $a<b<c$ such that $b \in \mathbb{O}^{-}$and $a \not b \nless c$. By minimality of $c-a$, there is an $\mathbb{O}$-snake $a=x_{0}<x_{1}<\cdots<x_{k}<x_{k+1}=b$. Then we have either $x_{1} \in \mathbb{O}^{-}$and $a \triangleleft x_{1}$, or $x_{1} \in \mathbb{O}^{+}$and $a \triangleright x_{1}$ (note that this holds even when $x_{1}=b$ since $a \not b$ and $b \in \mathbb{O}^{-}$). Moreover, by minimality of $c-a$, there is an $\mathbb{O}$-snake between $x_{1}$ and $c$. Lemma 2.47 thus ensures that there is as well an $\mathbb{O}$-snake between $a$ and $c$, contradicting our assumption.

Proof of Claim 2.51. By definition, we have $\triangleleft \subseteq \varangle$. Assume now that $\triangleleft^{\text {Dec }} \neq \triangleleft^{\text {Dec }}$ and let $x<y$ be such that $x \not y$ but $x \triangleright y$. By definition of $\boldsymbol{\iota}$, there exists a minimal path $y=z_{0}, z_{1}, \ldots, z_{k}=x$ such that for all $i \in[k]$, either $z_{i-1} \triangleleft z_{i}$, or $z_{i-1}<z_{i}$ are incomparable in $\triangleleft$ and do not satisfy ( ) . Since $x \not y$ and $x<y$, we have $k \geqslant 2$ and there exists $i \in[k-1]$ such that $z_{i+1}<z_{i-1}$. We distinguish three cases: 
- If $z_{i}<z_{i+1}<z_{i-1}$, then $z_{i} \triangleright z_{i-1}$ and $z_{i} \not z_{i+1}$, and thus $z_{i+1} \triangleright z_{i-1}$ as $\triangleleft \in \operatorname{DWOIP}(n)$.

- If $z_{i+1}<z_{i}<z_{i-1}$, then $z_{i+1} \triangleright z_{i} \triangleright z_{i-1}$ and thus $z_{i+1} \triangleright z_{i-1}$ by transitivity.

- If $z_{i+1}<z_{i-1}<z_{i}$, then $z_{i+1} \triangleright z_{i}$ and $z_{i-1} \not z_{i}$, and thus $z_{i+1} \triangleright z_{i-1}$ as $\triangleleft \in \operatorname{DWOIP}(n)$.

In all cases, $z_{i+1} \triangleright z_{i-1}$ contradicts the minimality of the path.

Proof of Claim 2.52. We first show that $4 \in \mathrm{PIP}(\mathbb{O})$. Since $\triangleleft^{\mathrm{Dec}}=\varangle^{\mathrm{Dec}}$ and $\triangleleft \in \operatorname{DPIP}(\mathbb{O})$, we have $\varangle \in \operatorname{DPIP}(\mathbb{O})$ and we just need to show that $\varangle \in \operatorname{IPIP}(\mathbb{O})$. Consider thus $a<b<c$ such that $a \varangle c$. By definition of $\varangle$, there exists $a^{\prime}<b<c^{\prime}$ such that $a \triangleleft a^{\prime}, c^{\prime} \triangleleft c$, and either $a^{\prime} \triangleleft c^{\prime}$, or $a^{\prime}$ and $c^{\prime}$ are incomparable in $\triangleleft$ and do not satisfy $(\mathbf{P})$. We now proceed in two steps:

(1) Our first goal is to show that either $a^{\prime} \varangle b$ or $b \triangleleft c^{\prime}$ which by transitivity shows that $(a, c)$ satisfies the WOIP condition. Assume that $a^{\prime} b c^{\prime}$. The transitivity of $\boldsymbol{\varangle}$ ensures that both pairs $a^{\prime}, b$ and $b, c^{\prime}$ are incomparable in 4 . Therefore, they are incomparable in $\triangleleft$ and satisfy ( $)$. Let us focus on $a^{\prime}, b$. Assume first that there is $a^{\prime}<d<b$ such that $d \in \mathbb{O}^{+}$and $a^{\prime} \Varangle d \not b$. Since $\triangleleft \in \operatorname{IPIP}(\mathbb{O})$, we cannot have $a^{\prime}<d<c^{\prime}, d \in \mathbb{O}^{+}, a^{\prime} \Varangle d$ and $a^{\prime} \triangleleft c^{\prime}$. Therefore, $a^{\prime}$ and $c^{\prime}$ do not satisfy $(\boldsymbol{A})$, which together with $a^{\prime} \not d$ implies that $d \triangleright c^{\prime}$. We obtain $d<b<c^{\prime}$ with $d \not b \not c^{\prime}$ while $d \triangleright c^{\prime}$ contradicting that $\triangleleft \in \operatorname{DWOIP}(n)$. Assume now that there is $a^{\prime}<d<b$ such that $d \in \mathbb{O}^{-}$ and $a^{\prime} \not d \nless b$. If $a^{\prime} \triangleleft c^{\prime}$, then $d \triangleleft c^{\prime}$ since $a^{\prime}<d<c^{\prime}, d \in \mathbb{O}^{-}$and $\triangleleft \in \operatorname{IPIP}(\mathbb{O})$. If $a^{\prime}$ and $c^{\prime}$ do not satisfy $(\mathbf{(}), d \in \mathbb{O}^{-}$and $a^{\prime} \not d$ imply $d \triangleleft c^{\prime}$. In both cases, we obtain $d<b<c^{\prime}$ with $d \not b \not c^{\prime}$ while $d \triangleleft c^{\prime}$ contradicting that $\triangleleft \in \operatorname{IWOIP}(n)$. Since we reach a contradiction in all cases, we conclude that $a^{\prime} \triangleleft b$ or $b \triangleleft c^{\prime}$.

(2) We now want to check that the orientation constraint on $b$ is also satisfied. Assume $b \in \mathbb{O}^{+}$. If $a^{\prime} \triangleleft c^{\prime}$, we have $a^{\prime} \triangleleft b$ since $\triangleleft \in \operatorname{IPIP}(\mathbb{O})$, and thus $a^{\prime} \triangleleft b$ since $\triangleleft \subseteq \varangle$. If $a^{\prime}$ and $c^{\prime}$ do not satisfy ( $)$, then $a^{\prime} \triangleleft b$ or $b \triangleright c^{\prime}$, which

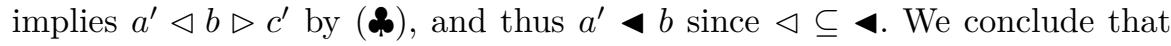
$b \in \mathbb{O}^{+} \Rightarrow a^{\prime} \varangle b$ and by symmetry that $b \in \mathbb{O}^{-} \Rightarrow b \varangle c^{\prime}$.

Since $a \varangle a^{\prime}, c^{\prime} \varangle c$ and $\varangle$ is transitive, we obtain that $a \varangle b$ or $b \triangleleft c$, and that $b \in \mathbb{O}^{+} \Rightarrow a \triangleleft b$ and $b \in \mathbb{O}^{-} \Rightarrow b \triangleleft c$. We conclude that $\varangle \in \operatorname{PIP}(\mathbb{O})$.

There is left to prove that any $a<c$ incomparable in $\varangle$ satisfy (\$). Assume the opposite and consider $a<c$ incomparable in $\varangle$ not satisfying $(\boldsymbol{(})$ with $c-a$ minimal. Since $a$ and $c$ are incomparable in $\triangleleft$, they are also incomparable in $\triangleleft$ and satisfy $(\boldsymbol{\Lambda})$. Assume for example that there exists $b \in \mathbb{O}^{+}$such that $a \not b \not c$ (the other case is symmetric). Since $\triangleleft^{\text {Dec }}=\varangle^{\text {Dec }}$, we have $b \downarrow c$. Since $a$ and $c$ do not satisfy $(\boldsymbol{\uparrow})$ in $\varangle$, we obtain that $a \triangleleft b$. We can assume that $b$ is the maximal integer such that $a<b<c$, $b \in \mathbb{O}^{+}$and $a \triangleleft b \downarrow c$. Since $a \triangleleft b$ but $a \backslash c$, we have $b \backslash c$, so that $b$ and $c$ are incomparable in 4 . By minimality of $c-a$, we obtain that $b$ and $c$ satisfy $(\boldsymbol{\$})$ in 4 . We distinguish two cases:

(1) Assume that there exists $b<d<c$ such that $d \in \mathbb{O}^{+}$and $b \backslash d \downarrow c$. Since $a$ and $c$ do not satisfy $(\boldsymbol{\leftrightarrow})$ in $\boldsymbol{\triangleleft}$, we have $a \triangleleft d \downarrow c$, contradicting the maximality of $b$.

(2) Assume that there exists $b<d<c$ such that $d \in \mathbb{O}^{-}$and $b \downarrow d<c$.

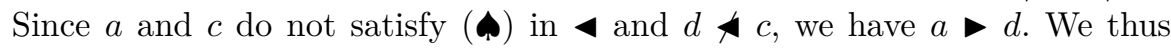
obtained $a<b<d$ with $b \in \mathbb{O}^{+}$, and $a \triangleright d$ while $b \downarrow d$ contradicting that $\varangle \in \operatorname{PIP}(\mathbb{O})$.

Since we obtain a contradiction in both cases, we conclude that any $a<c$ incomparable in $\triangleleft$ satisfy $(\boldsymbol{\Lambda})$. 
Proof of Claim 2.53. We first prove that $\triangleleft \subseteq \triangleleft_{\mathrm{S}}$. Observe first that for a permutree $\mathrm{T}$ and a Schröder permutree $\mathrm{S}$ obtained from $\mathrm{T}$ by contracting a subset of edges $E$, the poset $\triangleleft_{\mathrm{S}}$ is obtained from the poset $\triangleleft_{\mathrm{T}}$ by deleting the sets

$$
\begin{gathered}
\left\{(a, d) \mid \begin{array}{c}
a<d, \exists a \leqslant b<c \leqslant d, b \in\{a\} \cup \mathbb{O}^{-}, c \in\{d\} \cup \mathbb{O}^{+}, \\
a \triangleleft_{\mathrm{T}} b \triangleleft_{\mathrm{T}} c \triangleleft_{\mathrm{T}} d \text { and }(b, c) \in E
\end{array}\right\}, \\
\left\{(d, a) \mid \begin{array}{c}
a<d, \exists a \leqslant b<c \leqslant d, b \in\{a\} \cup \mathbb{O}^{+}, c \in\{d\} \cup \mathbb{O}^{-}, \\
a \triangleright_{\mathrm{T}} b \triangleright_{\mathrm{T}} c \triangleright_{\mathrm{T}} d \text { and }(c, b) \in E
\end{array}\right\} .
\end{gathered}
$$

Assume now that we had $\triangleleft \nsubseteq \triangleleft_{\mathrm{S}}$ and remember that $\triangleleft \subseteq \triangleleft_{\mathrm{S}}$ by construction. Since we only contract increasing edges in $\triangleleft_{\mathrm{T}}$ to obtain $\triangleleft_{\mathrm{S}}$, this would imply that there exists $a \leqslant b<c \leqslant d$ with $b \in\{a\} \cup \mathbb{O}^{-}, c \in\{d\} \cup \mathbb{O}^{+}$, and such that $a \triangleleft d$ while $b \Varangle d$. This would contradict that $\triangleleft \in \operatorname{PIP}(\mathbb{O})$.

We now prove that $\triangleleft_{\mathrm{S}} \subseteq \triangleleft$. Observe first that $\triangleleft_{\mathrm{S}}^{\mathrm{Dec}} \subseteq \triangleleft^{\text {Dec }}=\triangleleft^{\text {Dec }}$. Assume now by contradiction that there exists $a<c$ such that $a \Varangle c$ and $a \triangleleft_{\mathrm{S}} c$, and choose such $a<c$ with $c-a$ minimal. Note that $a$ and $c$ are incomparable in $\triangleleft$. We distinguish two cases:

(1) If $a$ and $c$ satisfy ( $(\mathbf{s})$, we can assume by symmetry that there exists $a<b<c$ such that $b \in \mathbb{O}^{+}$and $a \nless b \not c$. Since $a<b<c, b \in \mathbb{O}^{+}, a \triangleleft_{\mathrm{S}} c$ and $\triangleleft_{\mathrm{S}} \in \mathrm{PIP}(\mathbb{O})$, we obtain that $a \triangleleft_{\mathrm{S}} b$. Since $a \rtimes b$ and $a \triangleleft_{\mathrm{S}} b$, this contradicts the minimality of $c-a$.

(2) If $a$ and $c$ do not satisfy (\$), then they satisfy (\$), and $a \boldsymbol{4}$ is not a cover relation (otherwise, the relation $a \varangle c$ would have been contracted in $\triangleleft_{\mathrm{S}}$ ). Let $b \in[n] \backslash\{a, c\}$ be such that $a \triangleleft b \triangleleft c$. If $a<b<c$, we have $a \not b \not c$, thus $a \Varangle b \Varangle c$ by $(\mathbf{a})$, thus $a \Varangle_{\mathrm{S}} b \Varangle_{\mathrm{S}} c$ by minimality of $c-a$, contradicting that $a \triangleleft_{\mathrm{S}} c$ and $\triangleleft_{\mathrm{S}} \in \operatorname{WOIP}(n)$. We can thus consider that $b<a<c$ (the case $a<c<b$ is symmetric). Note that we cannot have $a \in \mathbb{O}^{+}$since $b$ and $b \triangleleft c$ would contradict that $\varangle \in \operatorname{PIP}(\mathbb{O})$. We have $b \triangleright a($ since $b \triangleright a)$ and we can assume that $b$ is maximal such that $b<a$ and $b \triangleright a$. Observe that $b$ and $c$ are incomparable in $\triangleleft$ (indeed $b \Varangle c$ since $b \triangleright a$ and $a \nless c$, and $b \not c$ since $b \triangleleft c)$. Since $b<a<c$ and $b \triangleright a$ while $a \not c, b$ and $c$ do not satisfy (\$), thus they satisfy $(\boldsymbol{\beta})$. We again have two cases:

- If there is $b<d<c$ with $d \in \mathbb{O}^{+}$with $b \nless d \not c$. If $b<d<a$, we have $b \triangleright a$ and $\triangleleft \in \operatorname{PIP}(\mathbb{O})$ implies $d \triangleright a$ contradicting the maximality of $b$. Since $d \in \mathbb{O}^{+}$, we have $d \neq a$. Finally, if $a<d<c$, we have $a \not d$ since $d \not c$ and $a$ and $c$ satisfy (\$), and we obtain that $a \nless d \not c$ contradicting that $a$ and $c$ do not satisfy $(\boldsymbol{A})$.

- If there is $b<d<c$ with $d \in \mathbb{O}^{-}$with $b \not d \not c$, then we have $a<d<c$ (because $b \not d, b \triangleright a$ and $\triangleleft \in \operatorname{PIP}(\mathbb{O})$ ). Since $d \not c$ and $a$ and $c$ satisfy (\$), we obtain that $a \not d \not c$, contradicting that $a$ and $c$ do not satisfy $(\boldsymbol{C})$

As we obtain a contradiction in all cases, we conclude that $\triangleleft_{\mathrm{S}} \subseteq \triangleleft$.

\section{A.4. Proof of Claims of Section 2.3.5.}

Proof of Claim 2.59. First, $\triangleleft^{\mathrm{PIP}^{ \pm} \mathrm{id}(\mathbb{O})}$ is clearly antisymmetric as it is contained in the antisymmetric relation $\triangleleft$. To prove that it is transitive, con-

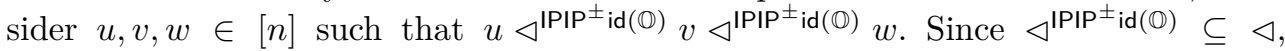
we have $u \triangleleft v \triangleleft w$, so that $u \triangleleft w$ by transitivity of $\triangleleft$. Assume by means of contradiction that $u \Varangle^{\mathrm{IPIP}^{ \pm} \mathrm{id}(\mathbb{O})} w$. Thus, $u<w$ and there exists $u \leqslant n<p \leqslant w$ such that $n \in\{u\} \cup \mathbb{O}^{-}$while $p \in\{w\} \cup \mathbb{O}^{+}$and $n \Varangle p$. We now distinguish three cases: 
- If $v \leqslant n$, then $n \nless p$ and $v \leqslant n<p \leqslant w$ contradicts our assumption that $v \triangleleft^{\mathrm{IPIP}^{ \pm}} \mathrm{id}(\mathbb{O}) w$.

- If $p \leqslant v$, then $n \Varangle p$ and $u \leqslant n<p \leqslant v$ contradicts our assumption that $u \triangleleft^{\mathrm{IPIP}^{ \pm}} \mathrm{id}(\mathbb{O}) v$.

- Finally, if $n<v<p$, then $u \triangleleft^{\mathrm{IPIP}^{ \pm} \mathrm{id}(\mathbb{O})} v$ ensures that $n \triangleleft v$, and $v \triangleleft^{\operatorname{IPIP}}{ }^{ \pm} \mathrm{id}(\mathbb{O})$ $w$ ensures that $v \triangleleft p$. Together with $n \Varangle v$, this contradicts the transitivity of $\triangleleft$.

As we obtained a contradiction in each case, we conclude that $\triangleleft^{\mathrm{PIP}^{ \pm}} \mathrm{id}(\mathbb{O})$ is transitive. Since all our relations are reflexive, we conclude that $\triangleleft^{I P^{ \pm}{ }^{ \pm}} \mathrm{id}(\mathbb{O})$ is a poset.

Proof of Claim 2.60. We prove that $\triangleleft^{\mathrm{IPIP}^{ \pm} \mathrm{id}(\mathbb{O})}$ is in $\operatorname{IPIP}^{+}(\mathbb{O})$, the result follows by symmetry for $\operatorname{IPIP}^{-}(\mathbb{O})$ and finally for $\operatorname{IPIP}^{ \pm}(\mathbb{O})=\operatorname{IPIP}^{+}(\mathbb{O}) \cap \operatorname{IPIP}^{-}(\mathbb{O})$. Assume that there exists $a<b<c$ with $b \in \mathbb{O}^{+}$and $a \Varangle^{\operatorname{IPIP}^{ \pm} \mathrm{id}(\mathbb{O})} b$. Then there are witnesses $a \leqslant n<p \leqslant b$ with $n \in\{a\} \cup \mathbb{O}^{-}$while $p \in\{b\} \cup \mathbb{O}^{+}$and $n \not p$. Since $b \in \mathbb{O}^{+}$, we have $p \in \mathbb{O}^{+}$. Therefore, $n$ and $p$ are also witnesses for $a \Varangle^{\operatorname{IPIP}^{ \pm} \text {id(O) }} c$. This shows that $\triangleleft^{\mathrm{PIP}^{ \pm}} \mathrm{id}(\mathbb{O})$ is in $\operatorname{IPIP}^{+}(\mathbb{O})$.

Proof of Claim 2.61. Consider $\varangle \in \operatorname{IWOIP}(n)$. Let $a<b<c$ in $[n]$ be such that

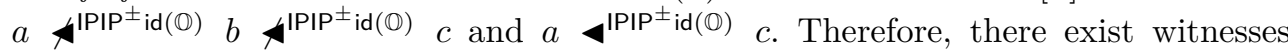
$a \leqslant m<p \leqslant b \leqslant n<q \leqslant c$ with $m \in\{a\} \cup \mathbb{O}^{-}, p \in\{b\} \cup \mathbb{O}^{+}, n \in\{b\} \cup \mathbb{O}^{-}$ and $q \in\{c\} \cup \mathbb{O}^{+}$, and such that $m \nless p$ and $n \nless q$. If $p \neq b$, then $p \in \mathbb{O}^{+}$ and $a \leqslant m<p<c$ are also witnesses for $a \lambda^{\operatorname{IPIP}^{ \pm}} \mathrm{id}(\mathbb{O}) c$. By symmetry, we can thus assume that $p=b=n$. Therefore, we have $m \boldsymbol{\wedge}=b=n \nless q$, which implies that $m$ since $\triangleleft \in \operatorname{IWOIP}(n)$. Since $a \leqslant m<q \leqslant c$ with $m \in\{a\} \cup \mathbb{O}^{-}, q \in\{c\} \cup \mathbb{O}^{+}$and $m$ we obtain that $a \wedge^{\mathrm{IPIP}^{ \pm} \mathrm{id}(\mathbb{O})} c$. We conclude that $\boldsymbol{\iota} \in \operatorname{IWOIP}(n)$ implies $\triangleleft^{\mathrm{IPIP}^{ \pm} \mathrm{id}(\mathbb{O})} \in$ $\operatorname{IWOIP}(n)$. In particular, we obtain that $\triangleleft^{\operatorname{IPIPid}(\mathbb{O})}=\left(\triangleleft^{\operatorname{IWOIPid}}\right)^{\operatorname{IPIP}{ }^{ \pm}} \mathrm{id}(\mathbb{O}) \in \operatorname{IPIP}(\mathbb{O})$ since $\triangleleft \operatorname{IWOIPid} \in \operatorname{IWOIP}(n)$ by Lemma 2.16 .

Proof of Claim 2.63. Claim 2.63 is immediate for $\varepsilon \in\{-,+\}$. For $\varepsilon= \pm$, assume that there exists $a \leqslant n<p \leqslant c$ with $n \in\{c\} \cup \mathbb{O}^{-}$and $p \in\{b\} \cup \mathbb{O}^{+}$such that $n \Varangle p$. Then we also have $n \nless p$ which implies $n \backslash c$ (since $\downarrow \in \operatorname{IPIP}^{+}(\mathbb{O})$ ) and $a$ ( since $\triangleleft \in \mathrm{IPIP}^{-}(\mathbb{O})$ ), which in turn implies $a \wedge c$. Finally, this also implies the claim when $\varepsilon=\varnothing$ by applying first Lemma 2.18 and then the claim for $\varepsilon= \pm$.

Proof of Claim 2.65. Otherwise, there exists $a<c$ such that there is no $\mathbb{O}$-snake from $a$ to $c$ in $\varangle$. Choose such a pair $a<c$ with $c-a$ minimal. Since $\triangleleft_{\sigma}$ is a total order, we have either $a \triangleleft_{\sigma} c$ or $a \triangleright_{\sigma} c$. Assume for example that $a \triangleleft_{\sigma} c$, the other case being symmetric. Since $a \triangleleft_{\sigma} c$ while $a>c$ (otherwise $a \triangleleft c$ is an $\mathbb{O}$-snake), there exists $a \leqslant n<p \leqslant c$ such that $n \in\{a\} \cup \mathbb{O}^{-}$while $p \in\{c\} \cup \mathbb{O}^{+}$and $n \not_{\sigma} p$. Since $\triangleleft_{\sigma}$ is a total order, we get $n \triangleright_{\sigma} p$. Moreover, we have either $a \triangleleft_{\sigma} n$ or $a \triangleright_{\sigma} n$. In the latter case, we get by transitivity of $\triangleleft_{\sigma}$ that $a \triangleright_{\sigma} p$. Therefore, up to forcing $a=n$, we can assume that $a \triangleleft_{\sigma} n$ and similarly up to forcing $p=c$, we can assume that $p \triangleleft_{\sigma} c$. It follows that $a \triangleleft_{\sigma} n \triangleright_{\sigma} p \triangleleft_{\sigma} c$ is an $\mathbb{O}$-snake from $a$ to $c$ in $\triangleleft_{\sigma}$, where either $a \neq n$ or $p \neq c$ (because $a \triangleleft_{\sigma} c$ ). By minimality of $c-a$ in our choice of $a<c$, there exists an $\mathbb{O}$-snake from $a$ to $n$, from $n$ to $p$, and from $p$ to $c$ in ४. Since $n \in\{a\} \cup \mathbb{O}^{-}$ and $p \in\{c\} \cup \mathbb{O}^{+}$, it is straightforward to construct from these snakes an $\mathbb{O}$-snake from $a$ to $c$ in $\varangle$, contradicting our assumption.

Proof of Claim 2.69. Assume that there exists $a<c$ incomparable in $\varangle$ that do not satisfy (\$) in 4 . We choose such a pair $a<c$ with $c-a$ minimal. By symmetry, we can assume that there exists $a<b<c$ such that $a \triangleleft b \vec{b}$ and that $b$ is maximal for this property. Since $\triangleleft \subseteq \triangleleft$, we have $a \triangleleft b$. We distinguish three cases: 
(1) Assume first that $a$ and $c$ are incomparable in $\triangleleft$. Since $\triangleleft \in \operatorname{WOFP}(n)$, Proposition 2.10 and $a \triangleleft b$ imply that $a \triangleleft b \triangleright c$. Since $b \triangleright c$ while $b \triangleleft c$, there is $b \leqslant p<n \leqslant c$ with $p \in\{b\} \cup \mathbb{O}^{+}$while $n \in\{c\} \cup \mathbb{O}^{-}$and $p \not n$. We again have two cases:

- If $b \neq p$, we have $p \not c$ (since otherwise $p \not n$ would contradict that $\varangle \in \operatorname{DPIP}(\mathbb{O})$ ) and thus $a \not p$ (by maximality of $b$ ). We thus obtained $a<p<c$ with $p \in \mathbb{O}^{+}$and $a<p$, so that $a<c$ satisfy in 4 .

- If $b=p$, then $n \neq c$. We have $a \not n$ (since otherwise $p \not n$ would contradict that $\varangle \in \operatorname{PIP}(\mathbb{O})$ ). Moreover, by minimality of $c-a$, we have $b=p$ and $c$ satisfy $(\mathbf{s})$ in $\boldsymbol{\downarrow}$, so that $p$ implies that $n<c$. We obtained that $a<n<c$ with $n \in \mathbb{O}^{-}$and $a \ngtr n \nless c$, so that $a<c$ satisfy in 4 .

(2) Assume now that $a \triangleleft c$. Since $a \triangleleft c$ while $a \ngtr c$, there is $a \leqslant n<p \leqslant c$ with $n \in\{a\} \cup \mathbb{O}^{-}$while $p \in\{c\} \cup \mathbb{O}^{+}$and $n \Varangle p$. Since $\triangleleft \in \operatorname{PIP}(\mathbb{O})$ and $n \nless p$, we must have $a \nless p$ and $n \nless c$. Assume that $a$ and $c$ do not satisfy ( $)$ in 4 . This implies that $p \triangleright c$ and $a \triangleright n$. Since $a \downarrow c$ and $p \triangleright c$, we obtain by transitivity of $\varangle$ that $a$ and $p$ are incomparable in $\varangle$. By minimality of $c-a$, we obtain that $a$ and $p$ satisfy $(\boldsymbol{\beta})$. We now consider two cases:

- If $b<p$, then $a<b$ implies that $b>p$, which together with $p>c$ and $b \downarrow c$ contradicts the transitivity of 4 .

- If $p \leqslant b$, then we have $a \leqslant n<p \leqslant b$ with $n \in\{a\} \cup \mathbb{O}^{-}$while $p \in\{c\} \cup \mathbb{O}^{+}$ and $n \nless p$, which contradicts that $a \triangleleft b$.

Since we obtained a contradiction in both cases, we conclude that $a$ and $c$ satisfy $(\mathbf{\infty})$ in 4 .

(3) Assume finally that $a \triangleright c$. Then $a \not b$ and $\triangleleft \in \operatorname{DWOIP}(n)$ implies that $a \triangleleft b \triangleright c$ and we are back to case (1).

\section{A.5. Proof of Claims of Theorem 2.77.}

Proof of Claim 2.78. As $\mathrm{cf}_{\mathrm{DPIP}(\mathbb{O})}$ is decreasing, we only consider $(a, c) \in \dashv^{\text {Dec }}$. Since $\dashv^{\text {Dec }}=\triangleleft^{\text {Dec }} \cap \varangle^{\text {Dec }}$, we have $a \triangleright c$ and $a \triangleright c$. Since both $\triangleleft, \varangle \in \operatorname{DPIP}(\mathbb{O})$, for any $a<b<c$, if $b \in \mathbb{O}^{-}$then $a \triangleright b$ and $a \triangleright b$ so that $a \vdash b$, while if $b \in \mathbb{O}^{+}$ then $b \triangleright c$ and $b \triangleright c$ so that $b \vdash c$. Note that the important point here is that the behavior of $b$ is the same in $\triangleleft$ and $\triangleleft$ as it is dictated by the orientation of $b$.

Proof of Claim 2.79. Assume that $\dashv^{\text {tdd }} \notin \operatorname{DPIP}(\mathbb{O})$. Consider $\{a, c\} \in \operatorname{cf}_{\operatorname{DPIP}(\mathbb{O})}\left(\dashv^{\text {tdd }}\right)$ with $a<c$ and $c-a$ minimal. Since $\operatorname{cf}_{\operatorname{DPIP}(\mathbb{O})}\left(\dashv^{\text {tdd }}\right)$ is decreasing, we have $a \vdash^{\text {tdd }} c$. Assume for the moment that there exists $a<b<c$ such that $b \in \mathbb{O}^{-}$and $a \nvdash^{\text {tdd }} b$, and choose such $b$ with $b-a$ minimal. Since $\dashv \in \operatorname{DPIP}(\mathbb{O})$, we have $a \vdash b$ while $a \nvdash^{\text {tdd }} b$. By definition of $\dashv^{\text {tdd }}$, there exists $i \leqslant b$ and $j \geqslant a$ such that $i \dashv b \dashv a \dashv j$ but $i \dashv j$. From Lemma 1.15, we know that either $i \neq b$ or $j \neq a$. We thus distinguish two cases.

(1) Assume that $i \neq b$. Again by Lemma 1.15, there exists $a<k<b$ such that $i \dashv k \dashv b$. Thus, we have $k \nvdash b$ (since $\dashv$ is antisymmetric) while $a \vdash b$

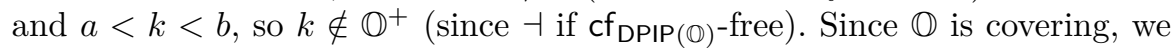
therefore obtain that $k \in \mathbb{O}^{-}$. By minimality of $b-a$ in our choice of $b$, we obtain that $a \vdash^{\text {tdd }} k$. But $i \dashv k \dashv a \dashv j$ and $a \vdash^{\text {tdd }} k$ implies that $i \dashv j$, a contradiction to our assumption on $i$ and $j$.

(2) Assume now that $j \neq a$. Again by Lemma 1.15, there exists $a<k<b$ such that $a \dashv k \dashv j$. Thus, we have $a \nvdash k$ (since $\vdash$ is antisymmetric) while $a \vdash b$

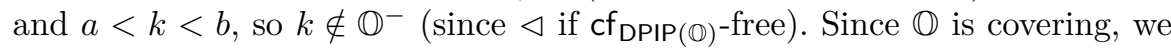
therefore obtain that $k \in \mathbb{O}^{+}$. Since $\dashv \in \operatorname{DPIP}(\mathbb{O}), k \in \mathbb{O}^{+}$and $a \vdash c$, we 
have $k \vdash c$. We claim that $k \vdash^{\text {tdd }} c$. Otherwise we could find $i^{\prime} \leqslant c$ and $j^{\prime} \geqslant k$ such that $i^{\prime} \dashv c \dashv k \dashv j^{\prime}$ while $i^{\prime} \nrightarrow j^{\prime}$. Since $a \dashv k \dashv j^{\prime}$ and $\dashv$ is semitransitive, we would also have $i^{\prime} \dashv c \dashv a \dashv j^{\prime}$ while $i^{\prime} \dashv j^{\prime}$, contradicting the fact that $a \vdash^{\text {tdd }} c$. Now by minimality of $c-a$ in our choice of $(a, c)$, we obtain that $(c, k) \in \dashv^{\text {tdd }} \backslash \mathrm{cf}_{\operatorname{DPIP}(\mathbb{O})}\left(\dashv^{\text {tdd }}\right)$. Therefore, since $b \in \mathbb{O}^{-}$, we have $k \vdash^{\text {tdd }} b$. But $i \dashv b \dashv k \dashv j$ and $k \vdash^{\text {tdd }} b$ implies that $i \dashv j$, a contradiction to our assumption on $i$ and $j$.

We therefore proved that $a \vdash^{\text {tdd }} b$ for all $a<b<c$ with $b \in \mathbb{O}^{-}$. The case of $b \in \mathbb{O}^{+}$ is symmetric and left to the reader. This concludes the proof.

\section{A.6. Proof of Claims of Proposition 2.89.}

Proof of Claim 2.90. Assume that $\dashv$ is not $\mathrm{cf}_{\mathrm{sn}(\mathbb{O})}$-free and let $\{a, c\} \in \mathrm{cf}_{\mathrm{sn}(\mathbb{O})}(\dashv)$ with $a<c$ and $c-a$ minimal. Since $a \nrightarrow c$, we have $a \not c$ and $a \nmid c$. Since $a \nvdash c$, we have $a \not c$ or $a$. We can thus assume without loss of generality that $a$ and $c$ are incomparable in $\triangleleft$. Since $\triangleleft$ is $\mathrm{cf}_{\mathrm{sn}(\mathbb{O})}$-free, there exists $a<b_{1}<\cdots<b_{k}<c$ such that either $b_{2 i} \in \mathbb{O}^{+}, b_{2 i+1} \in \mathbb{O}^{-}$and $a \triangleleft b_{1} \triangleright b_{2} \triangleleft b_{3} \triangleright \cdots$, or $b_{2 i} \in \mathbb{O}^{-}, b_{2 i+1} \in \mathbb{O}^{+}$ and $a \triangleright b_{1} \triangleleft b_{2} \triangleright b_{3} \triangleleft \cdots$. We distinguish these two cases:

(1) In the former case, we obtain $b_{1} \in \mathbb{O}^{-}$and $a \dashv b_{1}$ ( since $\triangleleft^{\operatorname{lnc}} \subseteq \dashv$ ).

(2) In the latter case, we distinguish three cases according to the order of $a$ and $b_{1}$ in 4

- if $a \triangleleft b_{1}$, then $a \dashv b_{1} \dashv b_{2}$ (since $\triangleleft^{\operatorname{lnc}} \cup \triangleleft^{\operatorname{lnc}} \subseteq \dashv$ ) so that we obtain $b_{2} \in \mathbb{O}^{-}$and $a \dashv b_{2}$.

- if $a \triangleright b_{1}$, we obtain $b_{1} \in \mathbb{O}^{+}$and $a \vdash b_{1}\left(\right.$ since $\left.\triangleleft^{\text {Dec }} \cap \triangleleft^{\text {Dec }} \subseteq \dashv\right)$.

- if $a$ and $b_{1}$ are incomparable in $\varangle$, then they are also incomparable in $\dashv$. By minimality of $c-a$ in our choice of $(a, c)$, we have $\left\{a, b_{1}\right\} \notin \mathrm{cf}_{\mathrm{sn}(\mathbb{O})}(-1)$. Lemma $2.47(3)$ thus ensures the existence of $a<b<b_{1}$ such that either $b \in \mathbb{O}^{-}$and $a \dashv b$, or $b \in \mathbb{O}^{+}$and $a \vdash b$.

In all situations, we have found $a<b<c$ such that either $b \in \mathbb{O}^{-}$and $a \dashv b$, or $b \in \mathbb{O}^{+}$and $a \vdash b$. Since $\{b, c\} \notin \mathrm{cf}_{\mathrm{sn}(\mathbb{O})}(\dashv)$ by minimality of $c-a$ in our choice of $(a, c)$, Lemma $2.47(3)$ thus contradicts that $\{a, c\} \in \mathrm{cf}_{\mathrm{sn}(\mathbb{O})}(\dashv)$.

Proof of Claim 2.91. Assume that $\dashv^{\text {tdd }}$ is not $\mathrm{cf}_{\mathrm{sn}(\mathbb{O})}$-free and let $\{a, c\} \in \mathrm{cf}_{\mathrm{sn}(\mathbb{O})}\left(\dashv^{\text {tdd }}\right)$ with $a<c$ and $c-a$ minimal. We distinguish two cases:

(1) If $a \nvdash c$, since $\dashv$ is $\mathrm{cf}_{\mathrm{sn}(\mathbb{O})}$-free, Lemma $2.47(3)$ ensures that there exists $a<b<c$ such that $b \in \mathbb{O}^{-}$and $a \dashv b$, or $b \in \mathbb{O}^{+}$and $a \vdash b$. In the former case, we also have $a \dashv^{\text {tdd }} b$. In the latter case, we either have $a \vdash^{\text {tdd }} b$ or $a$ and $b$ are incomparable in $\dashv^{\text {tdd }}$. By minimality of $c-a$ in our choice of $(a, c)$, we have $\{a, b\} \notin \mathrm{cf}_{\mathrm{sn}(\mathbb{O})}\left(\dashv^{\text {tdd }}\right)$. We thus obtain by Lemma $2.47(3)$ that there

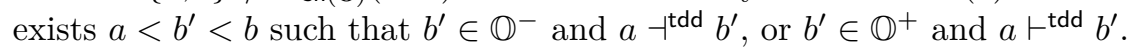

(2) If $a \vdash c$, then there exists $i \leqslant c$ and $j \geqslant a$ such that $i \dashv c \dashv a \dashv j$ while $i \dashv j$. From Lemma 1.15, we can assume for example that $i \neq c$ so that there exists $a<k<c$ with $i \dashv k \dashv c$ (the proof when $j \neq a$ is similar). Note that $a f^{\text {tdd }} k$ since otherwise $a \dashv^{\text {tdd }} k-^{\text {tdd }} c$ and $a \AA^{\text {tdd }} c$ would contradict the transitivity of $\dashv^{\text {tdd }}$. Moreover, $a \nvdash^{\text {tdd }} k$ since either we already have $a \not k k$, or $i \leqslant c$ and $j \geqslant a$ still satisfy $i \dashv k \dashv a \dashv j$ while $i \rtimes j$. Therefore, $a$ and $k$ are incomparable in $\dashv$. By minimality of $c-a$ in our choice of $(a, c)$, we have $\{a, k\} \notin \mathrm{cf}_{\mathrm{sn}(\mathbb{O})}\left(\dashv^{\text {tdd }}\right)$. We thus obtain by Lemma $2.47(3)$ that there exists $a<b<k$ such that $b \in \mathbb{O}^{-}$and $a-^{\text {tdd }} b$, or $b \in \mathbb{O}^{+}$and $a \vdash^{\text {tdd }} b$. 
In all situations, we have found $a<b<c$ such that either $b \in \mathbb{O}^{-}$and $a$ - $^{\text {tdd }} b$, or $b \in \mathbb{O}^{+}$and $a \vdash^{\text {tdd }} b$. Since $\{b, c\} \notin \mathrm{cf}_{\mathrm{sn}(\mathbb{O})}\left(\dashv^{\text {tdd }}\right)$ by minimality of $c-a$ in our choice of $(a, c)$, Lemma $2.47(3)$ thus contradicts that $\{a, c\} \in \mathrm{cf}_{\mathrm{sn}(\mathbb{O})}\left(\dashv^{\mathrm{tdd}}\right)$.

Acknowledgements. The computation and tests needed along the research were done using the open-source mathematical software SAGE [19] and its combinatorics features developed by the SAGE-COMBINAT community [18].

\section{REFERENCES}

[1] Anders Björner and Michelle L. Wachs, Permutation statistics and linear extensions of posets, J. Comb. Theory, Ser. A 58 (1991), no. 1, 85-114.

[2] Grégory Chatel and Vincent Pilaud, Cambrian Hopf Algebras, Adv. Math. 311 (2017), 598-633.

[3] Grégory Châtel and Viviane Pons, Counting smaller elements in the Tamari and $m$-Tamari lattices, J. Comb. Theory, Ser. A 134 (2015), 58-97.

[4] Aram Dermenjian, Christophe Hohlweg, and Vincent Pilaud, The facial weak order and its lattice quotients, Trans. Am. Math. Soc. 370 (2018), no. 2, 1469-1507.

[5] Christophe Hohlweg and Carsten Lange, Realizations of the associahedron and cyclohedron, Discrete Comput. Geom. 37 (2007), no. 4, 517-543.

[6] Christian Kassel, Alain Lascoux, and Christophe Reutenauer, The singular locus of a Schubert variety, J. Algebra 269 (2003), no. 1, 74-108.

[7] Daniel Krob, Matthieu Latapy, Jean-Christophe Novelli, Ha-Duong Phan, and Sylviane Schwer, Pseudo-Permutations I: First Combinatorial and Lattice Properties, 13th International Conference on Formal Power Series and Algebraic Combinatorics (FPSAC 2001), 2001, http: //www-igm.univ-mlv.fr/ novelli/ARTICLES/pp1.ps.

[8] Carsten Lange and Vincent Pilaud, Associahedra via spines, Combinatorica 38 (2018), no. 2, $443-486$.

[9] Jean-Louis Loday, Realization of the Stasheff polytope, Arch. Math. 83 (2004), no. 3, 267-278.

[10] Folkert Müller-Hoissen, Jean Marcel Pallo, and Jim Stasheff (eds.), Associahedra, Tamari Lattices and Related Structures. Tamari Memorial Festschrift, Progress in Mathematics, vol. 299, Birkhäuser, 2012.

[11] Jean-Christophe Novelli and Jean-Yves Thibon, Polynomial realizations of some trialgebras, 18th International Conference on Formal Power Series and Algebraic Combinatorics (FPSAC 2006), https://arxiv.org/abs/math/0605061, 2006.

[12] Patricia Palacios and María O. Ronco, Weak Bruhat order on the set of faces of the permutohedron and the associahedron, J. Algebra 299 (2006), no. 2, 648-678.

[13] Vincent Pilaud and Viviane Pons, IntegerPosets, 2018, Research code. https://github.com/ VivianePons/public-notebooks/tree/master/IntegerPosets.

[14] , Permutrees, Algebr. Comb. 1 (2018), no. 2, 173-224.

[15] Nathan Reading, Lattice congruences of the weak order, Order 21 (2004), no. 4, 315-344.

[16] _ Cambrian lattices, Adv. Math. 205 (2006), no. 2, 313-353.

[17] N. J. A. Sloane, The On-Line Encyclopedia of Integer Sequences, 2010, http://oeis.org.

[18] The Sage-Combinat community, Sage-Combinat: enhancing Sage as a toolbox for computer exploration in algebraic combinatorics, 2016, http://wiki.sagemath.org/combinat.

[19] The Sage Developers, SageMath, the Sage Mathematics Software System, 2016, http://www. sagemath.org/.

Grégory Chatel, LIGM, Univ. Paris-Est Marne-la-Vallée, 5 Boulevard Descartes, 77420 Champssur-Marne, France

E-mail : gregory.chatel@univ-paris-est.fr

Url : http://igm.univ-mlv.fr/ gchatel/

Vincent Pilaud, CNRS \& LIX, École Polytechnique, 1 Rue Honoré d'Estienne d'Orves, 91120 Palaiseau, France

E-mail : vincent.pilaud@lix.polytechnique.fr

Url : http://www.lix.polytechnique.fr/ pilaud/

Viviane Pons, LRI, Univ. Paris-Sud, Bâtiment 650, Rue Noetzlin, 91190 Gif-sur-Yvette, France E-mail : viviane.pons@lri.fr Url : http://www.lri.fr/ pons/ 